University of Louisville ThinkIR: The University of Louisville's Institutional Repository

Electronic Theses and Dissertations

$5-2016$

\title{
Influence of in-office oral hygiene instructions on the parental assessment of their child's oral health.
}

Sireesha Kodali

University of Louisville

Follow this and additional works at: https://ir.library.louisville.edu/etd

Part of the Dental Public Health and Education Commons, and the Pediatric Dentistry and Pedodontics Commons

\section{Recommended Citation}

Kodali, Sireesha, "Influence of in-office oral hygiene instructions on the parental assessment of their child's oral health." (2016). Electronic Theses and Dissertations. Paper 2440.

https://doi.org/10.18297/etd/2440

This Master's Thesis is brought to you for free and open access by ThinkIR: The University of Louisville's Institutional Repository. It has been accepted for inclusion in Electronic Theses and Dissertations by an authorized administrator of ThinkIR: The University of Louisville's Institutional Repository. This title appears here courtesy of the author, who has retained all other copyrights. For more information, please contact thinkir@louisville.edu. 


\title{
INFLUENCE OF IN-OFFICE ORAL HYGIENE INSTRUCTIONS ON THE PARENTAL ASSESSMENT OF THEIR CHILD'S ORAL HEALTH
}

\author{
By, \\ Sireesha Kodali, MHA, M.S, DMD \\ University of Louisville, 2016
}

\begin{abstract}
A Thesis
Master of Science

Oral Biology

University of Louisville

Louisville, Kentucky
\end{abstract}

Submitted to the Faculty of the School of Dentistry of the University of Louisville In Partial Fulfillment of the Requirements for the Degree of

May, 2016 

INFLUENCE OF IN-OFFICE ORAL HYGIENE INSTRUCTIONS ON PARENTAL ASSESSMENT OF THEIR CHILD'S ORAL HEALTH

\author{
By \\ Sireesha Kodali \\ M.S, University of Louisville, 2016 \\ A Thesis Approved on
}

April 06, 2016

By the following Thesis Committee:

Dr. Ann Lake Greenwell, Thesis Mentor

Dr. Douglas Darling, Committee Member

Dr. Lee Mayer, Committee Member 


\section{DEDICATION}

This thesis is dedicated to my friends and family members for their love and support. Without their encouragement, this thesis would not have been possible. 


\section{ACKNOWLEDGEMENTS}

I would like to thank my thesis mentor, Dr. Ann Lake Greenwell, for providing me an invaluable opportunity to work under her guidance, and for extending her continued guidance and support to this research project with her immense expertise in the specialty of pediatric dentistry.

I would also like to thank Dr. Douglas Darling, Thesis Committee member for his constant support and enthusiasm in all phases of the research. It was his relentless mentorship that made this project a successful endeavor.

I would also like to express my thanks to Dr. Lee Mayer, Thesis Committee member, for his help to refine this project with his knowledge.

I also thank Dr. Bakeerathan Gunaratnam for extending his guidance with statistical analysis. 


\section{ABSTRACT \\ INFLUENCE OF IN-OFFICE ORAL HYGIENE INSTRUCTIONS ON PARENTAL ASSESSMENT OF THEIR CHILD'S ORAL HEALTH \\ Sireesha Kodali}

April 06, 2016

Purpose: To identify the relation between oral health education and parental assessment of their child's dental condition.

Methods: Our study population included 33,342 children, from Smile KY! annual screenings from 2002 to 20014. Data collection was through questionnaire forms. Our primary risk factor was "dental health instructions received" (yes/no) and our outcome of interest was "parental assessment of your child's dental health" (good/fair/poor). We had 13 years of data from 2002 to 2014 and used 'SAS' statistical software for data manipulation and recoding. Descriptive statistics, chi-square test of independence, trend analysis and multiple logistic regression modeling were performed on 'SPSS' and 'SAS' statistical software at $P<0.05$.

Results: While $17.6 \%$ of our population had no prior dental education training, more than $80 \%$ of those receiving instructions were given by different providers: $66.7 \%$ dental office, $1.1 \%$ health department, $1.8 \%$ physician office, $2.4 \%$ other sources and the rest from a multiple of providers. Seventy-three percent of parents rated their child's dental health as good and $26.7 \%$ assessed it as fair/poor. There was a correlation between the parental 
assessments and oral health training. We found that dental office education played a key role in influencing parental assessments with a $P<0.0001$.

Conclusions: Comparing the effects of oral health education given in dental offices, physician offices, health departments and other providers; showed that education provided in dental offices had significant influence on the parent's perception of good oral health for their child.

Key words: Parental assessment of oral health, oral health education, physician office oral health education, dental office oral health education, health department oral health education 
TABLE OF CONTENTS

ACKNOWLEDGEMENTS




\section{LIST OF FIGURES}

FIGURE $\quad$ PAGE

Figure 1: Frequency of Parental Assessment ........................................................ 15

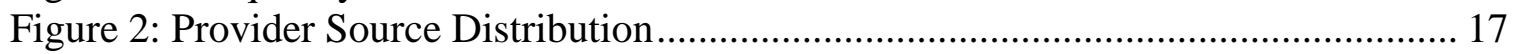

Figure 3: Relation Between Parental Assessment and Oral Health Education................. 18

Figure 4: Relation Between Oral Health Education and Parental Assessments in Bullitt

County (subset with multiple dental visits) ......................................................... 19

Figure 5: Relation Between Oral Health Education and Parental Assessments in Jefferson

County (subset with unique dental visits) ............................................................ 20

Figure 6: Relation Between Parental Assessment and Oral Health Education from Dental

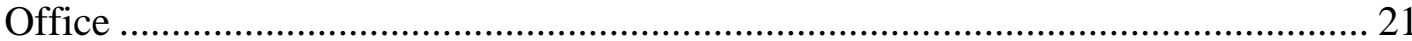

Figure 7: Relation Between Parental Assessment and Oral Health Education from

Physician Office..................................................................................... 22

Figure 8: Relation Between Parental Assessment and Oral Health Education from Health

Department.................................................................................................... 23

Figure 9: Relation Between Parental Assessment and Oral Health Education from 'Other'

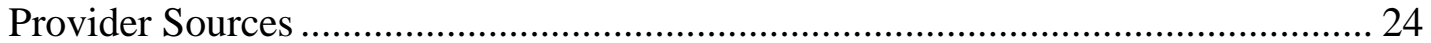

Figure 10: Relation Between Clinical Screening and Oral Health Education .................. 25

Figure 11: Relation Between Clinical Screening and Oral Health Education from Dental

Office for Bullitt County (Subset with repeated dental visits) .............................. 26

Figure 12: Relation Between Clinical Screening and Oral Health Education for Jefferson County (Subset with unique dental visits) .................................................... 27

Figure 13: Relation Between Clinical Screening and Oral Health Education from a Dental

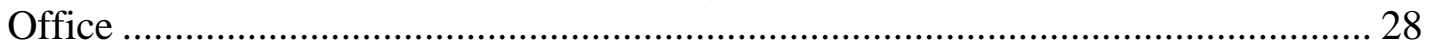

Figure 14: Relation Between Clinical Screening and Oral Health Education from a

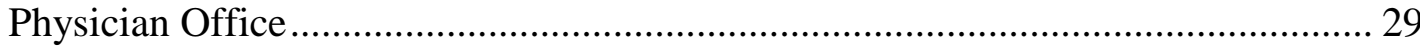

Figure 15: Relation Between Clinical Screening and Oral Health Education from a Health

Department......................................................................................... 30

Figure 16: Relation Between Clinical Screening and Oral Health Education from 'Other'

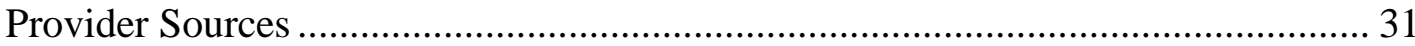

Figure 17: Comparison of Parental Assessment with Clinical Screening Results........... 32

Figure 18: Comparison of Parental Assessment with Clinician Screening Results Based

on Oral Health Training from Different Providers - Total Correct Percentage ........ 35

Figure 19: Comparison of Parental Assessment with Clinician Screening Results Based

on Oral Health Training from Different Providers - Total Wrong Percentage ......... 36

Figure 20: Representation of Odds Ratio for 'Fair' Parental Assessments ...................... 39

Figure 21: Representation of Odds Ratio for 'Poor' Parental Assessments .................... 40 
Figure 22: Trend Analysis for Carious Lesions Over 11 years of Screening .................. 41 Figure 23: Trend Analysis for Dental Office Visits Over the 11 Years of Smile KY!.... 42 Figure 24: Trend Analysis for Dental Insurance Over the 11 Years History of Smile KY! 


\section{LIST OF TABLES}

$\begin{array}{ll}\text { TABLE } & \text { PAGE }\end{array}$

Table 1: Descriptive characteristics of Study Population ............................................. 13

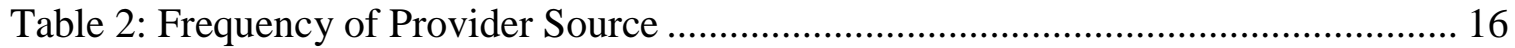

Table 3: Chi-square Test of Independence Between Dental Health Education and Parental

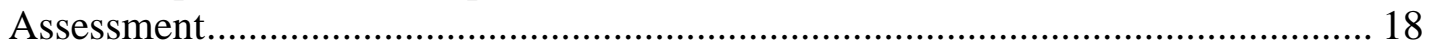

Table 4: Chi-square Test Between Dental Health Training and Parental Assessment -

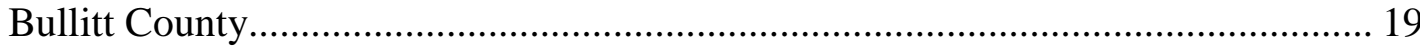

Table 5: Chi-square Test Between Dental Health Training and Parental Assessment Jefferson County ............................................................................................ 20

Table 6: Chi-square Test Between Dental Office Instructions and Parental Assessment 21 Table 7: Chi-square Test Between Physician Office Instructions and Parental Assessment

Table 8: Chi-square Test Between Health Department and Parental Assessment........... 23

Table 9: Chi-square Test Between 'Other' Provider Sources and Parental Assessment.. 24

Table 10: Chi-square Test Between Dental Health Training and Clinical Screening ...... 25

Table 11: Chi-square Test Between Dental Health Training and Clinical Screening -

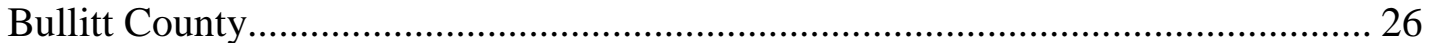

Table 12: Chi-square Test Between Dental Health Training and Clinical Screening Jefferson County ....................................................................................... 27

Table 13: Chi-square Test Between Dental Office Instructions and Clinical Screening.. 28

Table 14: Chi-square Test Between Physician Office Instructions and Clinical Screening

Table 15: Chi-square Test Between Health Department Instructions and Clinical Screening.

Table 16: Chi-square Test Between 'Other' Provider Instructions and Clinical Screening

Table 17: Chi-square Test Between Parental Assessment and Clinical Screening .......... 32

Table 18: Chi-square Test Between Parental Assessment Based on Instructions from a Dental Office and Clinical Screening Results .................................................... 33

Table 19: Chi-square Test Between Parental Assessment Based on Instructions from a Physician Office and Clinical Screening Results.................................................. 33

Table 20: Chi-square Test Between Parental Assessment Based on Instructions from a Health Department and Clinical Screening Results .......................................... 33

Table 21: Chi-square Test Between Parental Assessment Based on Instructions from Other Providers and Clinical Screening Results ................................................... 34

Table 22: Chi-square Test Between Parental Assessment Based on 'NO’ Instructions Received from Any of the Providers and Clinical Screening Results 34 
Table 23: Multivariate Logistic Regression Model (Base Level=0, Good): Good vs Fair

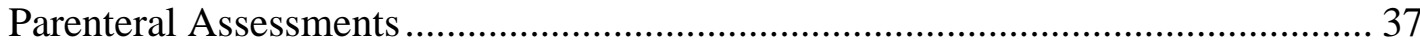

Table 24: Multivariate Logistic Regression Model (Base Level=0, Good): Good vs Poor

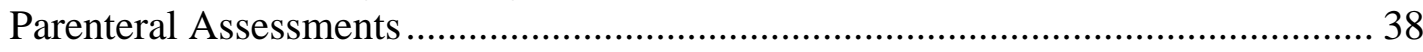

Table 25: Classification Table for Multiple Logistic Regression Modeling ................... 38 


\section{INTRODUCTION AND LITERATURE REVIEW}

The Centers for Disease Control and Prevention (CDC) ranked Kentucky second in poor dental health in the nation in $2008 .{ }^{1}$ Four years later in 2012, the ranking was improved to fifth position. ${ }^{2}$ According to a 2010 survey of Kentucky adults found that $61 \%$ of those aged 65 years and older were edentulous. ${ }^{3}$ As of 2015, only $61 \%$ of Kentucky adults reported having had a dental check-up within the past year. ${ }^{4}$ Childress et.al, found that among adults in Kentucky over 18 years of age, complete tooth loss was observed in $13 \%,{ }^{5}$ when compared to $6 \%$ in the entire US. This places Kentucky among the highest percentage of edentate states. There appears to be a significant dental crisis for adults in Kentucky who don't seem to have an understanding of the importance of maintaining a healthy dentition and how to accomplish a healthy dentition.

A survey of children in south central Kentucky $50 \%$ had untreated carious teeth. ${ }^{7}$ This evidence seems to demonstrate a significant need for widespread oral health education with emphasis on prevention for the youngest Kentuckians to prevent earliest forms of dental disease. ${ }^{5}$ In a 2001 Kentucky survey $17 \%$ of the parents rated their child's dental condition as fair/poor. ${ }^{8}$ Studies ${ }^{9-11}$ that have investigated the outcomes of poor oral health on children have found an increase in missed school days, poor academic performance, 
lowered self-esteem, inappropriate behavior and engagement in risky extra-curricular activities.

In Louisville, Kentucky and the eight surrounding counties a community service project has been operating since 2002 on the Give Kids A Smile template. This project is called "Smile KY!" and includes education, screening and free treatment in selected elementary schools. Approximately $33 \%$ of the children screened for Smile KY! had dental caries. ${ }^{6}$

The reasons behind poor oral health in early childhood can be multifactorial. Research has shown that parent attitudes and education play an important role their child's oral health. ${ }^{12,13}$ In addition to the vertical transmission of parental bacteria to the child's oral cavity, parental behaviors pertaining to oral health have been shown to influence their children's oral health determined by caries rate and the health of the gingiva. ${ }^{14}$ Studies have shown that a child's oral health is directly affected by maternal oral hygiene and can be improved by the mother's own brushing skills. ${ }^{15-19}$ Another study of 9 year-old children revealed that maternal oral health knowledge improved their child's oral health. ${ }^{20}$ External factors can influence parental ability to take care of their child's oral health, such as access to care, ${ }^{21,22}$ insurance coverage and income status. ${ }^{23-26}$ A study conducted in Saudi Arabia, found that there was a lack of adequate knowledge among Saudi parents concerning their child's oral wellbeing. ${ }^{27}$ Other studies have investigated the relationship between maternal knowledge, attitudes, ${ }^{28-30}$ level of anxiety, amount of formal education, ${ }^{31}$ behavioral and psychosocial factors and brushing practices ${ }^{32}$ in children of different age groups. Different age groups of children studied included preschool children ${ }^{12,33,34}, 2$ to 5 year old children ${ }^{17}$, 
5 to 9 year olds, 6 to $14 \mathrm{yr}$ olds and adolescents. ${ }^{35}$ Few studies have focused on children with special needs like autism or mental disabilities. ${ }^{19,36}$

A systemic review evaluating parental and child perceptions about oral health and how it affected their quality of life revealed that asking the appropriate questions can be helpful in obtaining information. ${ }^{37}$ Bhavana et.al developed the "Early Childhood Oral Health Impact Scale" to measure the oral health related quality of life among preschool children and their families. ${ }^{33}$ Studies related to parental oral health assessment are limited by incomplete questionnaires, which leaves the information obtained as less useful in determining associations. ${ }^{37,38}$ A study by Jokovic et.al found the most common limitation associated with a questionnaire to be recall bias leading to increased numbers of "don't knows" in a survey. ${ }^{38}$

Many studies have found a relationship between parental knowledge and their child's oral health status. Factors influencing parental knowledge are not always clear. The influence of a healthcare provider delivering instructions to a parent has not been tested. Parental perceptions play a vital role in a child's oral health. Intervention with counseling by a medical provider followed by referral to a dental provider showed great impact on reducing early childhood caries..$^{39,40} \mathrm{We}$ are interested in investigating the influence of different healthcare providers ${ }^{22,41}$ that parents and children have regular access to such as physician offices, health departments and dental offices.

The aim of this study was to identify the role played by various healthcare providers such as dentist, physician and health department personnel in influencing parental awareness and understanding of the importance of oral healthcare for their child. Our initial objective was to determine the amount of association between professionally delivered oral 
hygiene instructions and parental assessment of their child's oral health status. Then we wanted to specifically identify which provider's message carried the most influence on the parental assessment of the child's oral health status. Finally we wanted to identify other factors such as gender, ethnicity, previous dental visits and enrollment in health insurance that may have influenced the parental assessment of the child's oral health status. 


\section{METHODS}

\section{Data Collection}

Smile KY! is the Louisville, Kentucky version of Give Kids A Smile. It has been screening, educating and treating elementary age children for 12 years in Louisville and the eight surrounding counties. The data from these screenings have been complied in a database. Our study population included 34,689 children from the Smile KY! annual screenings which started in 2002 when the University of Louisville IRB approved the use of the database for research projects. The health history and the dental screening forms were the same forms used for statewide surveys conducted every few years. After collection, the data was entered into the database annually through 2014.

Every year 28 to 34 elementary schools in the eight county area were selected to participate in the Smile KY! community project. The elementary school staff gave our questionnaires to the children to carry home for the parents to fill out and return to the school. The questionnaires completed by the parents asked the following seven questions:

1. Does your child have insurance? - Medicaid/CHIP/Private Insurance/None

2. Please describe the condition of your child's teeth - Good/Fair/Poor

3. When was the last time your child visited a dentist?

- Within the past year

- One to three years ago

- More than three years ago

- Child has never seen a dentist

- Don't know

4. What was the main reason for your child's last visit to a dentist?

- Check-up, cleaning or exam

- Something was wrong, tooth hurt

- Follow-up treatment 
- Other

- Don't know

5. Check box if your child has or has ever had any of the following.

- Allergies

- Teething pain

- Other dental pain

- Heart disease/problems

- Cancer

- Hemophilia/bleeding problems

- A physically disabling condition

- A mentally disabling condition

6. Has anyone ever told you how to take care of your child's mouth?

- Yes

- No

- Don't know

If yes, whom?

- Dentist's office

- Physician's office

- Health Department

- Other

7. Which of the following best describes your child?

- White

- Black

- Asian or Pacific Islander

- American Indian or Alaskan

- Multiracial

- Hispanic/Latino

I give my consent for my child to participate in Smile KY! screening

- Yes

- No

After the questionnaires were collected each of the selected schools would be visited by several licensed dentists and hygienists who would conduct a dental screening on the children that the parents had given consent. The screening forms contained the following eleven questions:

1. Untreated cavities? Yes/No

2. Number of carious first permanent molars?

3. Number of quadrants needing caries treatment?

4. Existing restorations? Yes/No

5. Existing sealants on permanent molars? Yes/No

6. Number of missing permanent molars?

7. Oral injuries? Yes/No 
8. Malocclusion? Yes/No

9. Existing orthodontic appliances? Yes/No

10. Behavior?

- Could not follow directions

- Fearful, but cooperative

- No problem

11. Overall Oral Assessment

- Urgent care needed

- Fair, early stage dental disease

- Good, no obvious problem

\section{Data Manipulation}

Data from questionnaire and screening forms were added to the electronic database annually. Initially our sample size is of 38,740 children from 2002 to 2014 . After deleting missing values and duplicates observations $(2,580)$ the resulted patient count was 36,160 unique observations. In addition, data for the year 2012 was deleted. This year was an aberrant year because it snowed and all of our schools were not screened as planned, leaving 34,689 observations. Due to start-up difficulties in the year 2002 numbers were misleading and hence we eliminated data from 2002 for our analyses. This includes 1,257 observations comprising 3.62 percent of our original population $(\mathrm{N}=34,689)$. Our final sample comprised of data from 2003 to 2014 ( $\mathrm{N}=33,432$ observations) with 52 variables. We used SAS 9.3 statistical software for our data manipulation.

The variables include the child's demographic information such as age, gender, ethnicity and child's health history information including their insurance type and prior dental history. The variables from the screening form included presence of decay, carious first molars, restorations, oral hygiene status, anxiety level and overall Clinical Screening. In accordance with our research objective, we selected our variables of interest. Our primary risk factor was 'dental health instructions received' (yes/no) and outcome of 
interest was 'parental assessment' (good/fair/poor). All other variables were included in regression model. We used 'SAS 9.3' statistical software for data manipulation. Descriptive statistics, chi-square test of independence and multiple logistic regression modeling were performed on 'IBM SPSS Statistics 20' and 'R 3.1.1' statistical software at $p<0.05$.

\section{Research Design}

Our sample size was large with 33,432 children from 5 to 11 years of age. We designed a longitudinal cross-sectional study to conduct statistical analyses. We used SPSS statistical software to run descriptive statistics (table 1). Our outcome of interest was categorical with three levels and primary risk factor was a dichotomous variable. In order to identify the association between outcome and risk factor, both being categorical variables, we performed chi-square test of independence at a significance level of $\boldsymbol{p}<0.05$ and degree of freedom $=2[(3-1) *(2-1)]$. To perform a chi-square test, variables should be categorical with individual cell counts not less than five in a survey sample. We identified a significant relationship between parental assessment and oral health education received through healthcare providers.

To identify the healthcare provider with the most significant influence on parental assessment chi-square analysis was conducted between the four individual healthcare providers; dental office, physician office, healthcare department, and others; and the parental assessment (outcome of interest).

With an assumption that parents who received oral hygiene instructions from a healthcare provider would be better able to take care of their child's teeth, we expected a 
high percentage of 'good' Clinical Screening verified by a dentist. We performed chisquare tests between the Clinical screening and the type of healthcare provider.

Parental assessment of their child's oral health status was compared with the screening assessment based on having received oral health instructions from one of the four provider groups through individual chi-square analysis and plotted a bar graph for interpretation. We analyzed the total correct percentages and total wrong percentages, shown in results, and this answered our research objective. To increase validity and reliability of our results, we stratified our data year-wise from 2003 to 2014. Each year represented a unique dataset in our entire population. So for all the previously mentioned tests, we conducted trend analyses for parental assessment and clinical screening based on instructions received for 12 years $(2003,2004,2005,2006,2007,2008,2009,2010,2011$, 2013, and 2014, 2012 has 0 observations) and plotted error bars with standard errors identified, on the corresponding bars.

The Smile KY! screenings were conducted at different schools each year. Due to the large number of elementary schools in Jefferson County it took nine years to visit all of the elementary schools in the county. Bullitt County, being smaller, supported the Smile KY! effort and we were able to visit half of their schools every year. The other rural counties were visited every two years for screening and education. To identify the influence of repeated visits and unique visits, we performed chi-square analysis on Bullitt and Jefferson Counties separately. We stratified the entire dataset into two subsets based on school. Bullitt County included nine schools: Crossroads, freedom, Maryville, Nichols, Roby, Lebanon junction, Old mill, Pleasant grove and Mt.Washington. Jefferson County included the remaining schools. The total sample size was less for Bullitt County $(\mathrm{N}=4,598)$ 
compared with Jefferson County $(\mathrm{N}=28,834)$. A chi-square analysis with a year-by-year trend analysis was performed for both counties with standard errors plotted as error bars in corresponding graphs.

To identify the association between parental assessment and secondary risk factors, we intended to perform logistic regression modeling. Since parental assessment is a trinary variable and other predictors are categorical with yes or no options, we fitted multiple logistic regression model using SPSS statistical software. We performed "STEP" command on $\mathrm{R}$ statistical software to derive the best fitted model with the highest significance levels $(\mathrm{p}<0.000)$. Equation for multiple logistic regression model $^{42}$ was:

$$
\operatorname{logit}(p)=\log (p /(1-p))=\beta 0+\beta 1 * x 1+\beta 2 * x 2+\beta 3 * x 3+\beta 4 *_{x} 4+\ldots \ldots \ldots . . . k^{*} x k
$$

where $p$ determines the probability of outcome variable with failure/success with $0 / 1$ and $\mathrm{x} 1$, , $\mathrm{xk}$ represent individual predictor variables in the dataset. Then, $\beta 0, \beta 1, \ldots, \beta \mathrm{k}$ represent the maximum likelihood of outcome. Substituting x1, x2, ..xk with predictor variables form our study in the above equation, we get:

$\operatorname{logit}(p)=\log (p /(1-p))=\beta 0+\beta 1 *$ Instructions Dental Office $+\beta 2 *$ Instructions Health Department $+\beta 3 *$ Visit Reason $+\beta 4 *$ Insurance $+\ldots \ldots$

Initially, we included all study variables in the model. Based on the significance level $(\mathrm{p}<0.05)$, insignificant variables were eliminated step-wise. To determine accuracy of our regression model, we included classification table in our analysis. In addition to addressing our main research objective we, also, identified trends involved in caries percentage, inoffice dental visits and insurance over the past 12 years for the entire population along with Bullitt and Jefferson Counties. The results were interpreted in a yearly plot. 


\section{RESULTS AND DISCUSSION}

Our study population was large with 33,432 subjects. Table 1 contains descriptive statistics of our population. Male and females are distributed almost equally. The ethnic breakdown of the study population was: Caucasians (71.9\%), African Americans (15.4\%), Hispanics (4.4\%) and others including Asians, Pacific islanders, American Indian and Native Hawaiians $(8.3 \%)$. A large proportion of our study participants had a prior dental visit (93.1\%), the most common reason was for a regular check-up/exam (69.6\%).

A total of $27,543(82.4 \%)$ subjects received instructions from one of the listed healthcare providers, including dental office $(66.7 \%)$, physician office $(1.8 \%)$, other sources $(2.4 \%)$, health department $(1.1 \%)$ and a combination of two $(9.1 \%)$ or more providers $(1.3 \%)$. The remainder $(17.6 \%)$ of our participants had not received oral health education from any source. Interestingly, a corresponding proportion of our subjects had no insurance $(17.7 \%)$ coverage for dental services. Slightly less than fifty percent of our study participants had private insurance and the remainder had Medicaid or KCHIP. When

considering the health history questions, parents acknowledged the existence of teething pain $(13.8 \%)$, other dental pain $(7.5 \%)$, heart disease $(1.4 \%)$ and other physical condition including allergies (4.6\%). A nearly negligible percentage (1.3\%) of our study group had a mental condition such as autism, ADHD or other associated conditions.

The screening completed by a dentist revealed obvious caries in $30 \%$ of our study participants, with $5.2 \%$ exhibiting a large number of lesions and $4.5 \%$ demonstrated large 
sized carious lesions. Other indicators of caries such as carious first molars (14.4\%) and carious quadrants $(27.0 \%)$ reflect the caries incidence of this population. As a reflection of home care, our study population had poor oral hygiene (5.0\%), gingival inflammation (4.2\%), plaque (7.6\%) and calculus (1.2\%). Malocclusion was noted in $30.5 \%$ of the study population.

Medium and high anxiety levels were found in only $3 \%$ of the entire study population. Pertaining to dental treatments, our patient population had restorations (36.2\%), sealants $(25.1 \%)$ and orthodontic appliances (4.2\%) from previous dental intervention. Parental assessment felt $73.2 \%$ their child had good dental condition, while the remaining $23.7 \%$ rated their child at fair and $3.1 \%$ assessment was poor. Even though good and fair parental assessments are similar when compared to the dental clinician assessment, the poor $(6.1 \%)$ clinical screening is twice as many children as the poor parental assessment. 
Table 1: Descriptive characteristics of Study Population

\begin{tabular}{|c|c|c|c|c|}
\hline \multirow[t]{2}{*}{ Variable } & \multicolumn{3}{|c|}{ Parental Assessment } & \multirow[t]{2}{*}{ Total, N (\%) } \\
\hline & Good, N (\%) & Fair, N (\%) & Poor, N (\%) & \\
\hline $\begin{array}{l}\text { Gender } \\
\text { Male } \\
\text { Female }\end{array}$ & $\begin{array}{c}11,746(35.10) \\
12,711(38.0)\end{array}$ & $\begin{array}{c}4,089(12.2) \\
3,837(11.50)\end{array}$ & $\begin{array}{l}579(1.70) \\
470(1.40)\end{array}$ & $\begin{array}{l}16,414(49.1) \\
17,018(50.9)\end{array}$ \\
\hline $\begin{array}{l}\text { Ethnicity } \\
\text { White } \\
\text { Black } \\
\text { Hispanic } \\
\text { American Indian } \\
\text { Asian } \\
\text { Multiracial } \\
\text { Native Hawaiian } \\
\text { Other }\end{array}$ & $\begin{array}{c}18,306(54.80) \\
3,456(10.30) \\
806(2.4) \\
117(0.3) \\
366(1.1) \\
716(2.1) \\
27(0.1) \\
663(2.0)\end{array}$ & $\begin{array}{c}5,118(15.3) \\
1,503(4.5) \\
544(1.6) \\
47(0.1) \\
186(0.6) \\
319(1.0) \\
2(0.0) \\
207(0.6)\end{array}$ & $\begin{array}{c}614(1.8) \\
190(0.6) \\
129(0.4) \\
8(0.0) \\
33(0.1) \\
18(0.1) \\
2(0.0) \\
55(0.2)\end{array}$ & $\begin{array}{c}24,038(71.9) \\
5,149(15.4) \\
1,479(4.4) \\
172(0.5) \\
585(1.7) \\
1,053(3.1) \\
31(0.1) \\
925(2.8)\end{array}$ \\
\hline $\begin{array}{l}\text { Visited Dentist } \\
\text { No } \\
\text { Yes }\end{array}$ & $\begin{array}{c}1,398(4.2) \\
23,059(69.0)\end{array}$ & $\begin{array}{c}744(2.2) \\
7,182(21.5)\end{array}$ & $\begin{array}{l}174(0.5) \\
875(2.6)\end{array}$ & $\begin{array}{c}2,316(6.9) \\
31,116(93.1)\end{array}$ \\
\hline $\begin{array}{l}\text { Visit Reason } \\
\text { Check-up } \\
\text { Treatment } \\
\text { Something went wrong } \\
\text { Other } \\
\text { Don't Know }\end{array}$ & $\begin{array}{c}18,131(54.2) \\
1,229(3.7) \\
1,219(3.6) \\
471(1.4) \\
3,407(10.2)\end{array}$ & $\begin{array}{c}4,719(14.1) \\
606(1.8) \\
1,185(3.5) \\
296(0.9) \\
1,120(3.4)\end{array}$ & $\begin{array}{c}420(1.3) \\
90(0.3) \\
235(0.7) \\
50(0.1) \\
254(0.8)\end{array}$ & $\begin{array}{c}23,270(69.6) \\
1,925(5.8) \\
2,639(7.9) \\
817(2.4) \\
4,781(14.4)\end{array}$ \\
\hline $\begin{array}{l}\text { Dental Health Instruction } \\
\text { No } \\
\text { Yes }\end{array}$ & $\begin{array}{c}3,898(66.19) \\
20,559(74.64)\end{array}$ & $\begin{array}{l}1,683(28.58) \\
6,243(22.67)\end{array}$ & $\begin{array}{l}308(5.23) \\
741(2.69)\end{array}$ & $\begin{array}{c}5,889(17.61) \\
27,543(82.39)\end{array}$ \\
\hline $\begin{array}{l}\text { Insurance } \\
\text { Medicaid } \\
\text { KCHIP } \\
\text { Private } \\
\text { None } \\
\end{array}$ & $\begin{array}{c}5,644(16.9) \\
2,302(6.9) \\
12,661(37.9) \\
3,850(11.5)\end{array}$ & $\begin{array}{c}2,743(8.2) \\
868(2.6) \\
2,595(7.8) \\
1,720(5.1) \\
\end{array}$ & $\begin{array}{l}309(0.9) \\
117(0.3) \\
274(0.8) \\
349(1.0)\end{array}$ & $\begin{array}{c}8,696(26.0) \\
3,287(9.8) \\
15,530(46.5) \\
5,919(17.7) \\
\end{array}$ \\
\hline $\begin{array}{l}\text { Teething Pain } \\
\text { No } \\
\text { Yes }\end{array}$ & $\begin{array}{c}21,760(65.1) \\
2,697(8.1)\end{array}$ & $\begin{array}{c}6,327(18.9) \\
1,599(4.8)\end{array}$ & $\begin{array}{l}730(2.2) \\
319(1.0)\end{array}$ & $\begin{array}{c}28,817(86.2) \\
4,615(13.8)\end{array}$ \\
\hline $\begin{array}{l}\text { Other Dental Pain } \\
\text { No } \\
\text { Yes }\end{array}$ & $\begin{array}{c}23,103(69.1) \\
1,354(4.1)\end{array}$ & $\begin{array}{c}6,961(20.8) \\
965(2.9)\end{array}$ & $\begin{array}{l}848(2.5) \\
201(0.6)\end{array}$ & $\begin{array}{c}30,912(92.5) \\
2,520(7.5)\end{array}$ \\
\hline $\begin{array}{l}\text { Heart Disease } \\
\text { No } \\
\text { Yes }\end{array}$ & $\begin{array}{c}24,111(72.1) \\
346(1.0)\end{array}$ & $\begin{array}{c}7,815(23.4) \\
111(0.3)\end{array}$ & $\begin{array}{c}1,029(3.1) \\
20(0.1)\end{array}$ & $\begin{array}{c}32,955(98.6) \\
477(1.41)\end{array}$ \\
\hline $\begin{array}{l}\text { Physical Condition } \\
\text { No } \\
\text { Yes }\end{array}$ & $\begin{array}{c}23,439(70.1) \\
1,018(3.0)\end{array}$ & $\begin{array}{c}7,439(22.3) \\
487(1.5)\end{array}$ & $\begin{array}{c}1,005(3.0) \\
44(0.1)\end{array}$ & $\begin{array}{c}31,883(95.4) \\
1,549(4.6)\end{array}$ \\
\hline $\begin{array}{l}\text { Mental Condition } \\
\text { No } \\
\text { Yes }\end{array}$ & $\begin{array}{c}24,197(72.4) \\
260(0.8)\end{array}$ & $\begin{array}{c}7,794(23.3) \\
132(0.4)\end{array}$ & $\begin{array}{c}1,023(3.1) \\
26(0.1)\end{array}$ & $\begin{array}{c}32,014(98.7) \\
418(1.3)\end{array}$ \\
\hline $\begin{array}{l}\text { Cavities } \\
\text { No } \\
\text { Yes }\end{array}$ & $\begin{array}{c}18,442(78.8) \\
6,015(60.0)\end{array}$ & $\begin{array}{l}4,502(19.2) \\
3,424(34.1)\end{array}$ & $\begin{array}{l}461(2.0) \\
588(5.9)\end{array}$ & $\begin{array}{l}23,405(70.0) \\
10,027(30.0)\end{array}$ \\
\hline $\begin{array}{l}\text { Carious First Molars } \\
0 \\
1\end{array}$ & $\begin{array}{c}21,717(75.8) \\
1,331(62.9) \\
\end{array}$ & $\begin{array}{c}6,162(21.5) \\
686(32.4)\end{array}$ & $\begin{array}{c}754(2.6) \\
99(4.7) \\
\end{array}$ & $\begin{array}{c}28,633(85.6) \\
2,116(6.3) \\
\end{array}$ \\
\hline
\end{tabular}




\begin{tabular}{|c|c|c|c|c|}
\hline $\begin{array}{l}2 \\
3 \\
4\end{array}$ & $\begin{array}{l}772(55.7) \\
262(48.1) \\
375(49.9)\end{array}$ & $\begin{array}{l}525(37.9) \\
241(44.2) \\
312(41.5)\end{array}$ & $\begin{array}{l}89(6.4) \\
42(7.7) \\
65(8.6)\end{array}$ & $\begin{array}{l}1,386(4.1) \\
545(1.6) \\
752(2.2)\end{array}$ \\
\hline $\begin{array}{l}\text { Carious Quadrants } \\
0 \\
1 \\
2 \\
3 \\
4\end{array}$ & $\begin{array}{c}19,133(78.4) \\
2,518(66.9) \\
1,557(58.1) \\
610(51.7) \\
639(45.5)\end{array}$ & $\begin{array}{c}4,770(19.5) \\
1,115(29.6) \\
960(35.8) \\
485(41.1) \\
596(42.4)\end{array}$ & $\begin{array}{c}501(2.1) \\
130(3.5) \\
162(6.0) \\
86(7.3) \\
170(12.1)\end{array}$ & $\begin{array}{c}24,404(73.0) \\
3,763(11.3) \\
2,679(8.0) \\
1,181(3.5) \\
1,405(4.2)\end{array}$ \\
\hline $\begin{array}{l}\text { Restorations } \\
\text { No } \\
\text { Yes }\end{array}$ & $\begin{array}{c}16,311(76.5) \\
8,146(67.3)\end{array}$ & $\begin{array}{l}4,390(20.6) \\
3,536(29.2)\end{array}$ & $\begin{array}{l}621(2.9) \\
428(3.5)\end{array}$ & $\begin{array}{l}21,322(63.8) \\
12,110(36.2)\end{array}$ \\
\hline $\begin{array}{l}\text { Sealants } \\
\text { No } \\
\text { Yes } \\
\end{array}$ & $\begin{array}{c}17,804(71.1) \\
6,653(79.2) \\
\end{array}$ & $\begin{array}{l}6,352(25.4) \\
1,574(18.7) \\
\end{array}$ & $\begin{array}{l}875(3.5) \\
174(2.1) \\
\end{array}$ & $\begin{array}{c}25,031(74.9) \\
8,401(25.1) \\
\end{array}$ \\
\hline $\begin{array}{l}\text { Missing Molars } \\
0 \\
1 \\
2 \\
3 \\
4 \\
\end{array}$ & $\begin{array}{c}24,280(73.2) \\
64(65.3) \\
35(62.5) \\
04(57.1) \\
74(79.6) \\
\end{array}$ & $\begin{array}{l}7,869(23.7) \\
26(26.5) \\
14(25.0) \\
02(28.6) \\
15(16.1)\end{array}$ & $\begin{array}{c}1,029(3.1) \\
08(8.2) \\
07(12.5) \\
01(14.3) \\
04(4.3)\end{array}$ & $\begin{array}{c}33,178(99.2) \\
98(0.3) \\
56(0.2) \\
07(0.0) \\
93(0.3) \\
\end{array}$ \\
\hline $\begin{array}{l}\text { Ortho Appliance } \\
\text { No } \\
\text { Yes }\end{array}$ & $\begin{array}{c}23,437(73.1) \\
1,020(75.1)\end{array}$ & $\begin{array}{c}7,622(23.8) \\
304(22.4)\end{array}$ & $\begin{array}{c}1,014(3.2) \\
35(2.6)\end{array}$ & $\begin{array}{c}32,073(95.9) \\
1,359(4.1)\end{array}$ \\
\hline $\begin{array}{l}\text { Behavior } \\
\text { Low } \\
\text { Medium } \\
\text { High }\end{array}$ & $\begin{array}{c}23,788(73.4) \\
585(66.6) \\
84(66.7)\end{array}$ & $\begin{array}{l}7,642(23.6) \\
251(28.6) \\
33(26.2)\end{array}$ & $\begin{array}{l}997(3.1) \\
43(4.9) \\
09(7.1)\end{array}$ & $\begin{array}{c}32,427(97.0) \\
879(2.6) \\
126(0.4)\end{array}$ \\
\hline $\begin{array}{l}\text { Severity of Caries } \\
\text { No } \\
\text { Yes }\end{array}$ & $\begin{array}{c}23,524(73.7) \\
933(62.2)\end{array}$ & $\begin{array}{c}7,496(23.5) \\
430(28.7)\end{array}$ & $\begin{array}{l}912(2.9) \\
137(9.1)\end{array}$ & $\begin{array}{c}31,932(95.5) \\
1,500(4.5)\end{array}$ \\
\hline $\begin{array}{l}\text { Extent of Caries } \\
\text { No } \\
\text { Yes }\end{array}$ & $\begin{array}{c}23,357(73.7) \\
1,100(63.1)\end{array}$ & $\begin{array}{c}7,428(23.4) \\
498(28.6)\end{array}$ & $\begin{array}{l}905(2.9) \\
144(8.3)\end{array}$ & $\begin{array}{c}31,690(94.8) \\
1,742(5.2)\end{array}$ \\
\hline $\begin{array}{l}\text { Mouth Sores } \\
\text { No } \\
\text { Yes }\end{array}$ & $\begin{array}{c}24,377(73.2) \\
80(73.4)\end{array}$ & $\begin{array}{c}7,905(23.7) \\
21(19.3)\end{array}$ & $\begin{array}{c}1,041(3.1) \\
08(7.3)\end{array}$ & $\begin{array}{c}33,323(99.7) \\
109(0.3)\end{array}$ \\
\hline $\begin{array}{l}\text { Gingival Inflammation } \\
\text { No } \\
\text { Yes }\end{array}$ & $\begin{array}{c}23,417(73.1) \\
1,040(75.2) \\
\end{array}$ & $\begin{array}{c}7,650(23.9) \\
276(20.0) \\
\end{array}$ & $\begin{array}{c}982(3.1) \\
67(4.8) \\
\end{array}$ & $\begin{array}{c}32,049(95.9) \\
1,383(4.1) \\
\end{array}$ \\
\hline $\begin{array}{l}\text { Poor Oral Hygiene } \\
\text { No } \\
\text { Yes }\end{array}$ & $\begin{array}{c}23,293(73.3) \\
1,164(70.1)\end{array}$ & $\begin{array}{c}7,525(23.7) \\
401(24.1)\end{array}$ & $\begin{array}{c}953(3.0) \\
96(5.8)\end{array}$ & $\begin{array}{c}31,771(95.0) \\
1,661(5.0)\end{array}$ \\
\hline $\begin{array}{l}\text { Plaque } \\
\text { No } \\
\text { Yes }\end{array}$ & $\begin{array}{c}22,518(72.9) \\
1,939(76.0)\end{array}$ & $\begin{array}{c}7,416(24.0) \\
510(20.0)\end{array}$ & $\begin{array}{l}946(3.1) \\
103(4.0)\end{array}$ & $\begin{array}{c}30,880(92.4) \\
2,552(7.6)\end{array}$ \\
\hline $\begin{array}{l}\text { Calculus } \\
\text { No } \\
\text { Yes } \\
\end{array}$ & $\begin{array}{c}24,165(73.2) \\
292(70.9) \\
\end{array}$ & $\begin{array}{c}7,829(23.7) \\
97(23.5) \\
\end{array}$ & $\begin{array}{c}1,026(3.1) \\
23(5.6)\end{array}$ & $\begin{array}{c}33,020(98.8) \\
412(1.2)\end{array}$ \\
\hline $\begin{array}{l}\text { Malocclusion } \\
\text { No } \\
\text { Yes }\end{array}$ & $\begin{array}{c}17,617(75.0) \\
6,840(68.8)\end{array}$ & $\begin{array}{l}5,174(22.0) \\
2,752(27.7)\end{array}$ & $\begin{array}{l}692(2.9) \\
357(3.6)\end{array}$ & $\begin{array}{c}23,483(70.2) \\
9,949(29.8)\end{array}$ \\
\hline $\begin{array}{l}\text { Clinical Screening } \\
\text { Good } \\
\text { Fair }\end{array}$ & $\begin{array}{c}19,051(78.5) \\
4,571(64.1)\end{array}$ & $\begin{array}{l}4,740(19.5) \\
2,260(31.7)\end{array}$ & $\begin{array}{l}477(2.0) \\
302(4.2)\end{array}$ & $\begin{array}{c}24,268(72.6) \\
7,133(21.3)\end{array}$ \\
\hline
\end{tabular}




\begin{tabular}{|l|c|c|c|c|}
\hline Poor & $835(41.1)$ & $926(45.6)$ & $270(13.3)$ & $2,031(6.1)$ \\
\hline Total & $\mathbf{2 4 , 4 5 7 ( 7 3 . 1 5 )}$ & $\mathbf{7 , 9 2 6}(\mathbf{2 3 . 7 1})$ & $\mathbf{1 , 0 4 9}(\mathbf{3 . 1 4})$ & $\mathbf{3 3 , 4 3 2 ( 1 0 0 . 0 0 )}$ \\
\hline
\end{tabular}

Figure 1: Frequency of Parental Assessment

\section{Parental Assessment}

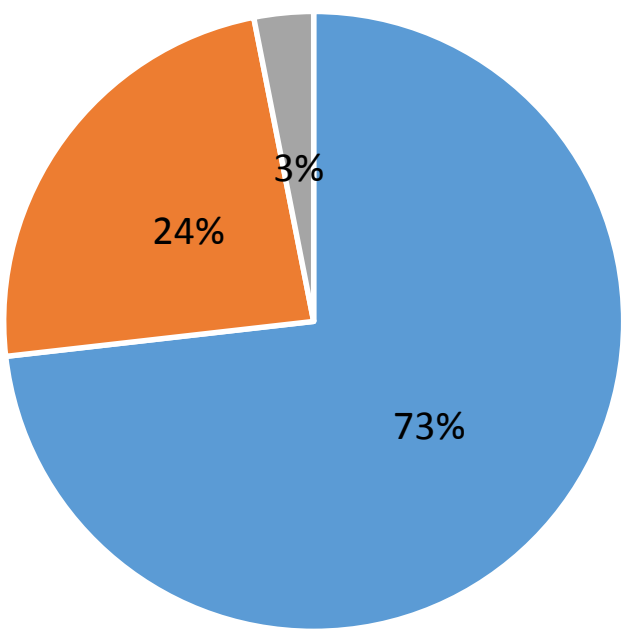

- Good $\square$ Fair $\square$ Poor

Table 2 and figure 2 demonstrates the frequencies of different providers involved in parental oral health education compared to the child's dental status. The healthcare providers include dental office $(66.7 \%)$, physician office $(1.8 \%)$, health department $(1.1 \%)$ and other provider sources $(2.4 \%)$ which would include nurse practitioners, school nurses, other school personnel, friends/neighbors, and information received from TV and print media. As seen in Table 2 parents received oral health instructions from a combination of two $(8.3 \%)$, three $(0.6 \%)$ and four $(0.3 \%)$ healthcare providers. A total of $5,889(17.6 \%)$ of our study population answered 'no' for dental health instructions received, which is our primary risk factor. 
Table 2: Frequency of Provider Source

\begin{tabular}{|l|l|c|c|}
\hline $\begin{array}{c}\text { Table } \\
\text { Graph }\end{array}$ & \multicolumn{1}{|c|}{ Source } & Count, N & $\begin{array}{c}\text { Percentage, } \\
(\boldsymbol{\%})\end{array}$ \\
\hline 1. & Dental Office Only & 22,285 & 66.66 \\
\hline 2. & Physician Office Only & 584 & 1.75 \\
\hline 3. & Health Department Only & 355 & 1.06 \\
\hline 4. & Other Only & 815 & 2.44 \\
\hline 5. & Dental Office \& Health Department & 246 & 0.74 \\
\hline 6. & Dental Office \& Other & 714 & 2.14 \\
\hline 7. & Dental Office \& Physician Office & 1981 & 5.93 \\
\hline 8. & Health Department \& Other & 25 & 0.07 \\
\hline 9. & Health Department \& Physician Office & 27 & 0.08 \\
\hline 10. & Physician Office \& Other & 33 & 0.10 \\
\hline 11. & Dental Office, Health Department \& Other & 29 & 0.09 \\
\hline 12. & $\begin{array}{l}\text { Health Department, Other \& Physician } \\
\text { Office }\end{array}$ & 4 & 0.01 \\
\hline 13. & Dental Office, Other \& Physician Office & 145 & 0.43 \\
\hline 14. & $\begin{array}{l}\text { Dental Office, Health Department \& } \\
\text { Physician Office }\end{array}$ & 29 & 0.09 \\
\hline 15. & All & 84 & 0.25 \\
\hline 16. & None & 5889 & 17.61 \\
\hline
\end{tabular}


Figure 2: Provider Source Distribution

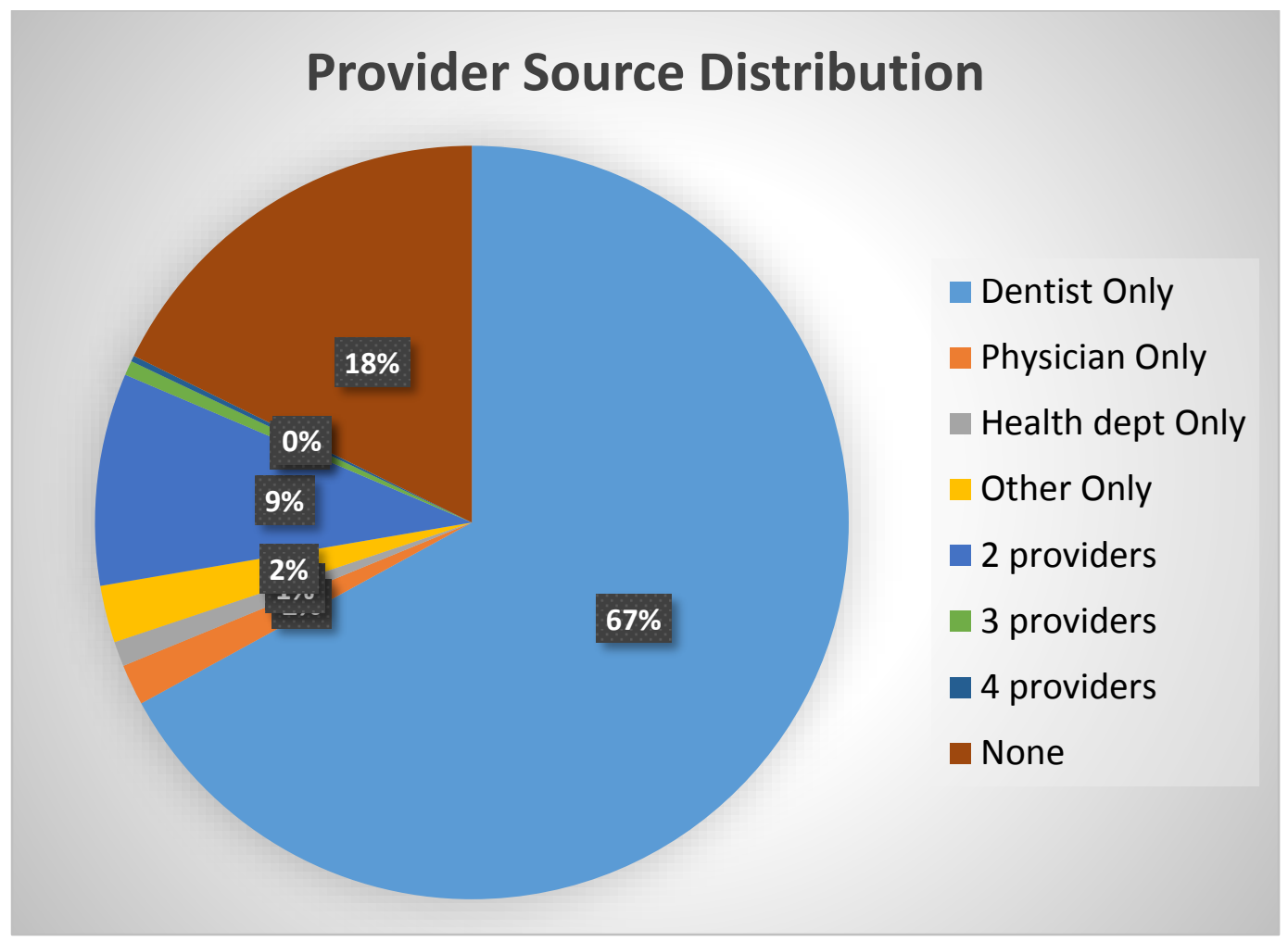


As evidenced in Table 3, a significant p-value of $<0.0001$ indicates that parental assessment was significantly associated with oral health education received from a healthcare professionally. The probability of $<1$ in chi-square test accepts our hypothesis; concluding that parental assessment is dependent on oral health training.

We calculated standard errors for all the years data was collected together to include error points in the bar graph. From which, we are $95 \%$ confident that our observed values are in close proximity to the actual values

Table 3: Chi-square Test of Independence Between Dental Health Education and Parental Assessment

\begin{tabular}{|c|c|c|c|c|c|}
\hline \multirow{2}{*}{$\begin{array}{c}\text { Dental } \\
\text { Health } \\
\text { Instruction }\end{array}$} & \multicolumn{3}{|c|}{ Parental Assessment } & Total, N (\%) & \multirow{2}{*}{$\begin{array}{c}\text { P- } \\
\text { Value }\end{array}$} \\
\cline { 2 - 4 } & Good, N (\%) & Fair, N (\%) & $\begin{array}{c}\text { Poor, N } \\
(\%)\end{array}$ & \\
\hline No & $3,898(66.19)$ & 1,683 & $308(5.23)$ & $5,889(17.61)$ & $<0.0001$ \\
& 20,559 & 6,243 & $741(2.69)$ & 27,543 & \\
Yes & $(74.64)$ & $(22.67)$ & & $(82.39)$ & \\
& 24,457 & 7,926 & 1,049 & 33,432 & \\
\hline Total & $(73.15)$ & $(23.71)$ & $(3.14)$ & $(100.0)$ & \\
& & & &
\end{tabular}

Figure 3: Relation Between Parental Assessment and Oral Health Education

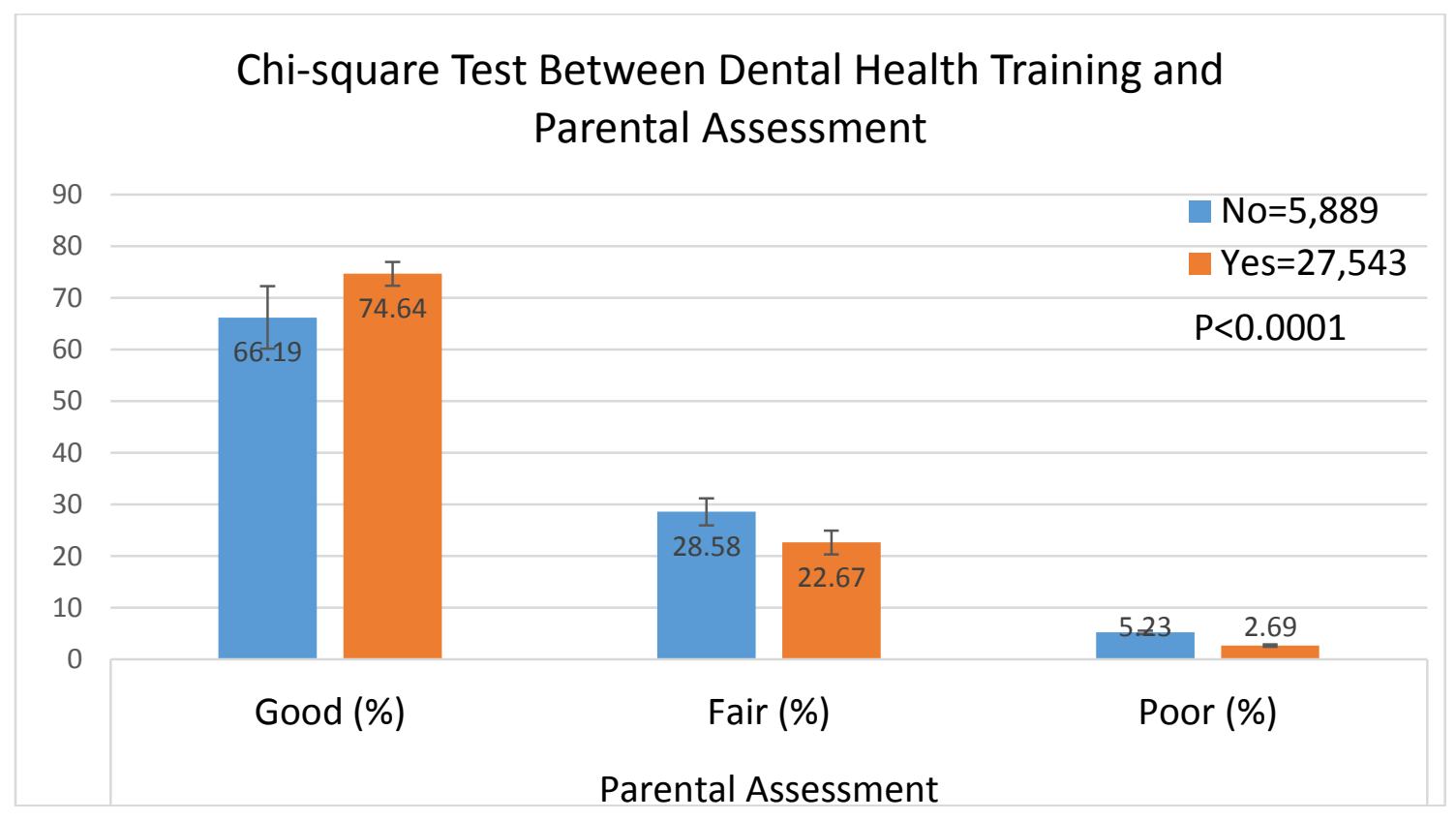


From Table 4, $\mathrm{P}=0.0008$ is less than the significance level of 0.05 . This shows that our outcome and risk factor are significantly associated even with a subset of population that represents repeated dental visits. In addition, small numbers of standard error bars increase our research reliability and validity. Compared to good and fair parental assessments, standard error for poor parental assessment is most significant. This further reflects the influence of oral health education on parental ratings.

Table 4: Chi-square Test Between Dental Health Training and Parental Assessment - Bullitt County

\begin{tabular}{|c|c|c|c|c|c|}
\hline \multirow{2}{*}{$\begin{array}{c}\text { Dental } \\
\text { Health } \\
\text { Instruction }\end{array}$} & \multicolumn{3}{|c|}{ Parental Assessment } & Total, N (\%) & \multirow{2}{*}{$\begin{array}{c}\text { P- } \\
\text { Value }\end{array}$} \\
\cline { 2 - 5 } & Good, N (\%) & Fair, N (\%) & Poor, N (\%) & & \\
\hline No & $519(73.72)$ & $160(22.73)$ & $25(3.55)$ & $704(15.31)$ & 0.0008 \\
\hline Yes & $3,113(79.94)$ & $687(17.64)$ & $94(2.41)$ & $3,894(84.69)$ & \\
\hline Total & $3,632(78.99)$ & $847(18.42)$ & $119(2.59)$ & $4,598(100.00)$ & \\
\hline
\end{tabular}

Figure 4: Relation Between Oral Health Education and Parental Assessments in Bullitt County (subset with multiple dental visits)

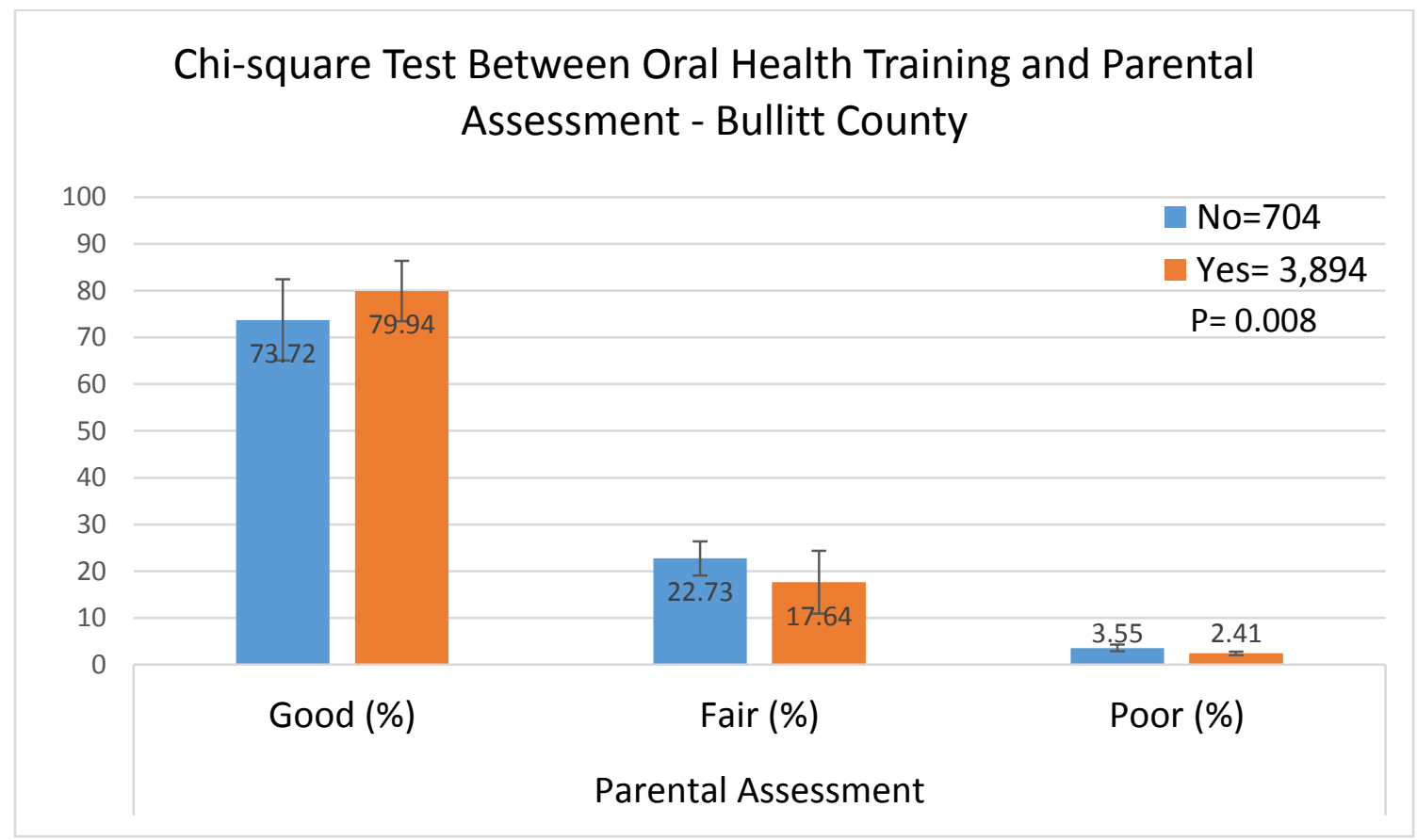


From Table 5, $\mathrm{P}<0.0001$ is far less than the significance level of 0.05 . This shows that our outcome and risk factor are significantly associated even with a subset of population that represents children with unique dental visits through Smile KY! screenings. Additionally, small numbers of standard error bars increase our research reliability and validity. Compared to good and fair parental assessments, standard error for poor parental assessment is most significant. This further reflects the influence of oral health education on parental ratings.

Table 5: Chi-square Test Between Dental Health Training and Parental Assessment - Jefferson County

\begin{tabular}{|c|c|c|c|c|c|}
\hline \multirow{2}{*}{$\begin{array}{c}\text { Dental } \\
\text { Health } \\
\text { Instruct }\end{array}$} & \multicolumn{3}{|c|}{ Parental Assessment } & Total, N (\%) & $\begin{array}{c}\text { P- } \\
\text { Value }\end{array}$ \\
\cline { 2 - 4 } & Good, N (\%) & Fair, N (\%) & Poor, N (\%) & \\
\hline No & $3,379(65.17)$ & $1,523(29.37)$ & $283(5.46)$ & $5,185(17.98)$ & $<0.000$ \\
\hline Yes & $17,446(73.77)$ & $5,556(23.49)$ & $647(2.74)$ & $23,649(82.02)$ & 1 \\
\hline Total & $20,825(72.22)$ & $7,079(24.55)$ & $930(3.23)$ & $28,834(100.00)$ & \\
\hline
\end{tabular}

Figure 5: Relation Between Oral Health Education and Parental Assessments in Jefferson County (subset with unique dental visits)

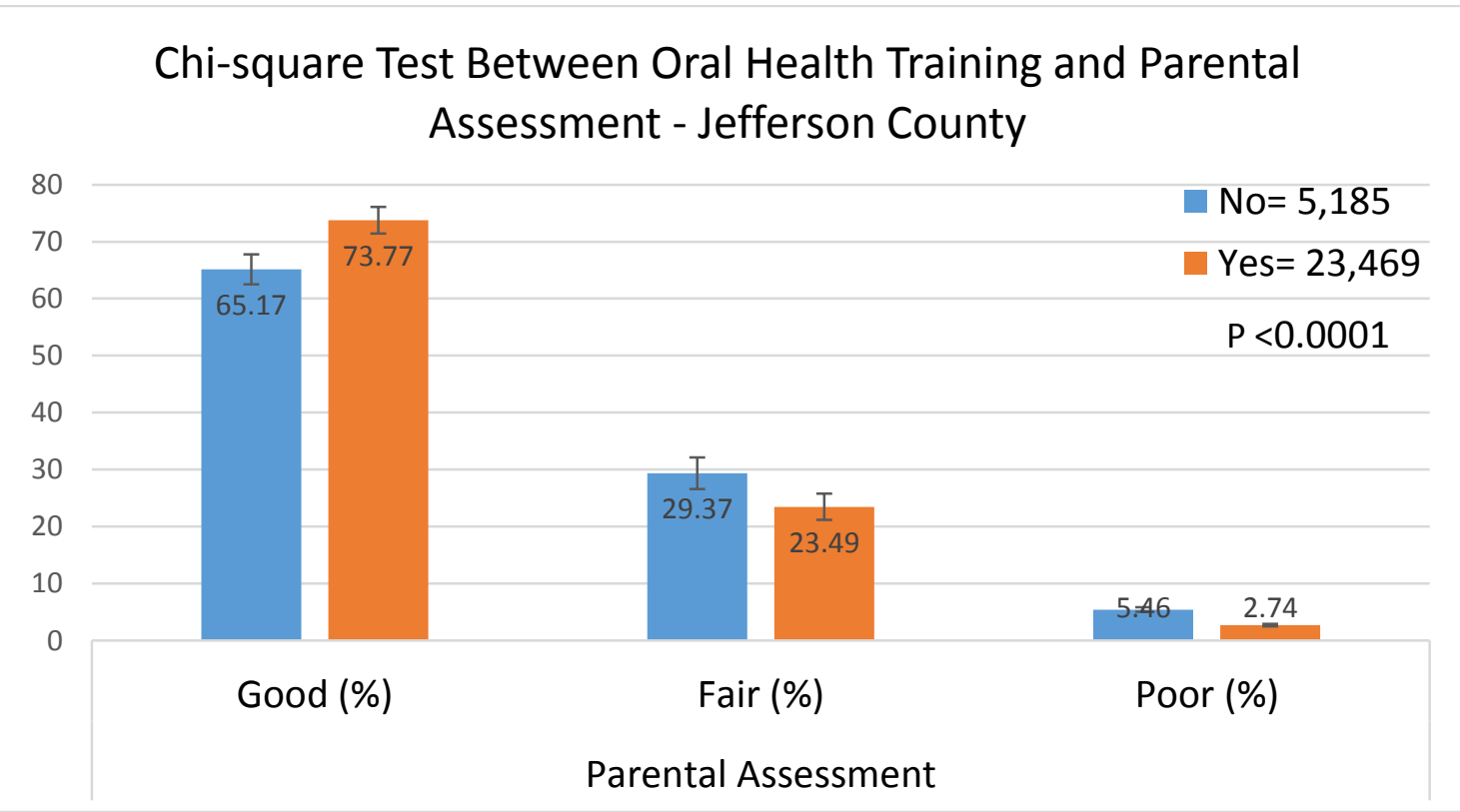


From Table 6, $\mathrm{P}<0.0001$ is far below the significance level of 0.05 . This shows that instructions received in a dental office are significantly associated with parental assessments. As shown on Figure 6, good parental assessment is more than fair and poor. Standard errors calculated at 95\% Confidence Interval show that the calculated mean for poor parental assessment is less than two percent in deviation with the mean of overall population.

Table 6: Chi-square Test Between Dental Office Instructions and Parental Assessment

\begin{tabular}{|c|c|c|c|c|c|}
\hline \multirow{2}{*}{$\begin{array}{c}\text { Dental } \\
\text { Health } \\
\text { Instruction }\end{array}$} & \multicolumn{3}{|c|}{ Parental Assessment } & \multirow{2}{*}{ Total (\%) } & $\begin{array}{c}\text { P- } \\
\text { Value }\end{array}$ \\
\cline { 2 - 5 } & Good (\%) & Fair (\%) & Poor (\%) & & \\
\hline No & $3,898(66.19)$ & $1,683(28.58)$ & $308(5.23)$ & $5,889(20.90)$ & $<0.0001$ \\
\hline Yes & $16,811(75.44)$ & $4,961(22.26)$ & $513(2.30)$ & $22,285(79.10)$ & \\
\hline Total & $20,709(73.5)$ & $6,644(23.58)$ & $821(2.91)$ & 28,174 & \\
& & & & $(100.00)$ & \\
\hline
\end{tabular}

Figure 6: Relation Between Parental Assessment and Oral Health Education from Dental Office

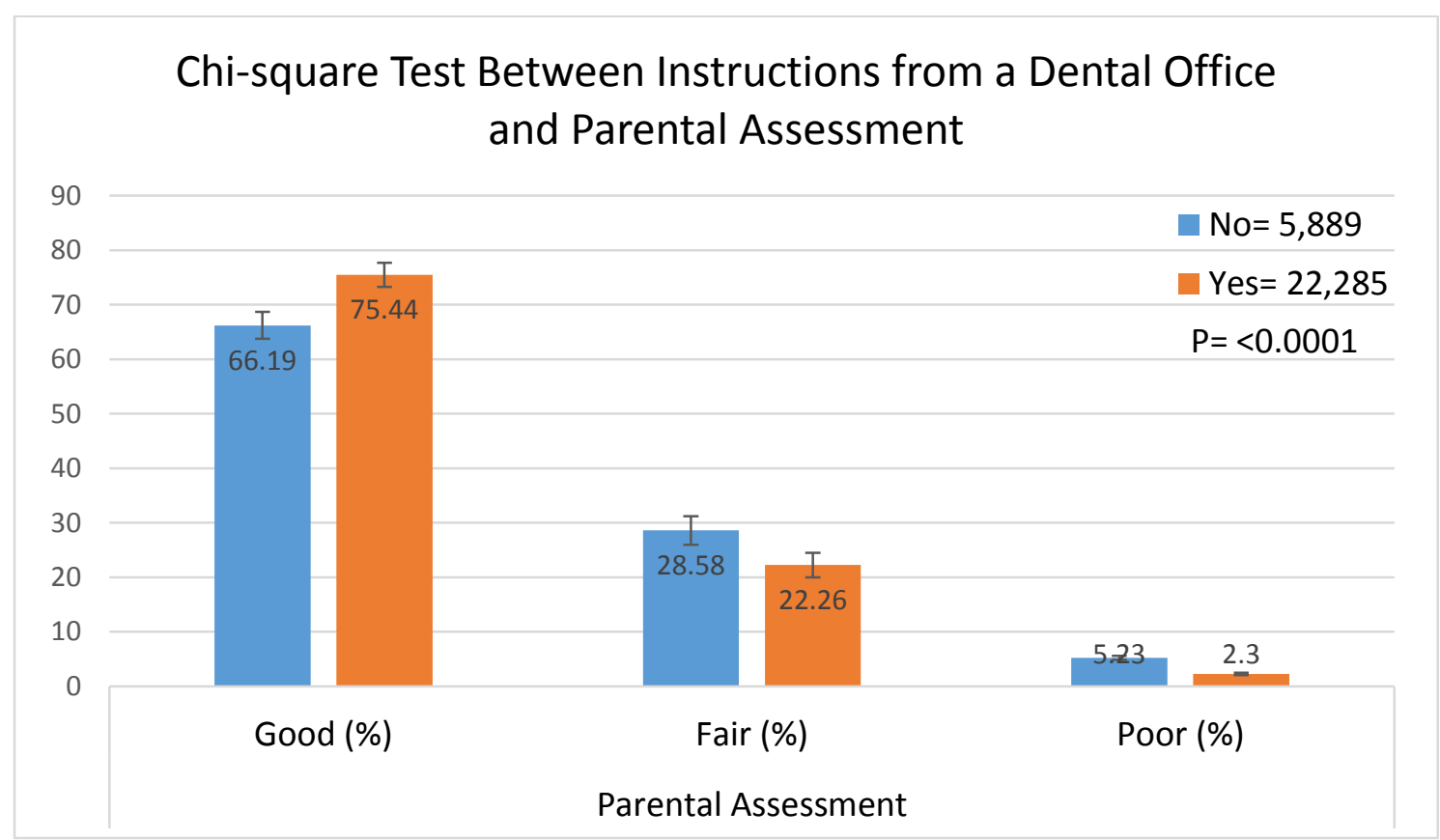


From Table $7, \mathrm{P}=0.59$ is above the significance level of 0.05 . This shows that instructions received from a physician office are in weak association with parental assessments. Chi-square p-value of $<1$ indicates the dependence of parental assessment on instructions from physician office. As demonstrated on Figure 7, when compared with no dental health education, little change can be seen for good parental assessment with prior instructions. Standard errors calculated at $95 \%$ Confidence Interval, shows that the mean for poor parental assessment is close to the mean of overall population.

Table 7: Chi-square Test Between Physician Office Instructions and Parental Assessment

\begin{tabular}{|c|c|c|c|c|c|}
\hline \multirow{2}{*}{$\begin{array}{c}\text { Physician } \\
\text { Office } \\
\text { Instruction }\end{array}$} & \multicolumn{3}{|c|}{ Parental Assessment } & Total, N (\%) & P- \\
\cline { 2 - 4 } & Good, N (\%) & Fair, N (\%) & Poor, N (\%) & & Value \\
\hline No & $3,898(66.19)$ & $1,683(28.58)$ & $308(5.23)$ & $5,889(90.98)$ & 0.5984 \\
\hline Yes & $388(66.44)$ & $171(29.28)$ & $25(4.28)$ & $584(9.02)$ & \\
\hline Total & $4,286(66.21)$ & $1,854(28.64)$ & $333(5.14)$ & $6,473(100.00)$ & \\
\hline
\end{tabular}

Figure 7: Relation Between Parental Assessment and Oral Health Education from Physician Office

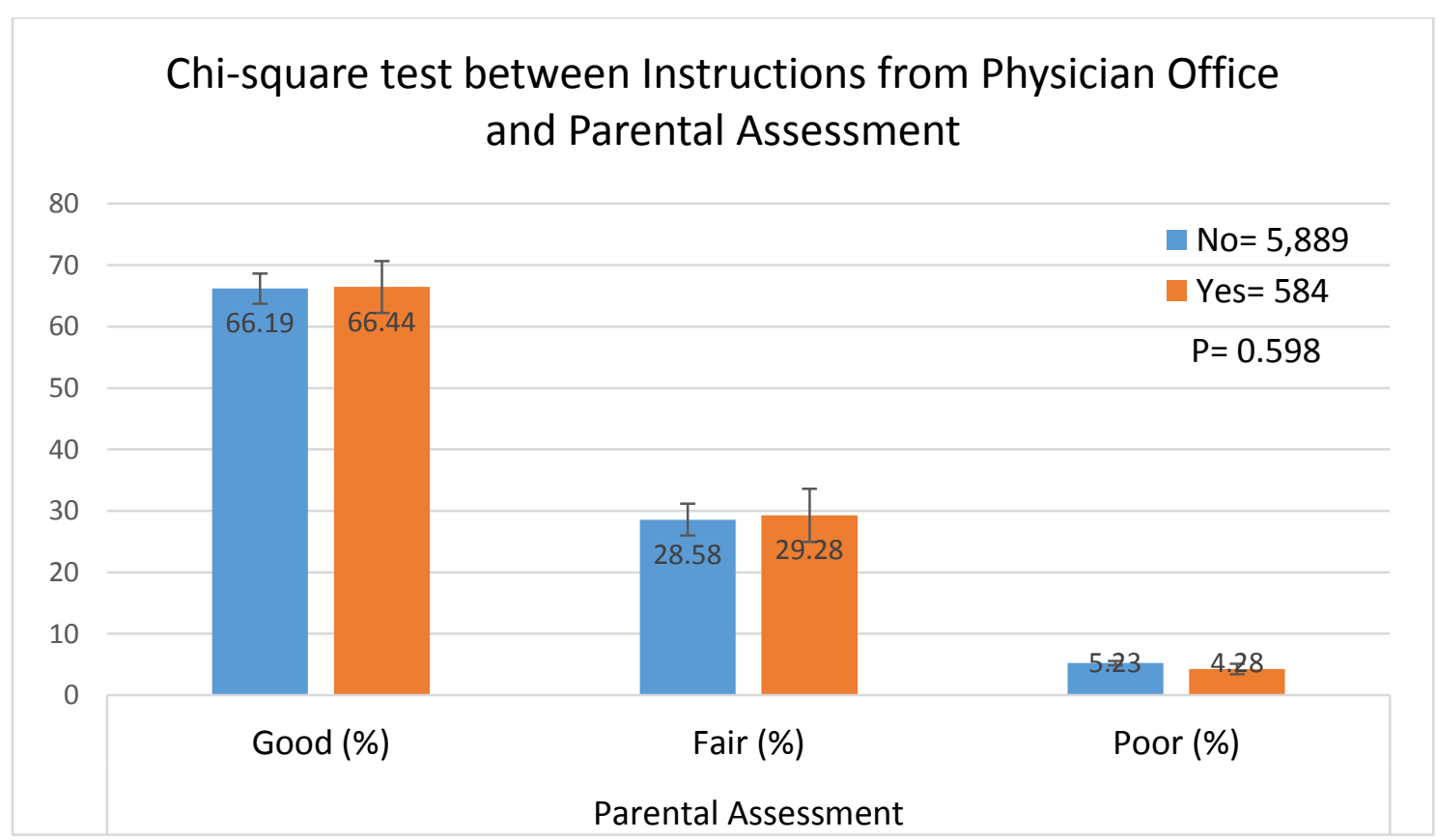


From Table $8, \mathrm{P}=0.0003$ is far below the significance level of 0.05 . This shows that oral health instructions from a health department are strongly associated with parental assessments. As evidenced from Figure 8, approximately an 8\% discrepancy can be identified for 'good' assessments with instructions from health department. Further, twofifths of population with oral health education from health department rated their child's dentition as fair and poor parental ratings.

Table 8: Chi-square Test Between Health Department and Parental Assessment

\begin{tabular}{|c|c|c|c|c|c|}
\hline \multirow{2}{*}{$\begin{array}{c}\text { Health } \\
\text { Department } \\
\text { Instruction }\end{array}$} & \multicolumn{3}{|c|}{ Parental Assessment } & Total, N (\%) & P- \\
\cline { 2 - 5 } & Good, N (\%) & Fair, N (\%) & Poor, N (\%) & & \\
\hline No & $3,898(66.19)$ & $1,683(28.58)$ & $308(5.23)$ & $5,889(94.31)$ & 0.0003 \\
\hline Yes & $205(57.75)$ & $117(32.96)$ & $33(9.30)$ & $355(5.69)$ & \\
\cline { 1 - 4 } Total & $4,103(65.71)$ & $1,800(28.83)$ & $341(5.46)$ & 6,244 & \\
& & & & $(100.00)$ & \\
\hline
\end{tabular}

Figure 8: Relation Between Parental Assessment and Oral Health Education from Health Department

\section{Chi-square Test Between Instructions from Health Department and Parental Assessment}

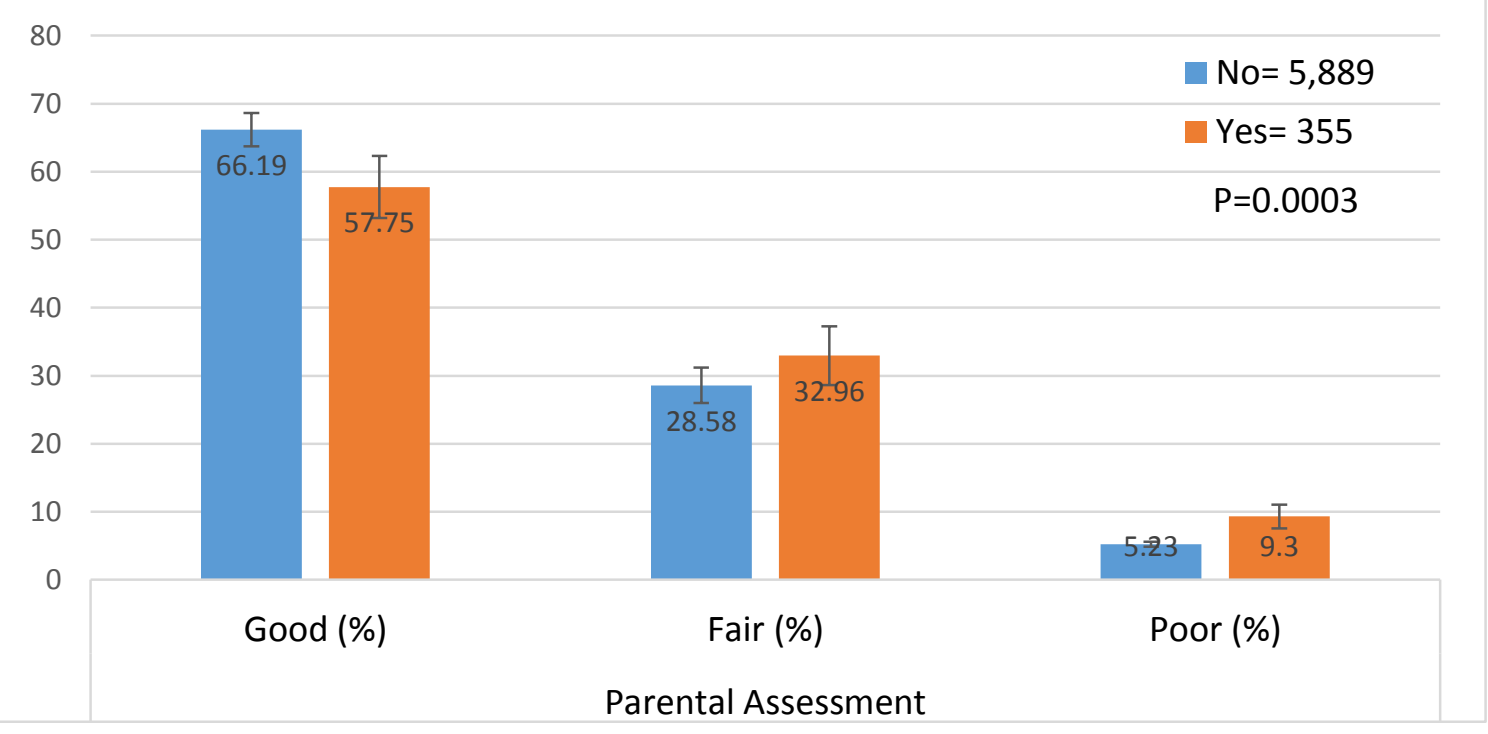


Outcomes from Table 9, $\mathrm{P}=0.0012$ are far below the significance level of 0.05 . This shows that instructions received from 'other' providers are significantly associated with parental assessments. The influence is similar to that of oral health education from a health department. As evident from Figure 9 shows that approximately $40 \%$ of the parents who received dental education from 'other' providers rated their child's dental condition as 'fair' and 'poor'.

Table 9: Chi-square Test Between 'Other' Provider Sources and Parental Assessment

\begin{tabular}{|c|c|c|c|c|c|}
\hline \multirow{2}{*}{$\begin{array}{c}\text { Other } \\
\text { Instruction }\end{array}$} & \multicolumn{3}{|c|}{ Parental Assessment } & \multirow[t]{2}{*}{ Total $(\%)$} & \multirow{2}{*}{$\begin{array}{c}\text { P- } \\
\text { Value }\end{array}$} \\
\hline & Good, N (\%) & Fair, N (\%) & $\begin{array}{c}\text { Poor, } \mathrm{N} \\
(\%)\end{array}$ & & \\
\hline No & $3898(66.19)$ & $1683(28.58)$ & $308(5.23)$ & $5889(87.84)$ & \multirow[t]{3}{*}{0.0012} \\
\hline Yes & $491(60.25)$ & $264(32.39)$ & $60(7.36)$ & $815(12.16)$ & \\
\hline Total & $4389(65.47)$ & $1947(29.04)$ & $368(5.49)$ & $6704(100.00)$ & \\
\hline
\end{tabular}

Figure 9: Relation Between Parental Assessment and Oral Health Education from 'Other' Provider Sources

\section{Chi-square Test Between Instructions from Other \\ Providers and Parental Assessment}

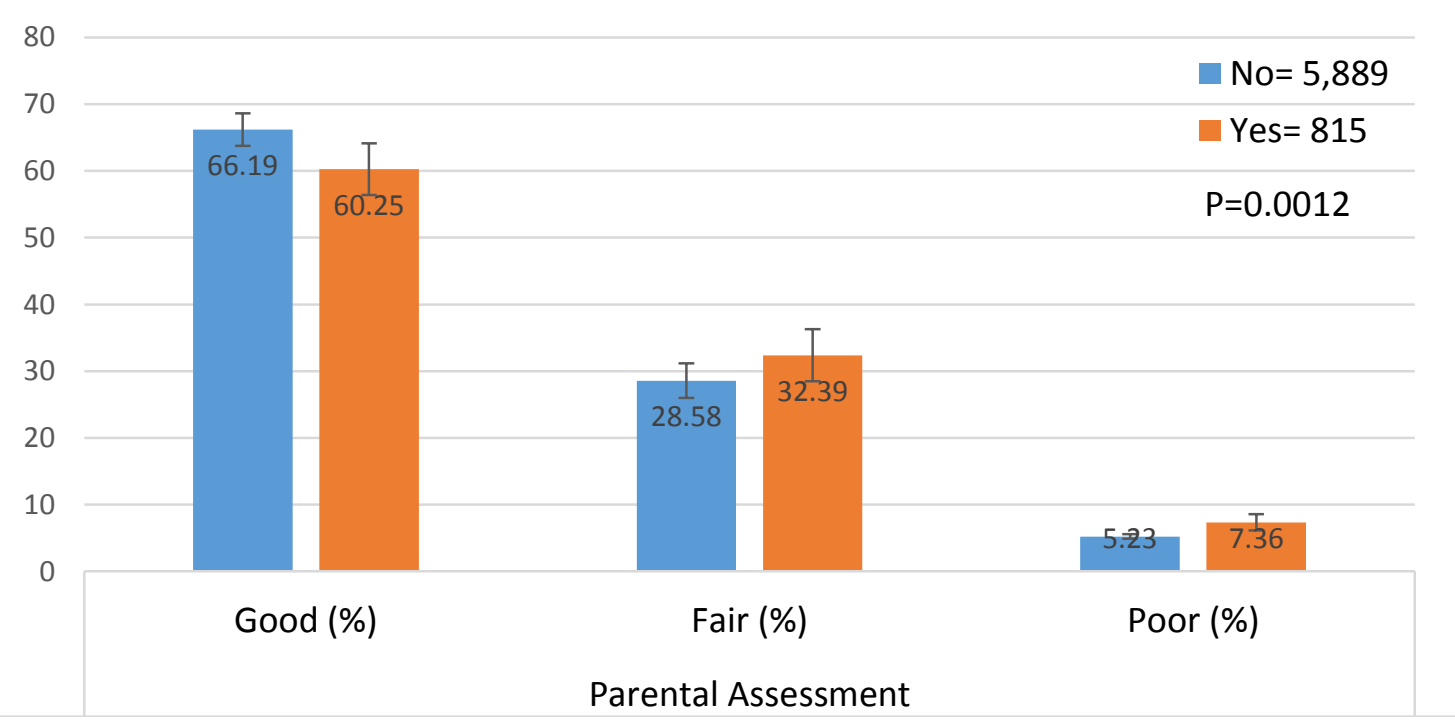


Table 10, $\mathrm{P}<0.0001$ outcomes are far below the significance level of 0.05 . This shows that dental health training is significantly associated with clinical screening done by a licensed dental practitioner. With the assumption that parents who received oral hygiene instructions from multiple providers are more knowledgeable and better able to take care of their child's teeth, we expected a high percentage of good clinical screenings. Table 10 and Figure 10 show that $73.9 \%$ of the parents with oral-health training stated their children had good dental health. This further substantiates our assumption with strong statistical support. We are $95 \%$ confident that there exists a strong association between clinical screening and oral health education received.

Table 10: Chi-square Test Between Dental Health Training and Clinical Screening

\begin{tabular}{|c|c|c|c|c|c|}
\hline \multirow{2}{*}{$\begin{array}{c}\text { Dental } \\
\text { Health } \\
\text { Instruct }\end{array}$} & \multicolumn{3}{|c|}{ Clinical Screening } & Total, N (\%) & $\begin{array}{c}\text { P- } \\
\text { Valu } \\
\text { e }\end{array}$ \\
\hline No & $3,922(66.60)$ & $1,481(25.15)$ & $486(8.25)$ & $5,889(17.61)$ & $<0.00$ \\
\hline Yes & $20,346(73.87)$ & $5,652(20.52)$ & $1,545(5.61)$ & $27,543(82.39)$ & 01 \\
\hline Total & $24,268(72.59)$ & $7,133(21.34)$ & $2,031(6.08)$ & $33,432(100.00)$ & \\
\hline
\end{tabular}

Figure 10: Relation Between Clinical Screening and Oral Health Education

\section{Chi-square Test Between Dental Health Training and Clinical Screening}

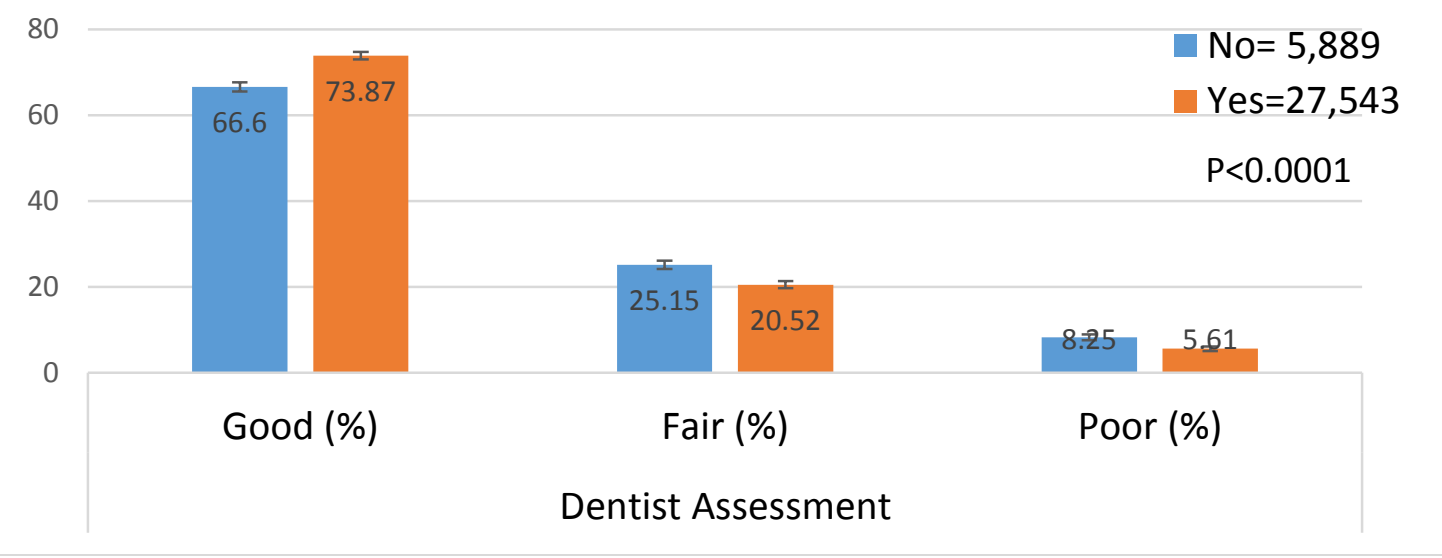


Findings from Table 11 support the results form Table 10. Similar chi-square analysis was done on the population with multiple dental visits. As seen, $\mathrm{P}<0.0001$ shows that parenteral assessments done after dental health training are significantly associated with the Clinical Screening done by a licensed dental practitioner. As expected, only onefourth of parents with oral health education rated their child's oral health as 'fair' or 'poor'. This further reflects the influence of oral health education on parental knowledge of oral health and their ability to identify dental problems in their child's mouth.

Table 11: Chi-square Test Between Dental Health Training and Clinical Screening Bullitt County

\begin{tabular}{|c|c|c|c|c|c|}
\hline \multirow{2}{*}{$\begin{array}{c}\text { Dental } \\
\text { Health } \\
\text { Instruction }\end{array}$} & \multicolumn{3}{|c|}{ Clinical Screening } & \multirow[t]{2}{*}{ Total, N (\%) } & \multirow{2}{*}{$\begin{array}{c}\text { P- } \\
\text { Value }\end{array}$} \\
\hline & Good, N (\%) & Fair, N (\%) & Poor, N (\%) & & \\
\hline No & $460(65.34)$ & $178(25.28)$ & $66(9.38)$ & 704 (15.31) & \multirow[t]{3}{*}{$<0.0001$} \\
\hline Yes & $2,905(74.60)$ & 741 (19.03) & $248(6.37)$ & $3,894(84.69)$ & \\
\hline Total & $3,365(73.18)$ & 919 (19.99) & $314(6.83)$ & $4,598(100.00)$ & \\
\hline
\end{tabular}

Figure 11: Relation Between Clinical Screening and Oral Health Education from Dental Office for Bullitt County (Subset with repeated dental visits)

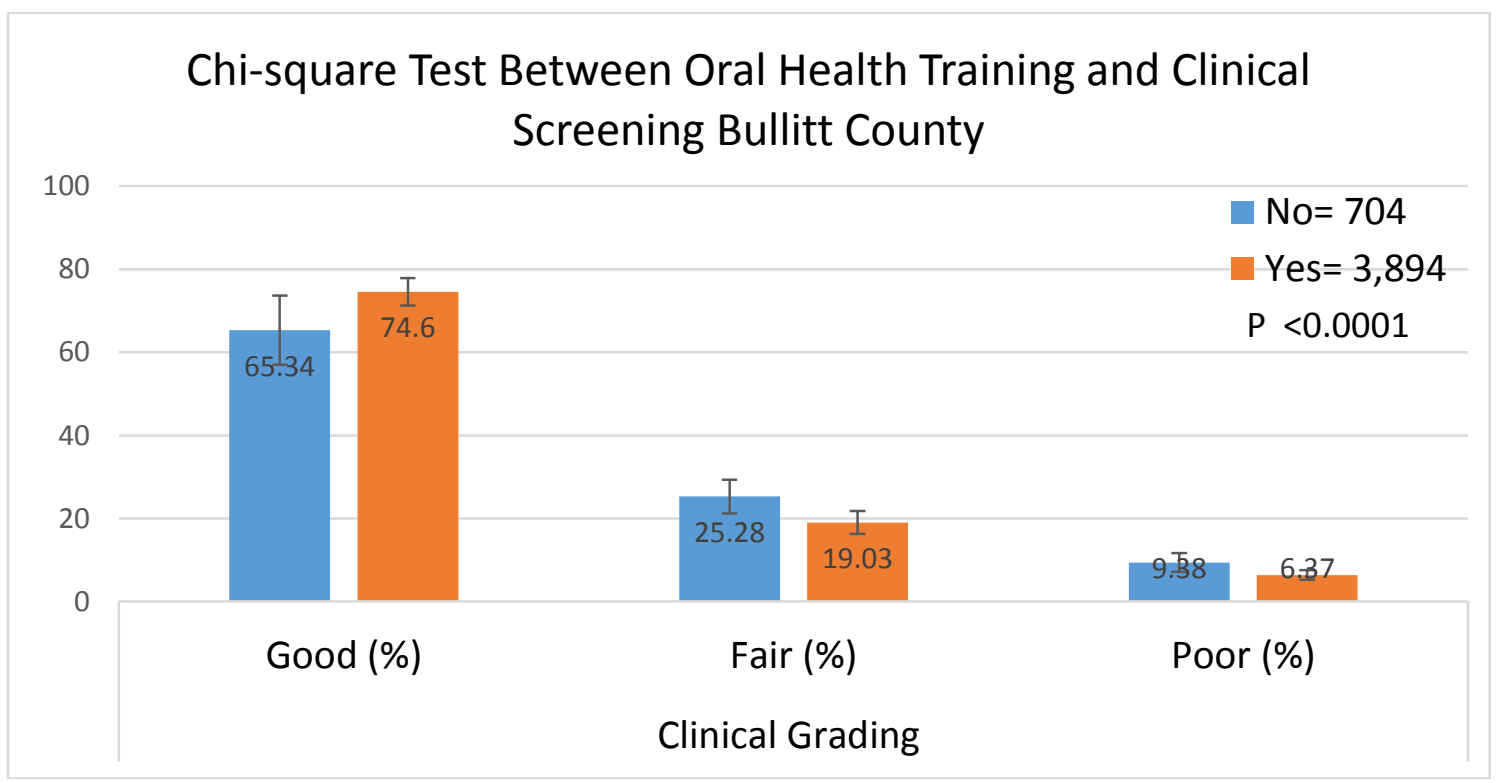


Data from Table 12, $\mathrm{P}<0.0001$ is far below the significance level of 0.05 . This shows that dental health training is significantly associated with the Clinical Screening done by a licensed dental practitioner. Jefferson County with primarily unique patient visits had similar findings to Bullitt County with repeated visits. When comparing parent assessments with prior dental health education to parent assessments without dental education, fair and poor Clinical Screenings were 7\% more among children of parents with no dental education. Table 12 and Figure 12 substantiates our assumption that educated parents make better assessments with a good evidence. In addition to the p-value, standard errors calculated from 12 years increases the reliability of our findings.

Table 12: Chi-square Test Between Dental Health Training and Clinical Screening Jefferson County

\begin{tabular}{|c|c|c|c|c|c|}
\hline \multirow{2}{*}{$\begin{array}{c}\text { Dental } \\
\text { Health } \\
\text { Instruct }\end{array}$} & \multicolumn{3}{|c|}{ Clinical Screening } & Total, N (\%) & $\begin{array}{c}\text { P- } \\
\text { Valu } \\
\text { e }\end{array}$ \\
\cline { 2 - 5 } & Good, N (\%) & Fair, N (\%) & Poor, N (\%) & & \\
\hline No & $3,462(66.77)$ & $1,303(25.13)$ & $420(8.10)$ & $5,185(17.98)$ & $<0.00$ \\
\hline Yes & $17,441(73.75)$ & $4,911(20.77)$ & $1,297(5.48)$ & $23,649(82.02)$ & 01 \\
\hline Total & $20,903(72.49)$ & $6,214(21.55)$ & $1,717(5.95)$ & $28,834(100.00)$ & \\
\hline
\end{tabular}

Figure 12: Relation Between Clinical Screening and Oral Health Education for Jefferson County (Subset with unique dental visits)

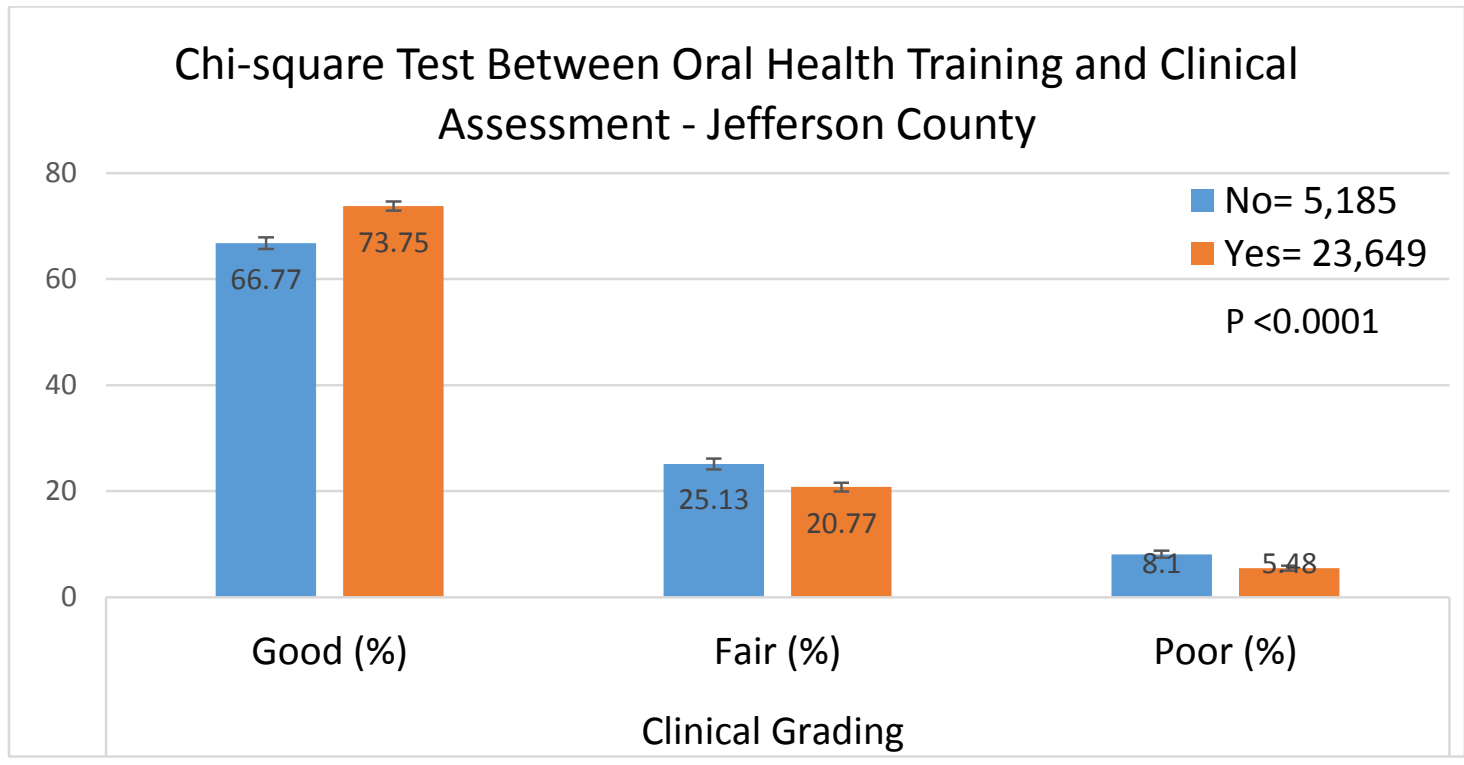


From Table 13, the significant p-value reflects a strong association between dental health training and a Clinical Screening done by a licensed dental practitioner. For parent assessments done by parents with dental education, 'good' parental assessment were approximately $8 \%$ more than those without training from a dental office. Figure 13 shows a corresponding reduction of $8 \%$ in 'fair' and 'poor' parental assessments from parents with oral health training from a dental office. Standard errors calculated at $95 \%$ Confidence Interval and show that the findings are reliable with less than two percent error.

Table 13: Chi-square Test Between Dental Office Instructions and Clinical Screening

\begin{tabular}{|c|c|c|c|c|c|}
\hline \multirow{2}{*}{$\begin{array}{c}\text { Dental } \\
\text { Office } \\
\text { Instruction }\end{array}$} & \multicolumn{3}{|c|}{ Clinical Screening } & \multirow[t]{2}{*}{ Total, N (\%) } & \multirow{2}{*}{$\begin{array}{c}\text { P- } \\
\text { Value }\end{array}$} \\
\hline & Good, N (\%) & Fair, N (\%) & Poor, N (\%) & & \\
\hline No & $3,922(66.60)$ & $1,481(25.15)$ & $486(8.25)$ & $5,889(20.90)$ & 0.000 \\
\hline Yes & $16,646(74.7)$ & $4,480(20.10)$ & $1,159(5.20)$ & $22,285(79.10)$ & \\
\hline Total & $20,568(73.00)$ & $5,961(21.16)$ & $1,645(5.84)$ & $28,174(100.00)$ & \\
\hline
\end{tabular}

Figure 13: Relation Between Clinical Screening and Oral Health Education from a Dental Office

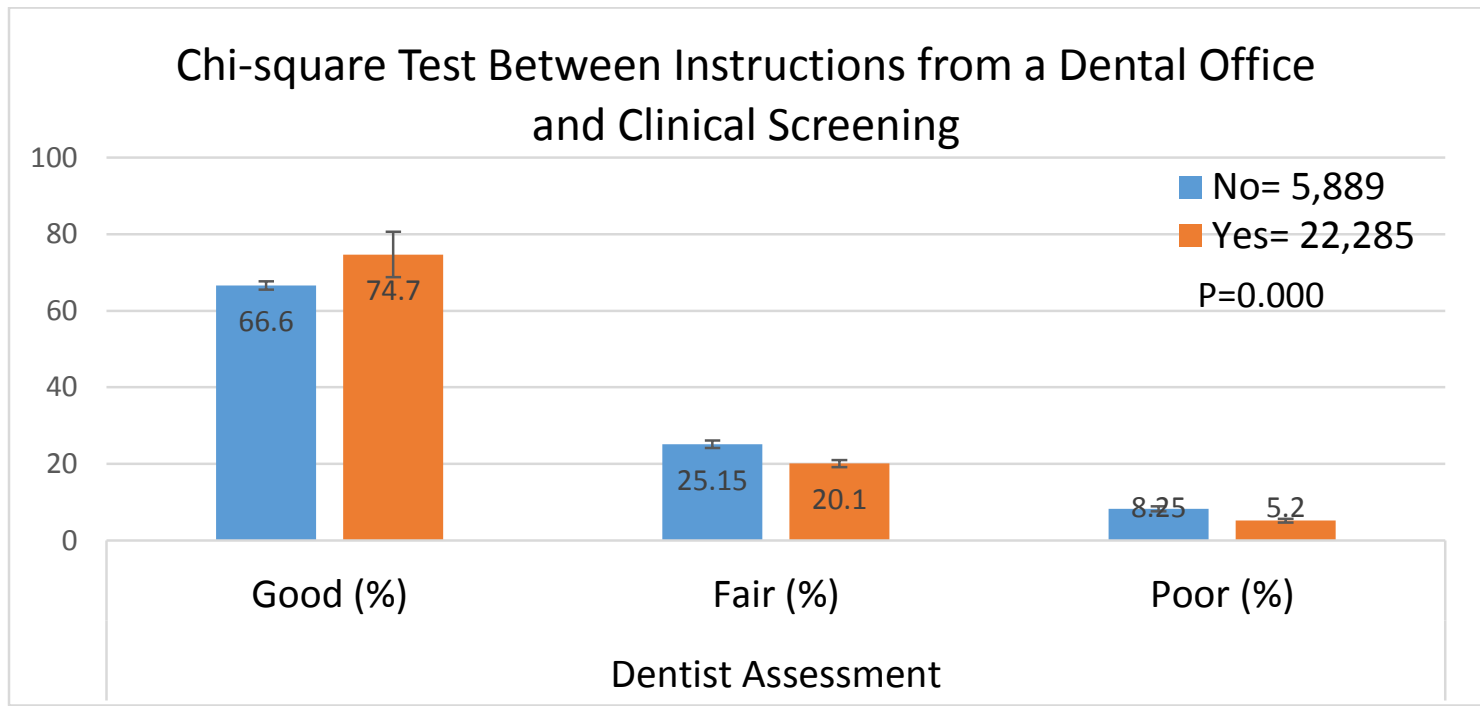


Table $14, \mathrm{P}=0.243$, is above the significance level of 0.05 . This shows that dental health training from physician office is in weak association with Clinical Screening by licensed dental practitioner. Interestingly, as shown in Figure 14, the relationship is negative. Even though, the overall percentage for parents with physician office training is less, (9\%), one-tenth of them had poor clinical condition when evaluated by a licensed dentist and that is more than parents with no prior dental health training by two percent.

Table 14: Chi-square Test Between Physician Office Instructions and Clinical Screening

\begin{tabular}{|c|c|c|c|c|c|}
\hline \multirow{2}{*}{$\begin{array}{c}\text { Physician } \\
\text { office } \\
\text { Instruction }\end{array}$} & \multicolumn{3}{|c|}{ Clinical Screening } & Total, N (\%) & \multirow{2}{*}{$\begin{array}{c}\text { P- } \\
\text { Value }\end{array}$} \\
\cline { 2 - 5 } No & $3,922(66.60)$ & $1,481(25.15)$ & $486(8.25)$ & $5,889(90.98)$ & 0.2433 \\
\hline Yes & $379(64.90)$ & $145(24.83)$ & $60(10.27)$ & $584(9.02)$ & \\
\hline Total & $4,301(66.45)$ & $1,626(25.12)$ & $546(8.44)$ & $6,473(100.00)$ & \\
\hline
\end{tabular}

Figure 14: Relation Between Clinical Screening and Oral Health Education from a Physician Office

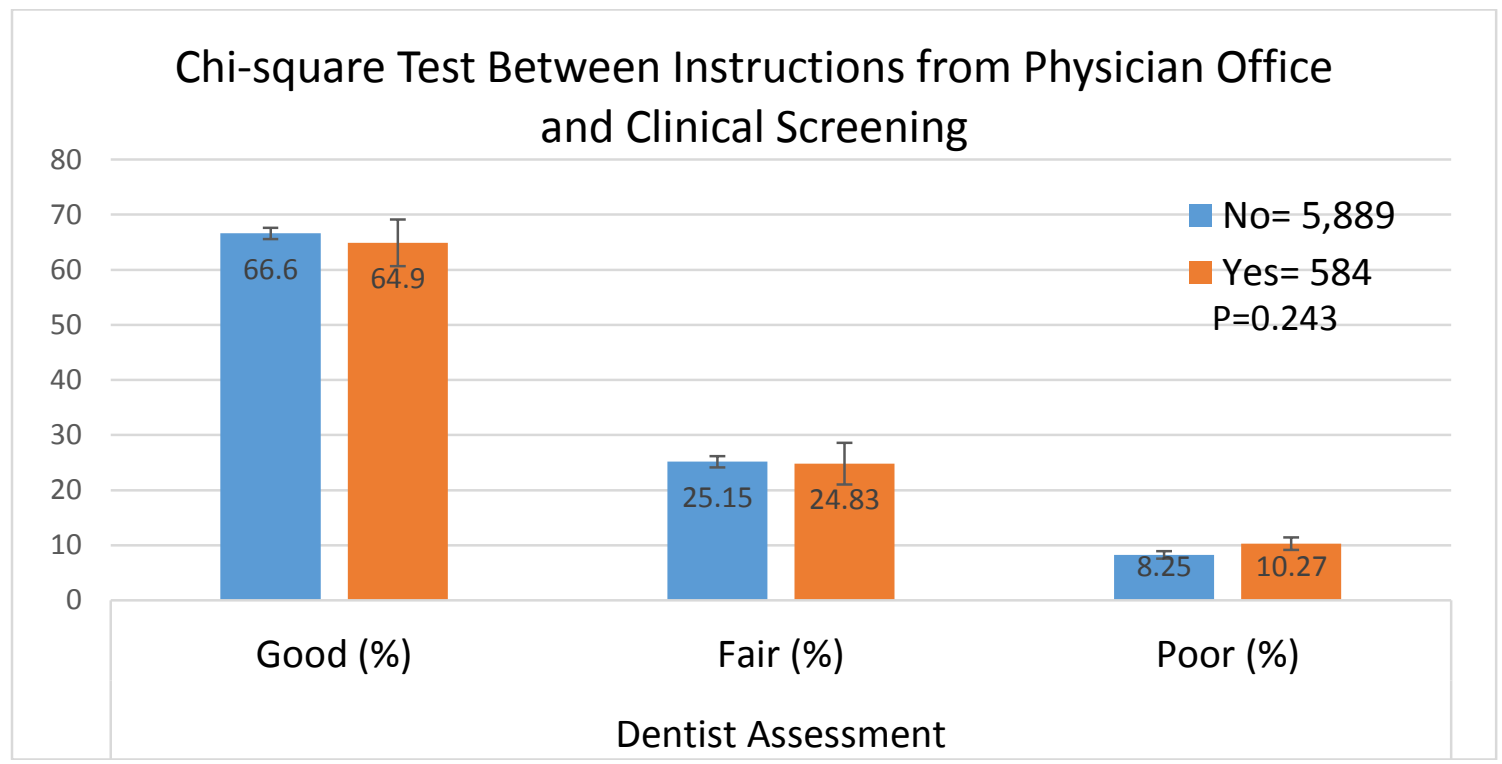


Table 15, $\mathrm{P}<0.0001$ is far below the significance level of 0.05 . This shows that dental health training from a health department is strongly associated with clinical screening done by licensed dental practitioner. However, as seen in Figure 15, the association is negative. Similar to physician office education, health department training showed $11 \%$ more parenteral assessments of 'fair' and 'poor' clinical condition, which is three percent more than that of physician office. Compared to no oral health training, health department training showed a strong negative influence on a child's actual oral condition. The narrow interval of standard error bars increase the reliability of our findings.

Table 15: Chi-square Test Between Health Department Instructions and Clinical Screening

\begin{tabular}{|c|c|c|c|c|c|}
\hline \multirow{2}{*}{$\begin{array}{c}\text { Health } \\
\text { Department } \\
\text { Instruction }\end{array}$} & \multicolumn{3}{|c|}{ Clinical Screening } & Total, N (\%) & \multirow{2}{*}{$\begin{array}{c}\text { P- } \\
\text { Value }\end{array}$} \\
\cline { 2 - 5 } & Good, N (\%) & Fair, N (\%) & $\begin{array}{c}\text { Poor, N } \\
(\%)\end{array}$ & \\
\hline No & $3,922(66.6)$ & $1,481(25.15)$ & $486(8.25)$ & $5,889(94.31)$ & $<0.0001$ \\
\hline Yes & $197(55.49)$ & $117(32.96)$ & $41(11.55)$ & $355(5.69)$ & \\
\cline { 1 - 4 } Total & $4,119(65.97)$ & $1,598(25.59)$ & $527(8.44)$ & 6,244 & \\
& & & & & \\
\hline
\end{tabular}

Figure 15: Relation Between Clinical Screening and Oral Health Education from a Health Department

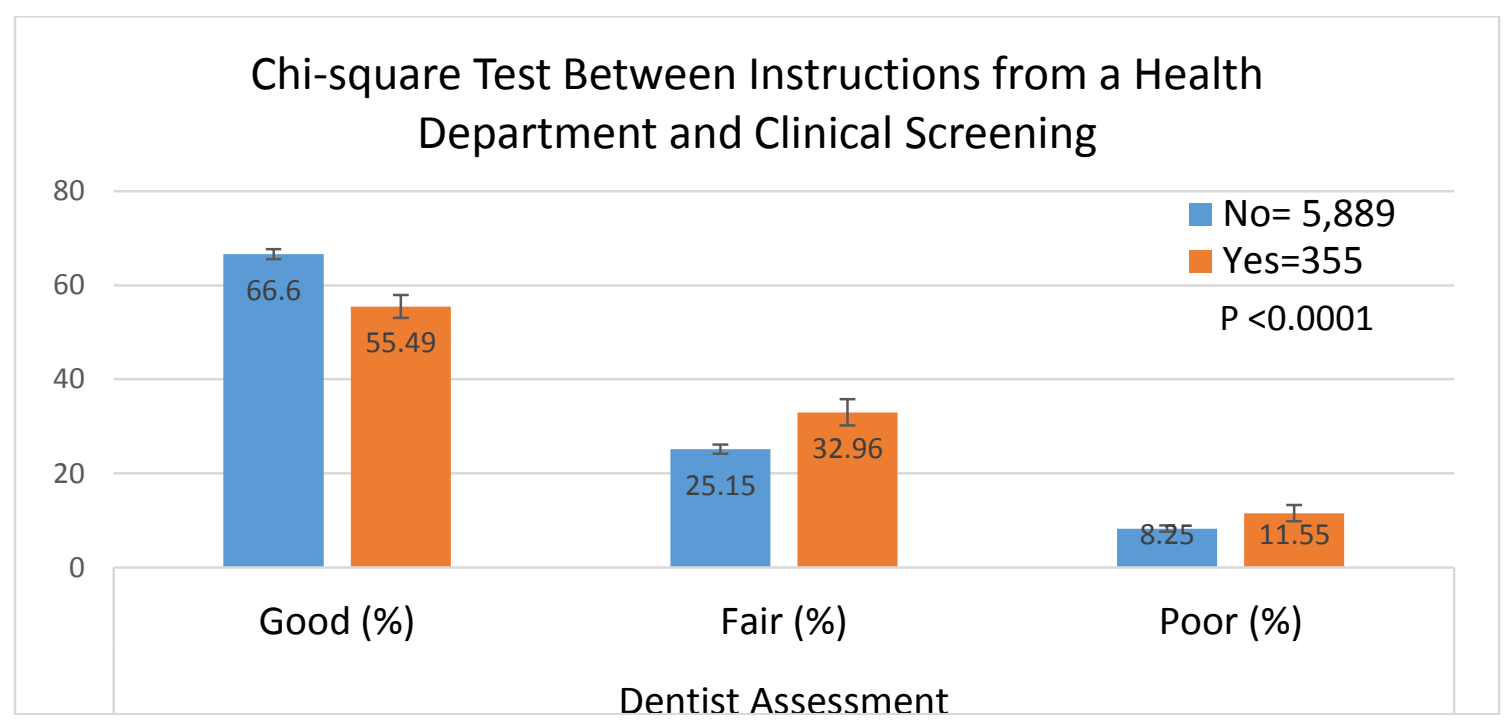


On Table 16, $\mathrm{P}<0.0001$ is far below the significance level of 0.05 . This shows that dental health training received from 'other' providers is significantly associated with clinical screening done by licensed dental practitioner. Compared with dental office, physician office and health department educated parents, oral health education from other provider sources showed the highest percentage of 'poor' $(12.2 \%)$ clinical condition of all of the children participated in our study. Figure 16 supports our findings graphically. Lack of dental health training showed a high rate of 'good' clinical condition over the clinical conditions of children with parent education from other sources by six percent. With reasons unexplained, the influence needs to be explored further.

Table 16: Chi-square Test Between 'Other' Provider Instructions and Clinical Screening

\begin{tabular}{|c|c|c|c|c|c|}
\hline \multirow{2}{*}{$\begin{array}{c}\text { Other } \\
\text { Instruct }\end{array}$} & \multicolumn{3}{|c|}{ Clinical Screening } & Total, N (\%) & P- \\
\cline { 2 - 4 } & Good, N (\%) & Fair, N (\%) & Poor, N (\%) & & Value \\
\hline No & $3922(66.6)$ & $1481(25.15)$ & $486(8.25)$ & $5889(87.84)$ & $<0.000$ \\
\cline { 1 - 4 } Yes & $501(61.47)$ & $207(25.40)$ & $107(13.13)$ & $815(12.16)$ & 1 \\
\hline Total & $4423(65.98)$ & $1688(25.18)$ & $593(8.85)$ & $6704(100.00)$ & \\
\hline
\end{tabular}

Figure 16: Relation Between Clinical Screening and Oral Health Education from 'Other' Provider Sources

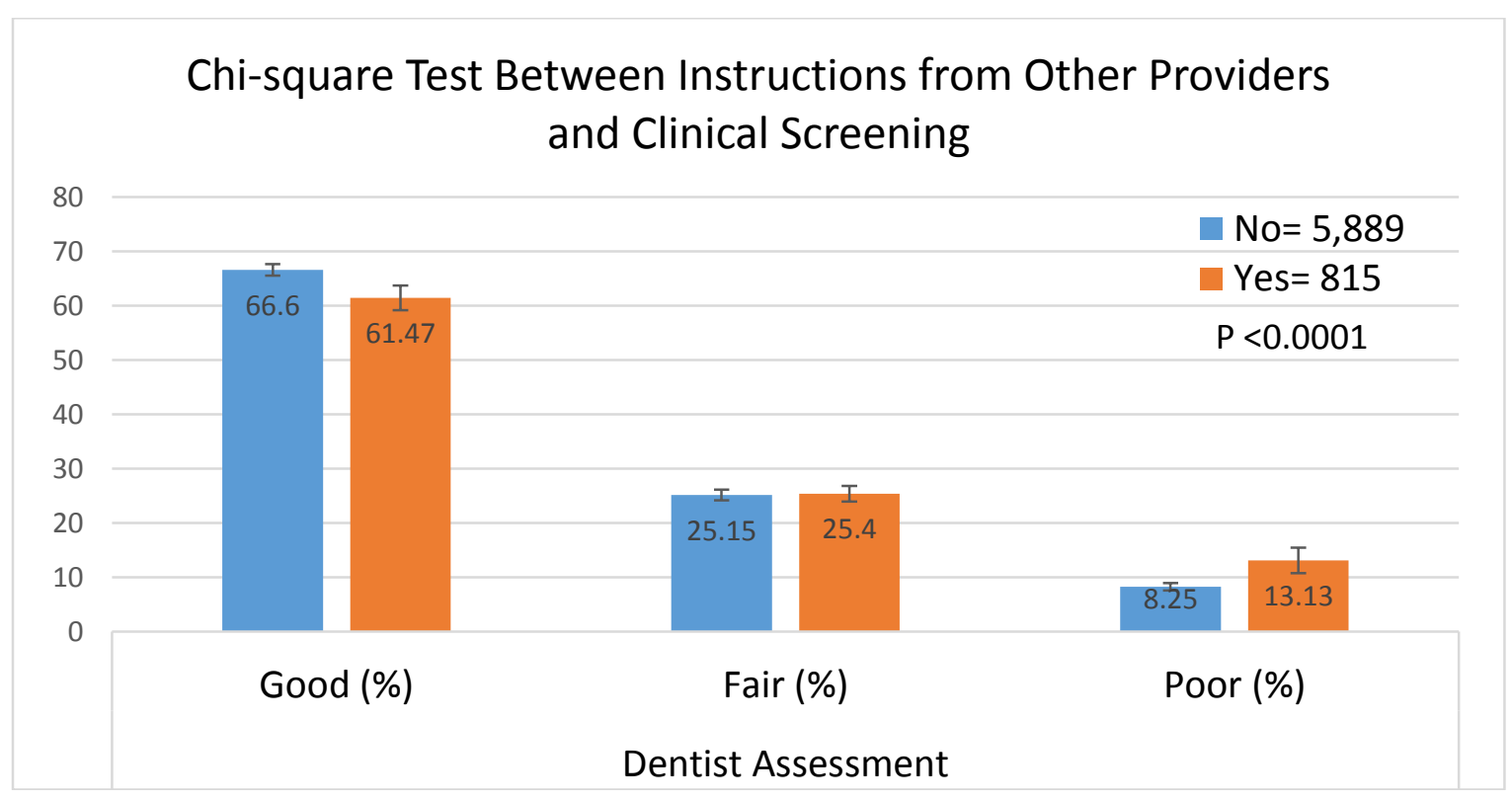


Table 17 and Figure 17 compared the parental assessments with actual clinical condition of the child's teeth. Parents overrated 'good' and 'fair' condition of their child's teeth by $0.5 \%$ and $2 \%$ respectively. There was an underestimation of 'poor' dental condition significantly by $3.1 \%$. Standard errors calculated at $95 \%$ Confidence Interval increased the validity and reliability of our findings. This further reflects the discrepancy between parental assessments based on individual perceptions and the actual clinical condition of the child's teeth.

Table 17: Chi-square Test Between Parental Assessment and Clinical Screening

\begin{tabular}{|c|c|c|c|c|c|}
\hline \multirow{2}{*}{$\begin{array}{c}\text { Parental } \\
\text { Assessmen } \\
\text { t }\end{array}$} & \multicolumn{3}{|c|}{ Clinical Screening } & Total, N (\%) & P- \\
\cline { 2 - 5 } & Good, N (\%) & Fair, N (\%) & Poor, N (\%) & & \\
\hline Good & $19,051(77.90)$ & $4,571(18.69)$ & $835(3.41)$ & $24,457(73.15)$ & $<0.000$ \\
\hline Fair & $4,740(59.80)$ & $2,260(28.51)$ & $926(11.68)$ & $7,926(23.71)$ & 1 \\
\hline Poor & $477(45.47)$ & $302(28.79)$ & $270(25.74)$ & $1,049(3.14)$ & \\
\hline Total & $24,268(72.59)$ & $7,133(21.34)$ & $2,031(6.08)$ & $33,432(100.00)$ & \\
\hline
\end{tabular}

Figure 17: Comparison of Parental Assessment with Clinical Screening Results

\section{Chi-square Test Beween Parental Assessment and Clinical Screening Results}

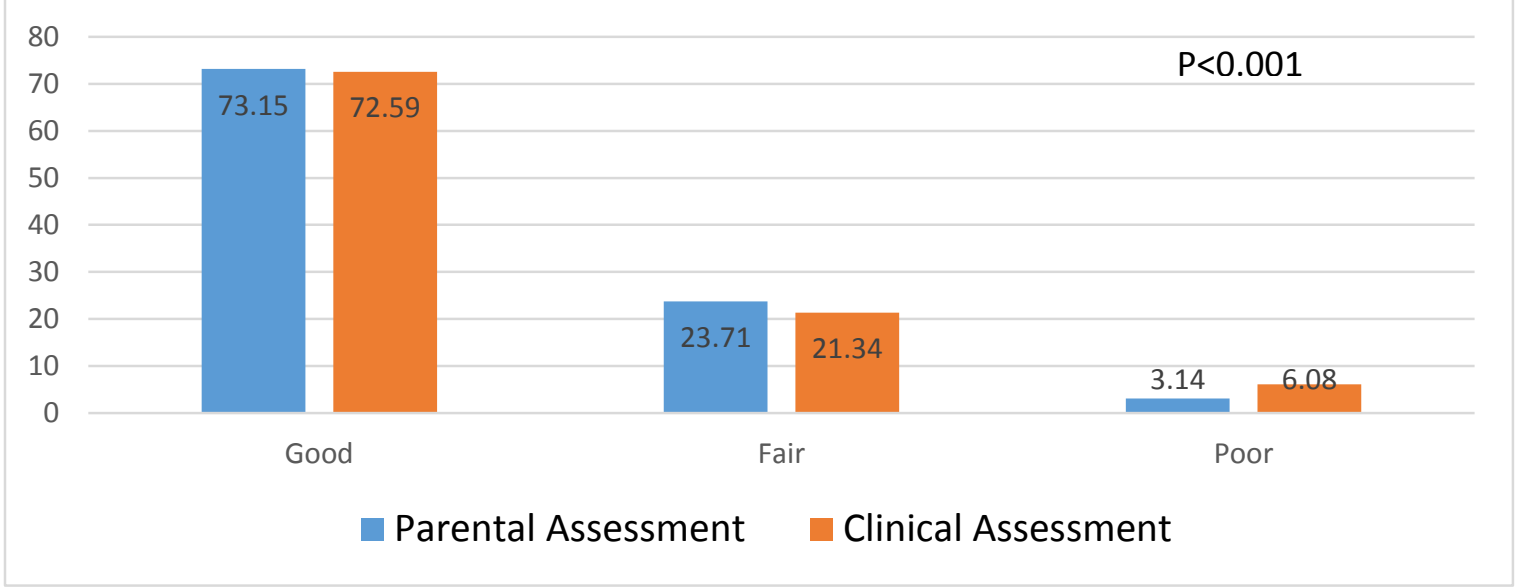


Tables 18 through 22 demonstrate the comparisons of clinical screening results with parental assessments, recorded separately for the four different providers. Based on P-value of $<0.0001$, all associations were strongly significant. With an aim to identify the provider with highest positive influence, we did a one-on-one comparison by charting a bar graph with total correct and total wrong numbers for four different providers along with a bar designating the lack of dental education with very interesting results. Trend analysis was performed for all four provider groups and standard errors were calculated (Appendix A).

Table 18: Chi-square Test Between Parental Assessment Based on Instructions from a Dental Office and Clinical Screening Results

\begin{tabular}{|c|c|c|c|c|c|}
\hline \multirow{2}{*}{$\begin{array}{c}\text { Parental } \\
\text { Assess }\end{array}$} & \multicolumn{2}{|c|}{ Clinical Screening Results } & Total, N (\%) & \multirow{2}{*}{$\begin{array}{c}\text { P- } \\
\text { Value }\end{array}$} \\
\cline { 2 - 4 } Good & Good, N (\%) & Fair, N (\%) & Poor, N (\%) & & $<0.00$ \\
\hline Fair & $4,005(180(57.43)$ & $3,846(13.65)$ & $683(2.42)$ & $20,709(73.50)$ & 01 \\
\hline Poor & $383(1.36)$ & $1,879(6.67)$ & $760(2.70)$ & $6,644(23.58)$ & \\
\hline Total & $20,568(73.00)$ & $5,961(21.16)$ & $1,645(5.84)$ & $28,174(100.00)$ & \\
\hline
\end{tabular}

Table 19: Chi-square Test Between Parental Assessment Based on Instructions from a Physician Office and Clinical Screening Results

\begin{tabular}{|c|c|c|c|c|c|}
\hline \multirow{2}{*}{$\begin{array}{c}\text { Parental } \\
\text { Assess }\end{array}$} & \multicolumn{3}{|c|}{ Clinical Screening Results } & Total, N (\%) & P- \\
\cline { 2 - 4 } & Good, N (\%) & Fair, N (\%) & Poor, N (\%) & & Value \\
\hline Good & $3,120(48.20)$ & $958(14.80)$ & $208(3.21)$ & $4,286(66.21)$ & $<0.000$ \\
\hline Fair & $1,039(16.05)$ & $566(8.74)$ & $249(3.85)$ & $1,854(28.64)$ & 1 \\
\hline Poor & $142(2.19)$ & $102(1.58)$ & $89(1.37)$ & $333(5.14)$ & \\
\hline Total & $4,301(66.45)$ & $1,626(25.12)$ & $546(8.44)$ & $6,473(100.00)$ & \\
\hline
\end{tabular}

Table 20: Chi-square Test Between Parental Assessment Based on Instructions from a Health Department and Clinical Screening Results

\begin{tabular}{|c|c|c|c|c|c|}
\hline \multirow{2}{*}{$\begin{array}{c}\text { Parental } \\
\text { Assessment }\end{array}$} & \multicolumn{3}{|c|}{ Clinical Screening Results } & \multirow[t]{2}{*}{ Total, N (\%) } & \multirow{2}{*}{$\begin{array}{c}\text { P- } \\
\text { Value }\end{array}$} \\
\hline & Good, N (\%) & Fair, N (\%) & Poor, N (\%) & & \\
\hline Good & $2,979(47.71)$ & $925(14.81)$ & $199(3.19)$ & $4,103(65.71)$ & \multirow[t]{3}{*}{$<0.0001$} \\
\hline Fair & $997(15.97)$ & $564(9.03)$ & $239(3.83)$ & $1,800(28.83)$ & \\
\hline Poor & $143(2.29)$ & $109(1.75)$ & $89(1.43)$ & $341(5.46)$ & \\
\hline
\end{tabular}


Total

\begin{tabular}{|c|c|}
$4,119(65.97)$ & $\begin{array}{c}1,598 \\
(25.59)\end{array}$ \\
\hline
\end{tabular}

\begin{tabular}{|l|l|}
\hline $527(8.44)$ & $6,244(100.00)$
\end{tabular}

Table 21: Chi-square Test Between Parental Assessment Based on Instructions from Other Providers and Clinical Screening Results

\begin{tabular}{|c|c|c|c|c|c|}
\hline \multirow{2}{*}{$\begin{array}{c}\text { Parental } \\
\text { Assess }\end{array}$} & \multicolumn{3}{|c|}{ Clinical Screening Results } & \multirow[t]{2}{*}{ Total $(\%)$} & \multirow{2}{*}{$\begin{array}{c}\text { P- } \\
\text { Value }\end{array}$} \\
\hline & Good, N (\%) & Fair, N (\%) & Poor, N (\%) & & \\
\hline Good & $3,196(47.67)$ & $964(14.38)$ & $229(3.42)$ & $4,389(65.47)$ & \multirow{4}{*}{$\begin{array}{c}<0.000 \\
1\end{array}$} \\
\hline Fair & $1,068(15.93)$ & $614(9.16)$ & $265(3.95)$ & $1,947(29.04)$ & \\
\hline Poor & $159(2.37)$ & $110(1.64)$ & $99(1.48)$ & $368(5.49)$ & \\
\hline Total & $4,423(65.98)$ & $1,688(25.18)$ & $593(8.85)$ & $6,704(100.00)$ & \\
\hline
\end{tabular}

Table 22: Chi-square Test Between Parental Assessment Based on 'NO' Instructions Received from Any of the Providers and Clinical Screening Results

\begin{tabular}{|c|c|c|c|c|c|}
\hline \multirow{2}{*}{$\begin{array}{c}\text { Parental } \\
\text { Assess }\end{array}$} & \multicolumn{3}{|c|}{ Clinical Screening Results } & Total, N (\%) & P- \\
\cline { 2 - 4 } & Good, N (\%) & Fair, N (\%) & Poor, N (\%) & & Value \\
\hline Good & $2,850(48.40)$ & $863(14.65)$ & $185(3.14)$ & $3,898(66.19)$ & $<0.000$ \\
\hline Fair & $937(15.91)$ & $523(8.88)$ & $223(3.79)$ & $1,683(28.58)$ & 1 \\
\hline Poor & $135(2.29)$ & $95(1.61)$ & $78(1.32)$ & $308(5.23)$ & \\
\hline Total & $3,922(66.60)$ & $1,481(25.15)$ & $486(8.25)$ & $5,889(100.00)$ & \\
\hline
\end{tabular}

Figure 18, total correct parental assessment is high with instructions from a dental office. Which means that instructions received from a dental office are significantly influencing correct parental assessment when compared to other providers! Even though, the difference is only $6 \%$ (dental providers $=64 \%$ and other providers $58 \%$ ), the influence of other providers is similar to no form of dental health education at all. 
Figure 18: Comparison of Parental Assessment with Clinician Screening Results Based on Oral Health Training from Different Providers - Total Correct Percentage

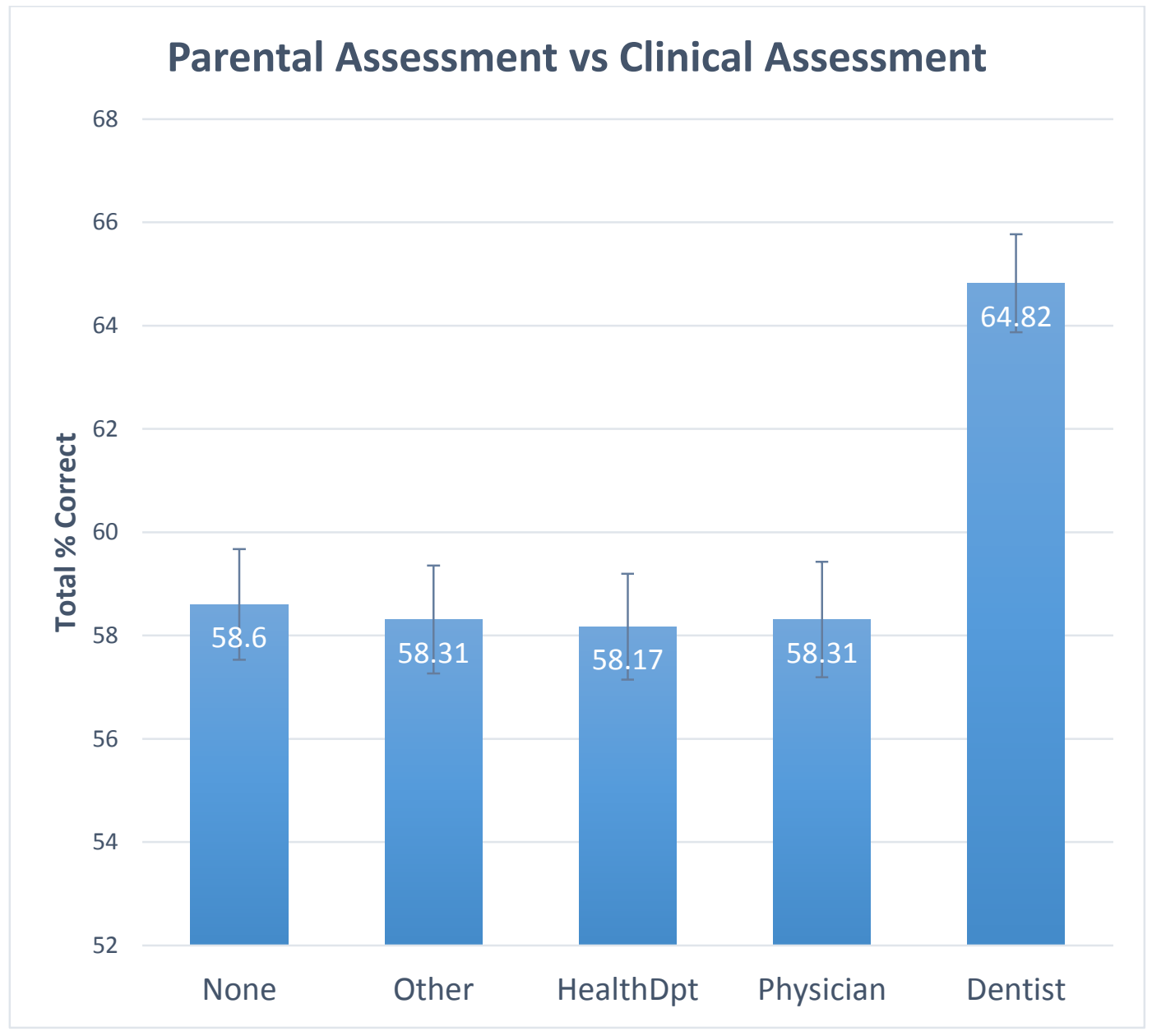

Correspondingly Figure 18 demonstrates the number of correct parenteral assessments and total wrong parenteral assessments is found on Figure 19. There was approximately a $6 \%$ discrepancy between dental office oral health education physician office, health department and other provider education. With the exception of other providers, we are $95 \%$ confident that the error is less than $2 \%$ (1.96) in representation of our population mean. 
Figure 19: Comparison of Parental Assessment with Clinician Screening Results Based on Oral Health Training from Different Providers - Total Wrong Percentage

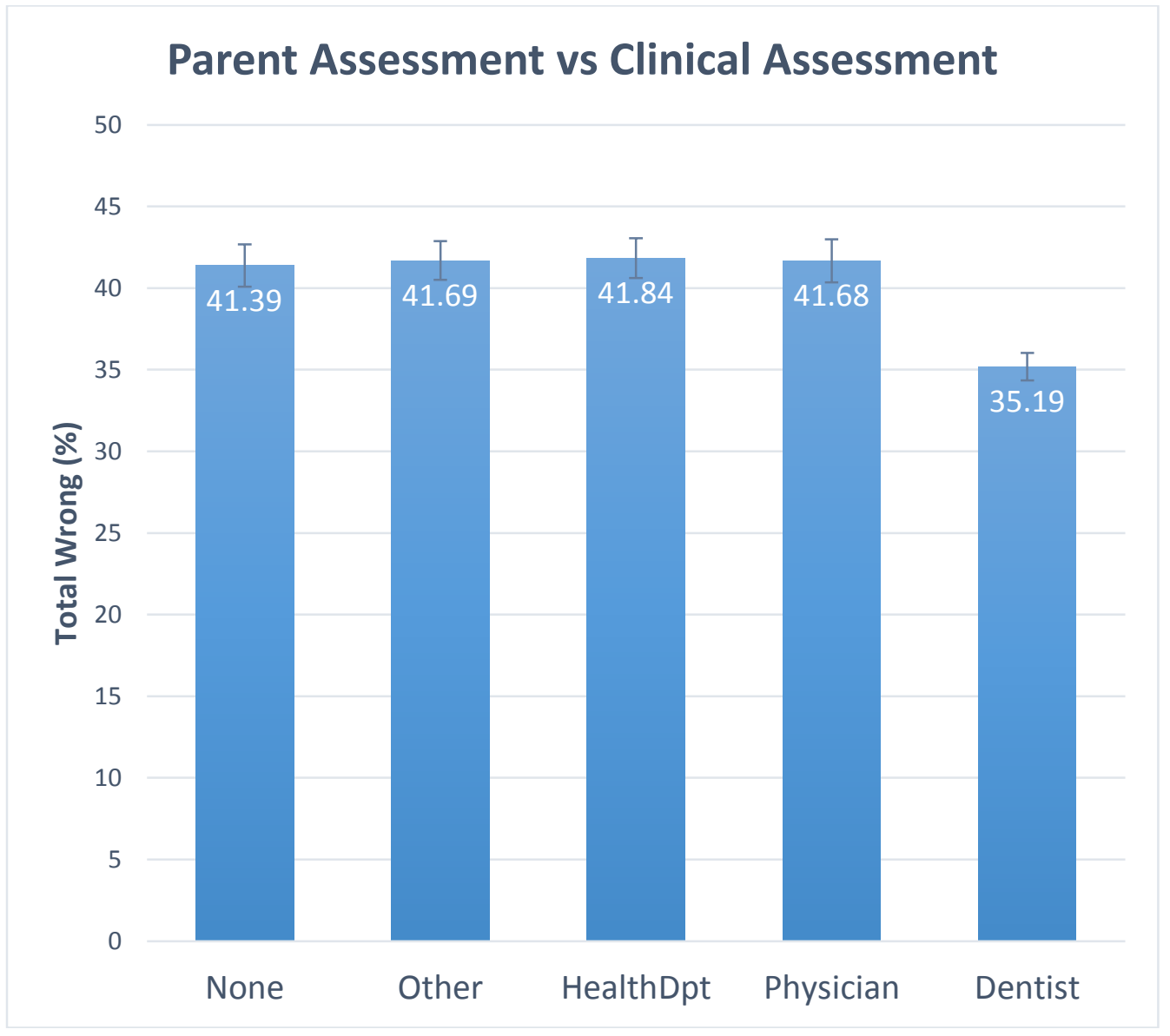


The results from multivariate logistic regression model were presented in Tables 23 and 24. Initially, all demographics and variables representing clinical findings were included in our regression model. Based on the p-value of $<0.05$, we eliminated nonsignificant variables. The models were highly significant with a $\mathrm{p}<0.05$ for all the predictors as shown in the tables. The narrow 95\% confidence intervals and corresponding standard errors increase the reliability and validity of our significant model. Our final model included carious quadrants, carious lesions, malocclusion, poor oral hygiene, molar sealants, presence of plaque, insurance coverage, dental office visits for regular check-ups and oral health education received from a dental office. Instructions received from a physician's office, health department and other providers demonstrated an insignificant association in the final model and hence were removed.

Table 23: Multivariate Logistic Regression Model (Base Level=0, Good): Good vs Fair Parenteral Assessments

\begin{tabular}{|l|c|c|c|c|c|c|c|}
\hline \multicolumn{1}{|c|}{ Variable } & Coefficient & s.e. & Z-Test & $\begin{array}{c}\text { P- } \\
\text { Value }\end{array}$ & $\begin{array}{c}\text { Odds } \\
\text { Ratio }\end{array}$ & $\begin{array}{c}\text { Lower } \\
\text { CI }\end{array}$ & $\begin{array}{c}\text { Upper } \\
\text { CI }\end{array}$ \\
\hline Intercept & -0.760 & 0.040 & 363.581 & 0.000 & & & \\
\hline $\begin{array}{l}\text { Instructor } \\
\text { Dental } \\
\text { Office }\end{array}$ & -0.108 & 0.028 & 14.3222 & 0.000 & 0.898 & 0.849 & 0.949 \\
\hline Visit Reason & -0.567 & 0.029 & 387.534 & 0.000 & 0.567 & 0.536 & 0.600 \\
\hline Insurance & -0.252 & 0.034 & 55.550 & 0.000 & 0.777 & 0.728 & 0.831 \\
\hline $\begin{array}{l}\text { Poor Oral } \\
\text { Hygiene }\end{array}$ & 0.170 & 0.077 & 4.880 & 0.027 & 1.185 & 1.019 & 1.378 \\
\hline Plaque & -0.600 & 0.067 & 81.080 & 0.000 & 0.549 & 0.482 & 0.625 \\
\hline Malocclusion & 0.390 & 0.029 & 186.516 & 0.000 & 1.476 & 1.396 & 1.561 \\
\hline Cavities & 0.402 & 0.064 & 39.547 & 0.000 & 1.495 & 1.319 & 1.694 \\
\hline $\begin{array}{l}\text { Sealants } \\
\text { molars }\end{array}$ & -0.241 & 0.033 & 54.424 & 0.000 & 0.785 & 0.737 & 0.837 \\
\hline $\begin{array}{l}\text { Carious } \\
\text { Quadrants }\end{array}$ & 0.413 & 0.065 & 40.326 & 0.000 & 1.512 & 1.331 & 1.718 \\
\hline
\end{tabular}


Table 24: Multivariate Logistic Regression Model (Base Level=0, Good): Good vs Poor Parenteral Assessments

\begin{tabular}{|l|c|c|c|c|c|c|c|}
\hline \multicolumn{1}{|c|}{ Variable } & Coefficient & s.e. & Z-Test & $\begin{array}{c}\text { P- } \\
\text { Value }\end{array}$ & $\begin{array}{c}\text { Odds } \\
\text { Ratio }\end{array}$ & $\begin{array}{c}\text { Lower } \\
\text { CI }\end{array}$ & $\begin{array}{c}\text { Upper } \\
\text { CI }\end{array}$ \\
\hline Intercept & -2.206 & 0.082 & 718.948 & 0.000 & & & \\
\hline $\begin{array}{l}\text { Instructor } \\
\text { Dental } \\
\text { Office }\end{array}$ & -0.465 & 0.067 & 48.997 & 0.000 & 0.628 & 0.551 & 0.715 \\
\hline Visit Reason & -1.166 & 0.068 & 291.698 & 0.000 & 0.312 & 0.273 & 0.356 \\
\hline Insurance & -0.668 & 0.071 & 89.810 & 0.000 & 0.513 & 0.446 & 0.589 \\
\hline $\begin{array}{l}\text { Poor Oral } \\
\text { Hygiene }\end{array}$ & 0.599 & 0.151 & 15.640 & 0.000 & 1.820 & 1.353 & 2.448 \\
\hline Plaque & -0.611 & 0.145 & 17.828 & 0.000 & 0.543 & 0.409 & 0.721 \\
\hline Malocclusion & 0.419 & 0.069 & 37.379 & 0.000 & 1.521 & 1.330 & 1.740 \\
\hline Cavities & 0.611 & 0.148 & 17.136 & 0.000 & 1.843 & 1.380 & 2.461 \\
\hline $\begin{array}{l}\text { Sealants } \\
\text { molars }\end{array}$ & -0.229 & 0.087 & 6.933 & 0.008 & 0.795 & 0.671 & 0.943 \\
\hline $\begin{array}{l}\text { Carious } \\
\text { Quadrants }\end{array}$ & 0.588 & 0.147 & 16.036 & 0.000 & 1.800 & 1.350 & 2.401 \\
\hline
\end{tabular}

Table 25: Classification Table for Multiple Logistic Regression Modeling

\begin{tabular}{|l|r|r|r|r|r|}
\hline \multirow{2}{*}{ Observed } & \multicolumn{4}{|c|}{ Predicted } & \multicolumn{1}{c|}{$\begin{array}{c}\text { Total } \\
\text { (Actual) }\end{array}$} \\
\cline { 2 - 5 } & Good & \multicolumn{1}{c|}{ Fair } & \multicolumn{1}{c|}{ Poor } & $\begin{array}{c}\text { Percent } \\
\text { Correct }\end{array}$ & \\
\hline Good & $\mathbf{2 3 , 9 8 5}$ & 472 & 0 & 98.1 & 24,457 \\
\hline Fair & 7,405 & $\mathbf{5 2 1}$ & 0 & 6.6 & 7,926 \\
\hline Poor & 890 & 159 & $\mathbf{0}$ & 0.0 & 1,049 \\
\hline Total & 32,243 & 1,189 & 0 & 100.0 & 33,432 \\
\hline $\begin{array}{l}\text { Overall } \\
\text { Percentage }\end{array}$ & $96.6 \%$ & $3.4 \%$ & $0.0 \%$ & $\mathbf{7 3 . 3 \%}$ & $\mathbf{1 0 0 . 0 0 \%}$ \\
\hline
\end{tabular}

Table 25 represents the classification table for multivariate logistic regression. A total of 24,506 subjects out of 33,432 were correctly classified. This represents $73.3 \%$ of our whole population observations were correctly classified. Therefore we can say that the multivariate logistic regression was well performed on this data. 
Figures 20 and 21 show the odds of fair and poor parental assessments over good parental assessment. Odds ratio of greater than one indicate that presence of carious quadrants, carious lesions, malocclusion and poor oral hygiene increase the number of fair and poor parental assessments by one unit over good parental assessment. Odds ratio $<1$ for molar sealants, plaque, insurance, dental office visits for regular check-ups and oral health education received from a dental office decrease fair and poor parental assessments or increase good parental assessments. In particular, oral health education from a dental office decreases fair parental assessment by $90 \%$ and poor parental assessment by $63 \%$, which further supports our findings from chi-square analysis.

Figure 20: Representation of Odds Ratio for 'Fair' Parental Assessments

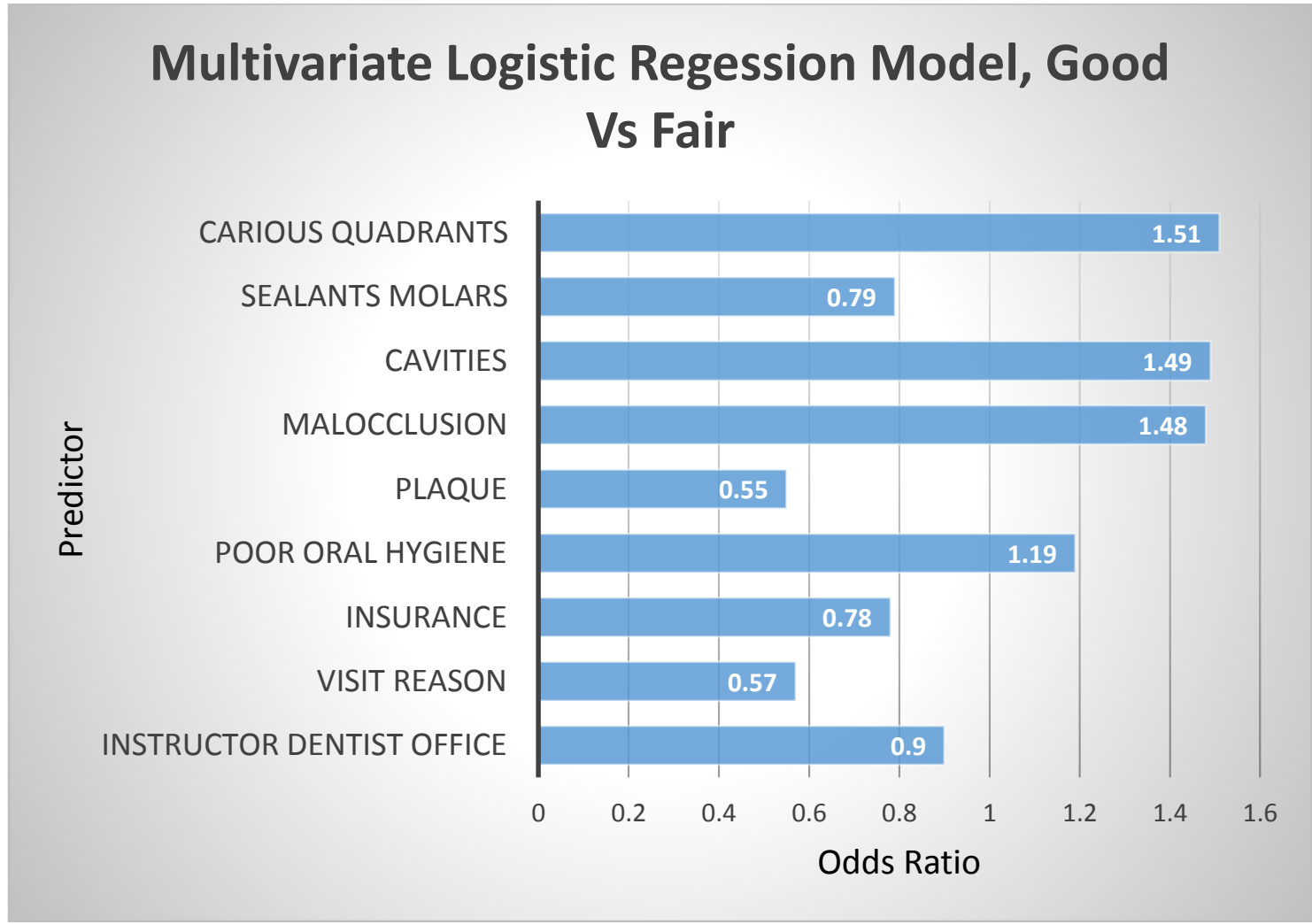


Figure 21: Representation of Odds Ratio for 'Poor' Parental Assessments

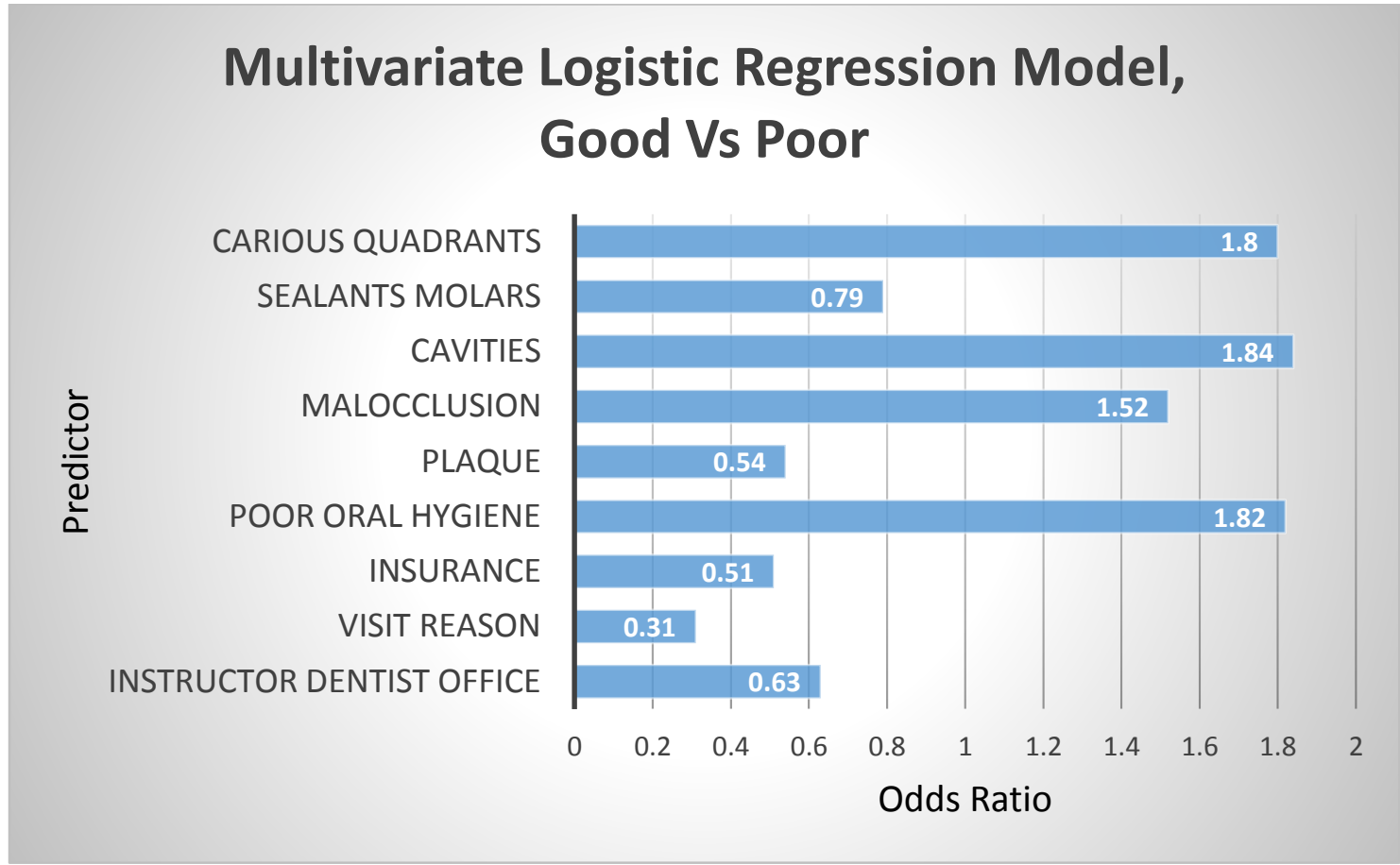

Table 26 (Appendix A) and Figure 22 compare caries rate in terms of the total number of cavities decreased by $7 \%$ over the past 12 years $(2003=31 \%, 2014=24.1 \%)$. Further, caries rate was low in Bullitt County compared to Jefferson County. Even though Bullitt county had less enrollment overall, they represent our patient population being exposed to Smile KY annual screenings annually. To simplify, smile KY screenings resulted in a reduction of caries rate in Bullitt County by 3.8\%. A corresponding increase in caries rate is observed in Jefferson County. 
Figure 22: Trend Analysis for Carious Lesions Over 11 years of Screening

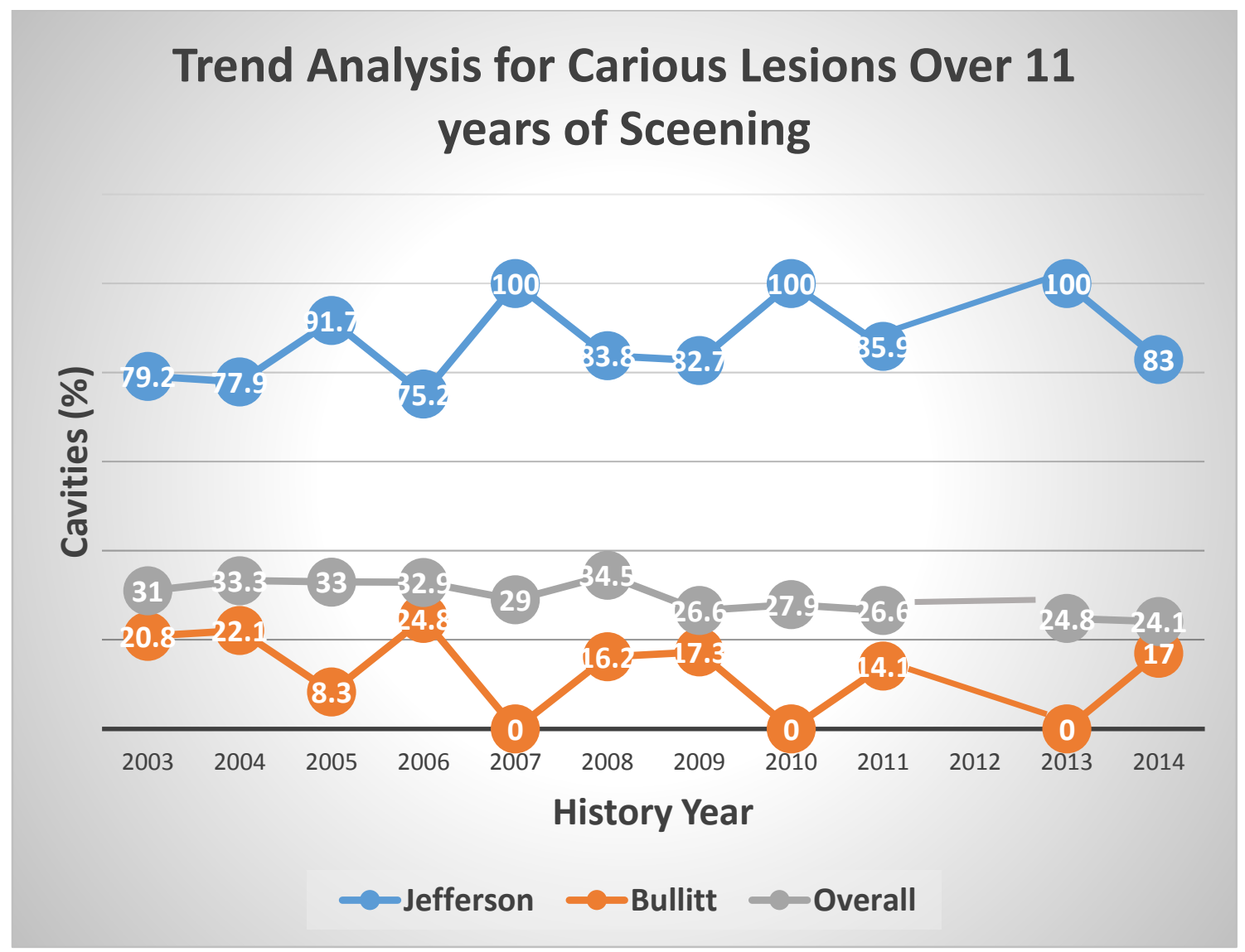

Table 27 and Figure 23 show that dental office visits are pretty consistent with minor fluctuations in the 12-years of our research period and more than $90 \%$ of the annual population screened. Encouragingly, the numbers are more than $95 \%$ from the 2013 outcomes. We expect the trend to continue to improve and reach $100 \%$ in the near future. Compared to Bullitt County, the Jefferson County population had a higher number of office visits over the 12 year history of Smile KY!. 
Figure 23: Trend Analysis for Dental Office Visits Over the 11 Years of Smile KY!

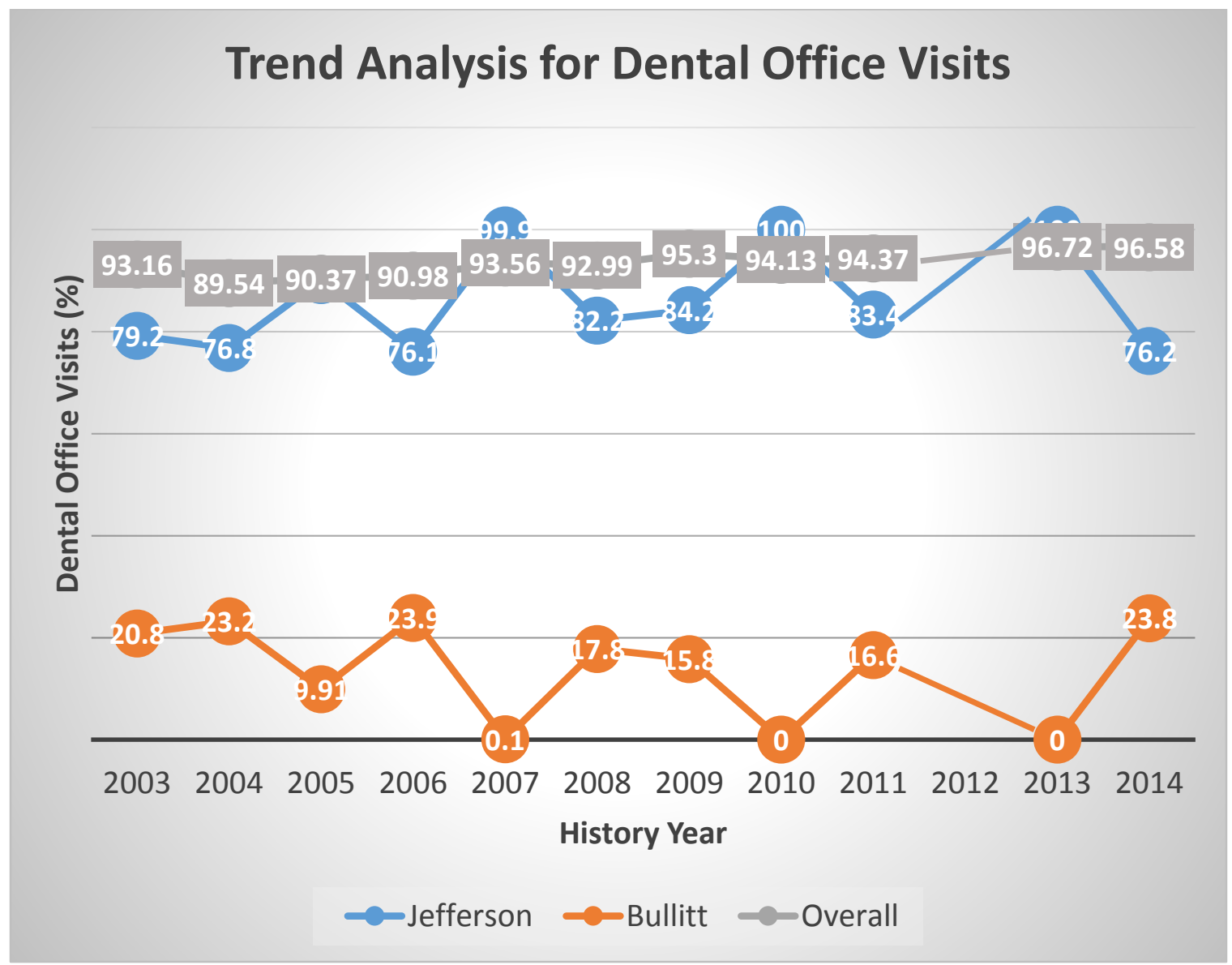

Table 28 (Appendix A) and Figure 24 show a declining trend in insurance coverage from 2003 to 2005 . Insurance coverage showed a steady increase till 2010. In 2009 dental insurance coverage through Medicaid, KCHIP or private insurance was above $80 \%$. There was a marked decrease in the number of children without insurance, which can be seen as the result of the Affordable Care Act. Overall, more than $75 \%$ of Smile KY! children had dental insurance coverage over the past 11 years. 
Figure 24: Trend Analysis for Dental Insurance Over the 11 Years History of Smile KY!

\section{Trend Analysis in Dental Insurance Over 11 years}

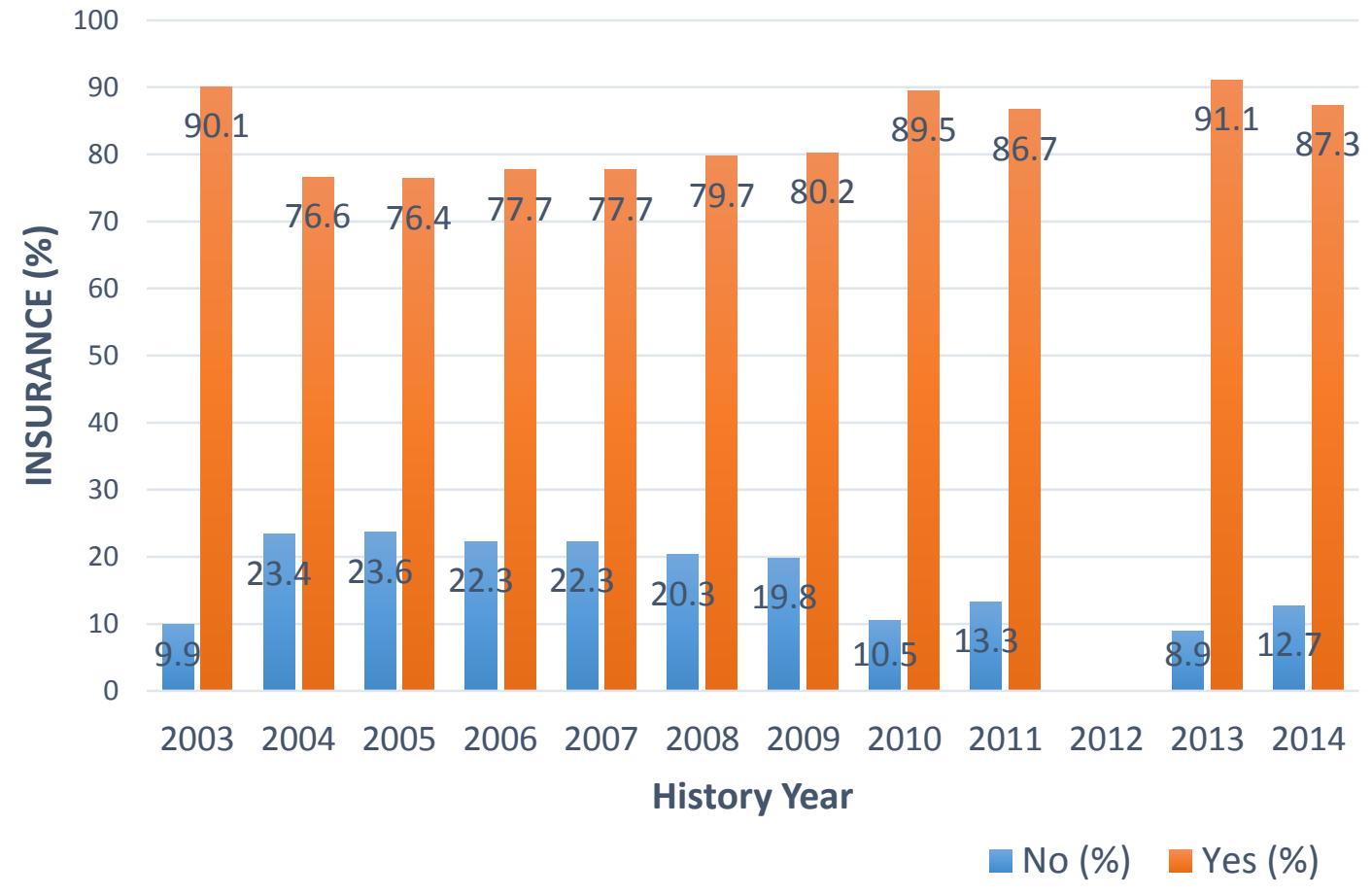


Children with poor oral health are twice as likely to have poor academic performance due to missed school days from dental pain. ${ }^{11}$ Dental pain and poor oral health in children and adolescents is associated with psychological disturbances affecting the child's self esteem and social development. ${ }^{9}$ Considering how poor oral health in early childhood and the importance of the parental role in their child's systemic and oral health, it seems that education for the parents can play a vital role in the prevention of future dental disease. ${ }^{43}$

Studies have shown that parental perceptions, attitudes, behavior, brushing skills, income level, educational level, ethnical background have a great deal of importance in determining a child's oral health status. ${ }^{\text {footnotes }}$ The effect of professionally delivered oral health instructions on parental perceptions has not yet been studied. A study comparing the influence of oral health education from different types of healthcare providers on parental assessment of their child's dental health is unknown. We have found that parental perceptions are dependent $(\mathrm{p}<0.0001)$ on the source of the professionally delivered oral hygiene instructions. A strong positive correlation was observed between oral health training and parental assessments with our cross-sectional study design, but cause-effect association was not established.

Most of the parents answering our survey $(\mathrm{n}=82 \%)$ received oral health education from one of the different healthcare providers, such as a dental office, a physician office, a health department or other source. The four possible providers were significantly $(\mathrm{p}<0.01)$ associated with the parental assessment of their child's oral health status. The association between oral health instruction for all four providers and parental assessment of the child's oral health was significant when validated by a clinical screening by a licensed dental professional. When the influence of the four different providers types were compared, the 
dental office education demonstrated the most influence $(\mathrm{p}<0.001)$ on appropriate parental assessments. A strong positive correlation was observed between dental office oral health education and parental assessment. In particular, dental office education was shown to have a more significantly positive influence in a good parental assessment of their child's dental condition.

A strong correlation was not found with "other" healthcare providers due to the small numbers, the influence was negative. To improve the oral health status of children in our state, government programs offer financial incentives to physicians for delivering preventive oral care. Physicians in Kentucky can get a reimbursement of $\$ 15$ for a single application of fluoride varnish twice a year on a children between the ages of 1 to 5 years. ${ }^{44}$ Oral health education with involvement of pediatric healthcare providers aids in dental disease prevention. ${ }^{39}$

In addition to oral health education, we considered other variables for a possible correlative influence on parental assessments. Our logistic regression model showed the significant influence of dental office visits for regular check-ups, carious quadrants, carious lesions, malocclusion, poor oral hygiene, molar sealants, plaque and insurance on parental assessment as secondary risk factors.

While $98.9 \%$ of our parents completing surveys said their children had prior dental visits either for an exam (13.8\%) or treatment (86.2\%), the 2001 Kentucky Children's Oral Health Survey ${ }^{8}$ found that $39 \%$ of the children between the ages of 5 to 9 years had never visited a dentist. With other factors involved, we observed a fairly consistent level of parents stating their children had dental care from 2001 to 2014. Children from our study 
showed a higher percentage of previous dental office visits when compared to the national average, which was $83 \%$ in $2013 .{ }^{45}$

When compared to other findings from a 2001 Kentucky Children's Oral Health Survey, $43 \%$ of the children surveyed had untreated caries. With the limitation of patient populations of different size and composition, the caries rate in 2014 for the Smile KY! screening visits was only $24 \%$. Early childhood caries was showed to limit the quality of life from the perspective of parents and children. ${ }^{46}$ Other factors contributing to high caries numbers in early childhood, include minority ethnicity, low socioeconomic status, low educational level of the mother and lack of insurance. ${ }^{47}$

Parents who took their children to regular dental check up appointments and return to get treatment $(\mathrm{OR}=0.79, \mathrm{P}<0.01)$ provided more good oral health assessments over fair and poor rating, which was also showed to increase oral health related quality of life. ${ }^{46}$ Only $3 \%$ of our parents surveyed rated their children's oral health as 'poor' dental condition, it has potential to cause the child's school attendance and academic performance. ${ }^{10}$ While malocclusion is an esthetic concern, it may motivate a parent to take their child to a dentist for reasons other than oral health reasons. ${ }^{48}$

Insurance is considered to be an important factor increasing access to oral healthcare. ${ }^{49}$ An exposure to healthcare providers would benefit parents by providing oral health education. US dental expenditure numbers for 2011 reveals that 16 billion dollars out of 64 billion dollars is funded by government insurance programs, ${ }^{50}$ Medicaid and CHIP. In 2008 it was found that $73 \%^{51}$ of the US population had dental insurance coverage, which is less than the $80 \%$ of our Smile KY! population. This shows a higher enrollment in insurance of our study participants compared to the national insurance with dental 
coverage. From 2010 to 2014 dental insurance coverage increased to $85 \%$ of the Smile KY! children. This is appears to be a positive outcome of the Affordable Care Act.

The Smile KY screenings from 2003-2014 showed a reduction in caries of $7 \%$. Other factors that could have contributed to the caries reduction, there was a $3.4 \%$ increase of dental office visits. With intermittent minor fluctuations, our study showed a progressive trend of increasing insurance coverage and in-office visits over the 11 years of data. A declining caries rate in the Smile KY! population provides evidence that prevention and intervention programs can have a profound impact on child oral health. 


\section{Strengths}

Our sample size is large with 33,432 participants, therefore our findings are applicable to general population. Further, our study is longitudinal with data from 2002 till 2014. Stratification of the Smile KY! screening data into two subsets (Bullitt County and Jefferson County) contributes to increased validity of our study findings.

\section{Limitations}

Our study has certain limitations similar to other survey designs. For data collection, we relied on information parents provided by completion of health history questionnaire. With it, like other survey designs, we assume we had recall bias from our study participants. In addition, precise indicators parents used to rate their child's dentition as good, fair/poor are unknown. Further, with the cross-sectional study design, we were able to find correlations between study variables but unable to draw conclusions with cause-effect relationship. 


\section{CONCLUSIONS}

Our research supported the concept that parental perceptions are correlated with professionally delivered oral hygiene instructions. In particular, dental office patient education proved to have a highly significant positive influence on 'good' parental assessment of a child's oral health.

A multidisciplinary approach of oral health education with involvement of different healthcare providers should be considered for dental disease prevention in early childhood as a measure to ensure healthy dental condition in adult life. 


\section{REFERENCES}

1. Mathu-Muju KR. Kentucky rates second in U.S. in poor oral health 2008; http://ukhealthcare.uky.edu/health-info/publications/advancesinsights/pediatrics/Kentucky-rates-second-in-US-in-poor-oral-health/.

2. Surdu S, Langelier M, Baker B, Wang S, Harun N, Krohl D. Oral Health in Kentucky. Rensselaer, NY: Center for Health Workforce Studies, School of Public Health, SUNY Albany;2016.

3. Bush HM, Dickens NE, Henry RG, et al. Oral health status of older adults in Kentucky: results from the Kentucky Elder Oral Health Survey. Spec Care Dentist 2010;30(5):185-192.

4. United Health Foundation. Dental visit, Kentucky. America's Healthcare Rankings 2016; http://www.americashealthrankings.org/KY/dental. Accessed 3/24/16.

5. Childress MT, Smith-Mello M. Kentucky's oral health poses challenges. Foresight. 2007;50:1-12.

6. Kandel EA, Richards JM, Binkley CJ. Childhood caries in the state of Kentucky, USA: a cross-sectional study. BMC Oral Health. 2012;5:12-38.

7. Dawkins E, Michimi A, Ellis-Griffith G, Peterson T, Carter D, English G. Dental caries among children visiting a mobile dental clinic in South Central Kentucky: a pooled cross-sectional study. BMC Oral Health. 2013;13(1):1-9.

8. Hardison JD, Cecil JC, Mullins MR, White JA, Manz M, Ferretti GA. The 2001 Kentucky Children's Oral Health Survey: Findings for Children Ages 24 to 59 months and Their Caregivers. Pediatr Dent. 2003;25(4):365-372.

9. Guarnizo-Herreño CC, Wehby GL. Children's Dental Health, School Performance, and Psychosocial Well-Being. J Pediatr. 2012;161(6).

10. Holt K, Barzel R. Oral Health and Learning: When Children's Oral Health Suffers, So Does Their Ability to Learn. 3rd ed. Washington, DC: National Maternal and Child Oral Health Resource Center; 2013.

11. Jackson SL, Vann WF, Kotch JB, Pahel BT, Lee JY. Impact of poor oral health on children's school attendance and performance. Am J Public Health. 2011;101(10):1900-1906.

12. Talekar BS, Rozier RG, Slade GD, Ennett ST. Parental perceptions of their preschool-aged children's oral health. J Am Dent Assoc 2005;136(3):364-372.

13. Poutanen R, Lahti S, Tolvanen M, Hausen H. Parental influence on children's oral health-related behavior. Acta Odontol Scand. 2006;64(5):286-292.

14. Okada M, Kawamura M, Kaihara Y, et al. Influence of parents' oral health behaviour on oral health status of their school children: an exploratory study 
employing a causal modelling technique. Int J Paediatr Dent. 2002;12(2):101108.

15. Mohebbi SZ, Virtanen JI, Murtomaa H, Vahid-Golpayegani M, Vehkalahti MM. Mothers as facilitators of oral hygiene in early childhood. Int J Paediatr Dent. 2008;18(1):48-55.

16. Damle SG, Patil A, Jain S, Damle D, Chopal N. Effectiveness of supervised toothbrushing and oral health education in improving oral hygiene status and practices of urban and rural school children: A comparative study. J Int Soc Prev Community Dent. 2014;4(3):175-181.

17. Dye AB, Vargas CM, Lee JJ, Magder L, Tinanoff N. Assessing the Relationship Between Children's Oral Health Status and That of Their Mothers. J Am Dent Assoc. 2011;142 (2):173-183.

18. Vanagas G, Milasauskiene Z, Grabauskas V, Mickeviciene A. Associations between parental skills and their attitudes toward importance to develop good oral hygiene skills in their children. Medicina (Kaunas). 2009;45(9):718-723.

19. Pilebro C, Backman B. Teaching oral hygiene to children with autism. Int J Paediatr Dent. 2005;15(1):1-9.

20. Saied-Moallemi Z, Virtanen JI, Ghofranipour F, Murtomaa H. Influence of mothers' oral health knowledge and attitudes on their children's dental health. Eur Arch Paediatr Dent. 2008;9(2):79-83.

21. Byck GR, Walton SM, Cooksey JA. Access to dental care services for Medicaid children: variations by urban/rural categories in Illinois. J Rural Health. 2002;18.

22. Nash DA, Mathu-Muju KR, Freeman JW. Ensuring Access to Oral Health Care for Children: School-Based Care by Dental Therapists - A Commentary. J Sch Health. 2015;85(10).

23. Kinirons M, McCabe M. Familial and Maternal Factors Affecting the Dental Health and Dental Attendance of Preschool Children. Community Dent Health. 1995;12:226-229.

24. Crawford AN, Lennon MA. Dental attendance patterns among mothers and their children in an area of social deprivation. Community Dent Health. 1992;9:289294.

25. Amin MS. Utilization of dental services by children in low-income families in Alberta. J Can Dent Assoc 2011;77(b57).

26. Mitrakul K, Laovoravit V, Vanichanuwat V, Charatchaiwanna A, Bunpradit W, Arunakul M. Factors Associated with Parent Capability on Child's Oral Health Care. Southeast Asian J Trop Med Public Health. 2012;43(1):249-255.

27. Alsheri A, Nasim VS. Infant oral health care knowledge and awareness among parents in Abha city of Aseer Region, Saudi Arabia. Saudi J Dent Res. 2015.

28. Adeniyi AA, Ogunbodede EO, Jeboda SO, Morenike FO. Do maternal factors influence the dental health status of Nigerian pre-school children? Int J Paediatr Dent. 2009;19:448-454.

29. Skeie MS, Klock KS, Haugejorden O, Riordan PJ, Espelid I. Tracking of parents' attitudes to their children's oral health-related behavior-Oslo, Norway, 2002-04. Acta Odontol Scand. 2010;68(1):49-56.

30. Poutanen R, Lahti S, Seppä L, Tolvanen M, Hausen H. Oral health-related knowledge, attitudes, behavior, and family characteristics among Finnish 
schoolchildren with and without active initial caries lesions. Acta Odontol Scand. 2007;65(2):87-96.

31. Goettems ML, Ardenghi TM, Demarco FF, Romano AR, Torriani DD. Children's use of dental services: influence of maternal dental anxiety, attendance pattern, and perception of children's quality of life. Community Dent Oral Epidemiol. 2012;40:451-458.

32. Finlayson TL, Siefert K, Ismail AI, Sohn W. Maternal self-efficacy and 1-5-yearold children's brushing habits. Community Dent and Oral Epidemiol. 2007;35(4):272-281.

33. Pahel BT, Rozier RG, Slade GD. Parental perceptions of children's oral health: The Early Childhood Oral Health Impact Scale (ECOHIS). Health Qual Life Outcomes. 2007;5(6).

34. Suresh BS, Ravishankar TL, Chaitra TR, Mohapatra AK, Gupta V. Mother's knowledge about pre-school child's oral health. J Indian Soc Pedod Prev Dent. 2010;28(4):282.

35. Vargas CM, Ronzio CR, Hayes KL. Oral health status of children and adolescents by rural residence, United States. J Rural Health. 2003;19.

36. Mitsea AG, Karidis AG, Donta-Bakoyianni C, Spyropoulos ND. Oral health status in Greek children and teenagers, with disabilities. J Clin Pediatr Dent. 2002;26(1):111-118.

37. Barbosa TS, Gaviao MBD. Oral health-related quality of life in children: Part III. Is there agreement between parents in rating their children's oral health-related quality of life? A systematic review. Int J Dent Hyg. 2008;6:108-113.

38. Jokovic A, Locker D, Guyat G. How well do parents know their children? Implications for proxy reporting of child health-related quality of life. Q Life Res. 2004;13:1297-1307.

39. Dela Cruz GG, Rozier GR, Slade G. Dental Screening and Referral of Young Children by Pediatric Primary Care Providers. Pediatrics. 2004;114(5).

40. Kressin NR, Nunn ME, Singh H, et al. Pediatric clinicians can help reduce rates of early childhood caries: effects of a practice based intervention. Med Care. 2009;47(11):1121-1128.

41. Rozier RG, Slade GD, Zeldin LP, Wang H. Parents' satisfaction with preventive dental care for young children provided by nondental primary care providers. Pediatr Dent. 2005;27(4):313-322.

42. Institute for Digital Research and Education (IDRE). How do I interpret odds ratios in logistic regression? 2016;

http://www.ats.ucla.edu/stat/mult_pkg/faq/general/odds_ratio.htm. Accessed 3/24/16.

43. Efe E, Sarvan S. Self-reported Knowledge and Behaviors Related to Oral and dental Health in Turkish Children. Issues Compr Pediatr Nrs. 2007;30:133-146.

44. Cantrell C. The Role of Physicians in Children's Oral Health. State Health Policy Monitor. 2008;2(5):1-5.

45. Centers for Disease Control and Prevention. Oral and Dental health. 2014; http://www.cdc.gov/nchs/fastats/dental.htm. Accessed 3/24/2016. 
46. Filstrup SL, Briskie D, Da Fonseca M, Lawrence L, Wandera A, Inglehart MR. Early childhood caries and quality of life: child and parent perspectives. Pediatr Dent. 2003;25(5):431-440.

47. Department of Health Education and Human Services. Dental Disease is a Chronic Problem Among Low-Income Populations. Washington, D.C.: United States General Accounting Office; 2000.

48. Hamdan AM. The relationship between patient, parent and clinician perceived need and normative orthodontic treatment need. Eur J of Orthod. 2004;26(3):265271.

49. Wang H, Norton EC, Rozier RG. Effects of the State Children's Health Insurance Program on access to dental care and use of dental services. Health Serv Res. 2007;42(4):1544-1563.

50. Health Resources and Services Administration. Oral Health. 2011; http://www.hrsa.gov/publichealth/clinical/oralhealth/. Accessed 3/24/2016.

51. Bloom B, Cohen RA. Dental insurance for persons under age 65 years with private health insurance: United States, 2008. NCHS data brief 2010; http://www.cdc.gov/nchs/data/databriefs/db40.htm. Accessed 3/24/16. 


\section{APPENDIX A}

\section{Trend Analysis for Parental Assessment Based on No Instructions Received}

\begin{tabular}{|c|c|c|c|c|}
\hline \multirow{2}{*}{ Year } & \multicolumn{3}{|c|}{ Parental Assessment } & \multirow{2}{*}{ Total, N (\%) } \\
\cline { 2 - 4 } & Good, N (\%) & Fair, N (\%) & Poor, N (\%) & \\
\hline 2003 & $441(72.41)$ & $141(23.15)$ & $27(4.43)$ & $609(10.34)$ \\
\hline 2004 & $499(74.59)$ & $132(19.73)$ & $38(5.68)$ & $669(11.36)$ \\
\hline 2005 & $573(75.39)$ & $139(18.29)$ & $48(6.32)$ & $760(12.91)$ \\
\hline 2006 & $499(76.53)$ & $116(17.79)$ & $37(5.67)$ & $652(11.07)$ \\
\hline 2007 & $394(58.28)$ & $248(36.69)$ & $34(5.03)$ & $676(11.48)$ \\
\hline 2008 & $409(60.59)$ & $232(34.37)$ & $34(5.04)$ & $675(11.46)$ \\
\hline 2009 & $403(60.15)$ & $237(35.37)$ & $30(4.48)$ & $670(11.38)$ \\
\hline 2010 & $230(3.91)$ & $157(38.77)$ & $18(4.44)$ & $405(6.88)$ \\
\hline 2011 & $250(57.47)$ & $159(36.55)$ & $26(5.98)$ & $435(7.39)$ \\
\hline 2012 & & & & \\
\hline 2013 & $120(59.11)$ & $70(34.48)$ & $13(6.40)$ & $203(3.45)$ \\
\hline 2014 & $80(59.26)$ & $52(38.52)$ & $3(2.22)$ & $135(2.29)$ \\
\hline Total & $3,898(66.19)$ & $1,683(28.58)$ & $308(5.23)$ & $5,889(100.00)$ \\
\hline
\end{tabular}

\section{Trend Analysis for Parental Assessment Based on Instructions Received}

\begin{tabular}{|c|c|c|c|c|}
\hline \multirow{2}{*}{ Year } & \multicolumn{3}{|c|}{ Parental Assessment } & \multirow{2}{*}{ Total, N (\%) } \\
\cline { 2 - 4 } & Good, N (\%) & Fair, N (\%) & Poor, N (\%) & \\
\hline 2003 & $2,486(83.62)$ & $423(14.23)$ & $64(2.15)$ & $2,973(10.79)$ \\
\hline 2004 & $2,169(82.82)$ & $371(14.17)$ & $79(3.02)$ & $2,619(9.51)$ \\
\hline 2005 & $2,418(82.36)$ & $417(14.20)$ & $101(3.44)$ & $2,936(10.66)$ \\
\hline 2006 & $2,357(83.94)$ & $363(12.93)$ & $88(3.13)$ & $2,808(10.19)$ \\
\hline 2007 & $1,694(65.89)$ & $791(30.77)$ & $86(3.35)$ & $2,571(9.33)$ \\
\hline 2008 & $1,642(65.16)$ & $791(31.39)$ & $87(3.45)$ & $2,520(9.15)$ \\
\hline 2009 & $1,924(67.75)$ & $832(29.30)$ & $84(2.96)$ & $2,840(10.31)$ \\
\hline 2010 & $1,729(69.35)$ & $717(28.76)$ & $47(1.89)$ & $2,493(9.05)$ \\
\hline 2011 & $1,921(70.89)$ & $742(27.38)$ & $47(1.73)$ & $2,710(9.84)$ \\
\hline 2012 & & & & \\
\hline 2013 & $1,220(69.79)$ & $490(28.03)$ & $38(2.17)$ & $1,748(6.35)$ \\
\hline 2014 & $999(75.40)$ & $306(23.09)$ & $20(1.51)$ & $1,325(4.81)$ \\
\hline Total & $20,559(74.64)$ & $6,243(22.67)$ & $741(2.69)$ & $27,543(100.00)$ \\
\hline
\end{tabular}


Trend Analysis for Clinical Screening with No Instructions Received

\begin{tabular}{|c|c|c|c|c|}
\hline \multirow{2}{*}{ Year } & \multicolumn{3}{|c|}{ Clinical Screening } & \multirow{2}{*}{ Total, N (\%) } \\
\cline { 2 - 4 } & Good, N (\%) & Fair, N (\%) & Poor, N (\%) & \\
\hline 2003 & $407(66.83)$ & $163(26.77)$ & $39(6.40)$ & $609(10.34)$ \\
\hline 2004 & $466(69.66)$ & $159(23.77)$ & $44(6.58)$ & $669(11.36)$ \\
\hline 2005 & $493(64.87)$ & $214(28.16)$ & $53(6.97)$ & $760(12.91)$ \\
\hline 2006 & $387(59.36)$ & $206(31.60)$ & $59(9.05)$ & $652(11.07)$ \\
\hline 2007 & $460(68.05)$ & $159(23.52)$ & $57(8.43)$ & $676(11.48)$ \\
\hline 2008 & $423(62.67)$ & $174(25.78)$ & $78(11.56)$ & $675(11.46)$ \\
\hline 2009 & $475(70.90)$ & $141(21.04)$ & $54(8.06)$ & $670(11.38)$ \\
\hline 2010 & $276(68.15)$ & $82(20.25)$ & $47(11.60)$ & $405(6.88)$ \\
\hline 2011 & $303(69.66)$ & $95(21.84)$ & $37(8.51)$ & $435(7.39)$ \\
\hline 2012 & & & & $203(3.45)$ \\
\hline 2013 & $139(68.47)$ & $54(26.60)$ & $10(4.93)$ & $135(2.29)$ \\
\hline 2014 & $93(68.89)$ & $34(25.19)$ & $8(5.93)$ & $5,889(100.00)$ \\
\hline Total & $3,922(66.60)$ & $1,481(25.15)$ & $486(8.25)$ & \\
\hline
\end{tabular}

Trend Analysis for Clinical Screening Based on Instructions Received

\begin{tabular}{|c|c|c|c|c|}
\hline \multirow{2}{*}{ Year } & \multicolumn{3}{|c|}{ Clinical Screening } & \multirow{2}{*}{ Total, N (\%) } \\
\cline { 2 - 4 } & Good, N (\%) & Fair, N (\%) & Poor, N (\%) & \\
\hline 2003 & $2,247(75.58)$ & $612(20.59)$ & $114(3.83)$ & $2,973(10.79)$ \\
\hline 2004 & $1,908(72.85)$ & $606(23.14)$ & $105(4.01)$ & $2,619(9.51)$ \\
\hline 2005 & $2,091(71.22)$ & $721(24.56)$ & $124(4.22)$ & $2,936(10.66)$ \\
\hline 2006 & $1,926(68.59)$ & $705(25.11)$ & $177(6.30)$ & $2,808(10.19)$ \\
\hline 2007 & $1,903(74.02)$ & $498(19.37)$ & $170(6.61)$ & $2,571(9.33)$ \\
\hline 2008 & $1,774(70.40)$ & $510(20.24)$ & $236(9.37)$ & $2,520(9.15)$ \\
\hline 2009 & $2,205(77.64)$ & $474(16.69)$ & $161(5.67)$ & $2,840(10.31)$ \\
\hline 2010 & $1,891(75.85)$ & $462(18.53)$ & $140(5.62)$ & $2,493(9.05)$ \\
\hline 2011 & $2,044(75.42)$ & $476(17.56)$ & $190(7.01)$ & $2,710(9.84)$ \\
\hline 2012 & & & & \\
\hline 2013 & $1,328(75.97)$ & $342(19.57)$ & $78(4.46)$ & $1,748(6.35)$ \\
\hline 2014 & $1029(77.66)$ & $246(18.57)$ & $50(3.77)$ & $1,325(4.81)$ \\
\hline Total & $20,346(73.87)$ & $5,652(20.52)$ & $1,545(5.61)$ & 27,543 \\
& & & & $(100.00)$ \\
\hline
\end{tabular}




\section{Trend Analysis for 'GOOD' Parental Assessment Vs Clinical Screening}

\begin{tabular}{|c|c|c|c|c|}
\hline \multirow{2}{*}{ Year } & \multicolumn{3}{|c|}{ Parental Assessment-GOOD } & \multirow{2}{*}{ Total, N (\%) } \\
\cline { 2 - 4 } & Good, N(\%) & Fair, N (\%) & Poor, N (\%) & \\
\hline 2003 & $2,291(78.27)$ & $563(19.23)$ & $73(2.49)$ & $2,927(11.97)$ \\
\hline 2004 & $2,043(76.57)$ & $554(20.76)$ & $71(2.66)$ & $2,668(10.91)$ \\
\hline 2005 & $2,214(74.02)$ & $691(23.10)$ & $86(2.88)$ & $2,991(12.23)$ \\
\hline 2006 & $2,029(71.04)$ & $703(24.61)$ & $124(4.34)$ & $2,856(11.68)$ \\
\hline 2007 & $1,637(78.40)$ & $361(17.29)$ & $90(4.31)$ & $2,088(8.54)$ \\
\hline 2008 & $1,581(77.08)$ & $356(17.36)$ & $114(5.56)$ & $2,051(8.39)$ \\
\hline 2009 & $1,953(83.93)$ & $309(13.28)$ & $65(2.79)$ & $2,327(9.51)$ \\
\hline 2010 & $1,587(81.01)$ & $305(15.57)$ & $67(3.42)$ & $1,959(8.01)$ \\
\hline 2011 & $1,746(80.42)$ & $329(15.15)$ & $96(4.42)$ & $2,171(8.88)$ \\
\hline 2012 & & & & \\
\hline 2013 & $1,091(81.42)$ & $221(16.49)$ & $28(2.09)$ & $1,340(5.48)$ \\
\hline 2014 & $879(81.46)$ & $179(16.59)$ & $21(1.95)$ & $1,079(4.41)$ \\
\hline Total & $19,051(77.90)$ & $4,571(18.69)$ & $835(3.41)$ & 24,457 \\
& & & & $(100.00)$ \\
\hline
\end{tabular}

Trend Analysis for 'FAIR' Parental Assessment Vs Clinical Screening

\begin{tabular}{|c|c|c|c|c|}
\hline \multirow{2}{*}{ Year } & \multicolumn{3}{|c|}{ Parental Assessment- FAIR } & \multirow{2}{*}{ Total, N (\%) } \\
\cline { 2 - 4 } & Good, N (\%) & Fair, N (\%) & Poor, N (\%) & \\
\hline 2003 & $314(55.67)$ & $190(33.69)$ & $60(10.64)$ & $564(7.12)$ \\
\hline 2004 & $269(53.48)$ & $178(35.39)$ & $56(11.13)$ & $503(6.35)$ \\
\hline 2005 & $292(52.52)$ & $202(36.33)$ & $62(11.15)$ & $556(7.01)$ \\
\hline 2006 & $227(47.39)$ & $167(34.86)$ & $85(17.75)$ & $479(6.04)$ \\
\hline 2007 & $670(64.49)$ & $261(25.12)$ & $108(10.39)$ & $1,039(13.11)$ \\
\hline 2008 & $577(56.40)$ & $293(28.64)$ & $153(14.96)$ & $1,023(12.91)$ \\
\hline 2009 & $681(63.70)$ & $278(26.01)$ & $110(10.29)$ & $1,069(13.49)$ \\
\hline 2010 & $560(64.07)$ & $215(24.60)$ & $99(11.33)$ & $874(11.03)$ \\
\hline 2011 & $568(63.04)$ & $221(24.53)$ & $112(12.43)$ & $901(11.37)$ \\
\hline 2012 & & & & \\
\hline 2013 & $352(62.86)$ & $159(28.39)$ & $49(8.75)$ & $560(7.07)$ \\
\hline 2014 & $230(64.25)$ & $96(26.82)$ & $32(8.94)$ & $358(4.52)$ \\
\hline Total & $4,740(59.80)$ & $2,260(28.51)$ & $926(11.68)$ & $7,926(100.00)$ \\
\hline
\end{tabular}


Trend Analysis for 'POOR' Parental Assessment Vs Clinical Screening

\begin{tabular}{|c|c|c|c|c|}
\hline \multirow{2}{*}{ Year } & \multicolumn{2}{|c|}{ Parental Assessment-POOR } & \multirow{2}{*}{ Total, N (\%) } \\
\cline { 2 - 4 } & Good, N (\%) & Fair, N (\%) & Poor, N (\%) & \\
\hline 2003 & $49(53.85)$ & $22(24.18)$ & $20(21.98)$ & $91(8.67)$ \\
\hline 2004 & $62(52.99)$ & $33(28.21)$ & $22(18.80)$ & $117(11.15)$ \\
\hline 2005 & $78(52.35)$ & $42(28.19)$ & $29(19.46)$ & $149(14.20)$ \\
\hline 2006 & $57(45.60)$ & $41(32.80)$ & $27(21.60)$ & $125(11.92)$ \\
\hline 2007 & $56(46.67)$ & $35(29.17)$ & $29(24.17)$ & $120(11.44)$ \\
\hline 2008 & $39(32.23)$ & $35(28.93)$ & $47(38.84)$ & $121(11.53)$ \\
\hline 2009 & $46(40.35)$ & $28(24.56)$ & $40(35.09)$ & $114(10.87)$ \\
\hline 2010 & $20(30.77)$ & $24(36.92)$ & $21(32.31)$ & $65(6.20)$ \\
\hline 2011 & $33(45.21)$ & $21(28.77)$ & $19(26.03)$ & $73(6.96)$ \\
\hline 2012 & & & & \\
\hline 2013 & $24(47.06)$ & $16(31.37)$ & $11(21.57)$ & $51(4.86)$ \\
\hline 2014 & $13(56.52)$ & $05(21.74)$ & $05(21.74)$ & $23(2.19)$ \\
\hline Total & $477(45.47)$ & $302(28.79)$ & $270(25.74)$ & $1,049(100.00)$ \\
\hline
\end{tabular}

\section{Trend Analysis for Parental Assessment with No Instructions Received from Dental} office

\begin{tabular}{|c|c|c|c|c|}
\hline \multirow{2}{*}{ Year } & \multicolumn{2}{|c|}{ Parental Assessment-Dental office } & \multirow{2}{*}{ Total, N (\%) } \\
\cline { 2 - 4 } & Good, N (\%) & Fair, N (\%) & Poor, N (\%) & \\
\hline 2003 & $441(72.4)$ & $141(23.2)$ & $27(4.4)$ & $60(10.3)$ \\
\hline 2004 & $499(74.6)$ & $132(19.7)$ & $38(5.7)$ & $669(11.4)$ \\
\hline 2005 & $573(75.4)$ & $139(18.3)$ & $48(6.3)$ & $760(12.9)$ \\
\hline 2006 & $499(76.5)$ & $116(17.8)$ & $37(5.7)$ & $652(11.1)$ \\
\hline 2007 & $394(58.3)$ & $248(36.7)$ & $34(5.0)$ & $676(11.5)$ \\
\hline 2008 & $409(60.6)$ & $232(34.4)$ & $34(5.0)$ & $675(11.5)$ \\
\hline 2009 & $403(60.1)$ & $237(35.4)$ & $30(4.5)$ & $670(11.4)$ \\
\hline 2010 & $230(56.8)$ & $157(38.8)$ & $18(4.4)$ & $405(6.9)$ \\
\hline 2011 & $250(57.5)$ & $159(36.6)$ & $26(6.0)$ & $435(7.4)$ \\
\hline 2012 & & & & \\
\hline 2013 & $120(59.1)$ & $70(34.5)$ & $13(6.4)$ & $203(3.4)$ \\
\hline 2014 & $80(59.3)$ & $52(38.5)$ & $3(2.2)$ & $135(2.3)$ \\
\hline Total & $3,898(66.2)$ & $1,683(28.6)$ & $308(5.2)$ & $5,889(100.0)$ \\
\hline
\end{tabular}


Trend Analysis for Parental Assessment Based on Instructions Received from $\underline{\text { Dental office }}$

\begin{tabular}{|c|c|c|c|c|}
\hline \multirow{2}{*}{ Year } & \multicolumn{2}{|c|}{ Parental Assessment-Dental office } & \multirow{2}{*}{ Total, N (\%) } \\
\cline { 2 - 4 } & Good, N (\%) & Fair, N (\%) & Poor, N (\%) & \\
\hline 2003 & $2,112(84.3)$ & $345(13.8)$ & $48(1.9)$ & $2,505(11.2)$ \\
\hline 2004 & $1,715(83.0)$ & $292(14.1)$ & $60(2.9)$ & $2,067(9.3)$ \\
\hline 2005 & $1,946(82.9)$ & $336(14.3)$ & $66(2.8)$ & $2,348(10.5)$ \\
\hline 2006 & $1,875(84.2)$ & $286(12.8)$ & $66(3.0)$ & $2,227(10.0)$ \\
\hline 2007 & $1,347(67.0)$ & $602(30.0)$ & $60(3.0)$ & $2,009(9.0)$ \\
\hline 2008 & $1,323(66.7)$ & $603(30.4)$ & $58(2.9)$ & $1,984(8.9)$ \\
\hline 2009 & $1,598(68.8)$ & $671(28.9)$ & $53(2.3)$ & $2,322(10.4)$ \\
\hline 2010 & $1,422(69.7)$ & $588(28.8)$ & $31(1.5)$ & $2,041(9.2)$ \\
\hline 2011 & $1,581(71.6)$ & $594(26.9)$ & $32(1.4)$ & $2,207(9.9)$ \\
\hline 2012 & & & & \\
\hline 2013 & $1,035(70.6)$ & $406(27.7)$ & $26(1.8)$ & $1,467(6.6)$ \\
\hline 2014 & $857(77.3)$ & $238(21.5)$ & $13(1.2)$ & $1,108(5.0)$ \\
\hline Total & $16,811(75.4)$ & $4,961(22.3)$ & $513(2.3)$ & $22,285(100.0)$ \\
\hline
\end{tabular}

Trend Analysis for Parental Assessment with and without Instructions from Dental office

\begin{tabular}{|c|c|c|c|c|}
\hline \multirow{2}{*}{ Year } & \multicolumn{2}{|c|}{ Parental Assessment-Dental office } & \multirow{2}{*}{ Total, N (\%) } \\
\cline { 2 - 4 } & Good, N(\%) & Fair, N (\%) & Poor, N (\%) & \\
\hline 2003 & $2,553(82.0)$ & $486(15.6)$ & $75(2.4)$ & $3,114(11.1)$ \\
\hline 2004 & $2,214(80.9)$ & $424(15.5)$ & $98(3.6)$ & $2,736(9.7)$ \\
\hline 2005 & $2,519(81.0)$ & $475(15.3)$ & $114(3.7)$ & $3,108(11.0)$ \\
\hline 2006 & $2,374(82.5)$ & $402(14.0)$ & $103(3.6)$ & $2,879(10.2)$ \\
\hline 2007 & $1,741(64.8)$ & $850(31.7)$ & $94(3.5)$ & $2,685(9.5)$ \\
\hline 2008 & $1,732(65.1)$ & $835(31.4)$ & $92(3.5)$ & $2,659(9.4)$ \\
\hline 2009 & $2,001(66.9)$ & $908(30.3)$ & $83(2.8)$ & $2,992(10.6)$ \\
\hline 2010 & $1,652(67.5)$ & $745(30.5)$ & $49(2.0)$ & $2,446(8.7)$ \\
\hline 2011 & $1,831(69.3)$ & $753(28.5)$ & $58(2.2)$ & $2,642(9.4)$ \\
\hline 2012 & & & & \\
\hline 2013 & $1,155(69.2)$ & $476(28.5)$ & $39(2.3)$ & $1,670(5.9)$ \\
\hline 2014 & $937(75.4)$ & $290(23.3)$ & $16(1.3)$ & $1,243(4.4)$ \\
\hline Total & $20,709(73.5)$ & $6,644(23.6)$ & $821(2.9)$ & $28,174(100.0)$ \\
\hline
\end{tabular}


Trend Analysis for Parental Assessment with Instructions Received from Physician $\underline{\text { Office }}$

\begin{tabular}{|c|c|c|c|c|}
\hline \multirow{2}{*}{ Year } & \multicolumn{2}{|c|}{ Parental Assessment-Physician Office } & \multirow{2}{*}{ Total, N (\%) } \\
\cline { 2 - 4 } & Good, N (\%) & Fair, N (\%) & Poor, N (\%) & \\
\hline 2003 & $31(75.6)$ & $8(19.5)$ & $2(4.9)$ & $41(7.0)$ \\
\hline 2004 & $57(79.2)$ & $14(19.4)$ & $1(1.4)$ & $72(12.3)$ \\
\hline 2005 & $67(77.9)$ & $11(12.8)$ & $8(9.3)$ & $86(14.7)$ \\
\hline 2006 & $61(81.3)$ & $13(17.3)$ & $1(1.3)$ & $75(12.8)$ \\
\hline 2007 & $18(43.9)$ & $23(56.1)$ & $0(0)$ & $41(7.0)$ \\
\hline 2008 & $25(49.0)$ & $23(45.1)$ & $3(5.9)$ & $51(8.7)$ \\
\hline 2009 & $26(56.5)$ & $18(39.1)$ & $2(4.3)$ & $46(7.9)$ \\
\hline 2010 & $19(44.2)$ & $21(48.8)$ & $3(7.0)$ & $43(7.4)$ \\
\hline 2011 & $37(69.8)$ & $13(24.5)$ & $3(5.7)$ & $53(9.1)$ \\
\hline 2012 & & & & \\
\hline 2013 & $16(64.0)$ & $8(32.0)$ & $1(4.0)$ & $25(4.3)$ \\
\hline 2014 & $31(60.8)$ & $19(37.3)$ & $1(2.0)$ & $51(8.7)$ \\
\hline Total & $388(66.4)$ & $171(29.3)$ & $25(4.3)$ & $584(100.0)$ \\
\hline
\end{tabular}

Trend Analysis for Parental Assessment with and without Instructions from Physician Office

\begin{tabular}{|c|c|c|c|c|}
\hline \multirow{2}{*}{ Year } & \multicolumn{3}{|c|}{ Parental Assessment-Physician Office } & \multirow{2}{*}{ Total, N (\%) } \\
\cline { 2 - 4 } & Good, N(\%) & Fair, N (\%) & Poor, N (\%) & \\
\hline 2003 & $472(72.6)$ & $149(22.9)$ & $29(4.5)$ & $650(10.0)$ \\
\hline 2004 & $556(75.0)$ & $146(19.7)$ & $39(5.3)$ & $741(11.4)$ \\
\hline 2005 & $640(75.7)$ & $150(17.7)$ & $56(6.6)$ & $846(13.1)$ \\
\hline 2006 & $560(77.0)$ & $129(17.7)$ & $38(5.2)$ & $727(11.2)$ \\
\hline 2007 & $412(57.5)$ & $271(37.8)$ & $34(4.7)$ & $717(11.1)$ \\
\hline 2008 & $434(59.8)$ & $255(35.1)$ & $37(5.1)$ & $726(11.2)$ \\
\hline 2009 & $429(59.9)$ & $255(35.6)$ & $32(4.5)$ & $716(11.1)$ \\
\hline 2010 & $249(55.6)$ & $178(39.7)$ & $21(4.7)$ & $448(6.9)$ \\
\hline 2011 & $287(6.7)$ & $172(35.2)$ & $29(5.9)$ & $488(7.5)$ \\
\hline 2012 & & & & \\
\hline 2013 & $136(59.6)$ & $78(34.2)$ & $14(6.1)$ & $228(3.5)$ \\
\hline 2014 & $111(59.7)$ & $71(38.2)$ & $4(2.2)$ & $186(2.9)$ \\
\hline Total & $4,286(66.2)$ & $1,854(28.6)$ & $333(5.1)$ & $6,473(100.0)$ \\
\hline
\end{tabular}


Trend Analysis for Parental Assessment with Instructions Received from Health Department

\begin{tabular}{|c|c|c|c|c|}
\hline \multirow{2}{*}{ Year } & \multicolumn{3}{|c|}{ Parental Assessment-Health Department } & \multirow{2}{*}{ Total, N (\%) } \\
\cline { 2 - 4 } & Good, N(\%) & Fair, N (\%) & Poor, N (\%) & \\
\hline 2003 & $19(55.9)$ & $11(32.4)$ & $4(11.8)$ & $34(9.6)$ \\
\hline 2004 & $26(78.8)$ & $4(12.1)$ & $3(9.1)$ & $33(9.3)$ \\
\hline 2005 & $35(76.1)$ & $6(13.0)$ & $5(10.9)$ & $46(13.0)$ \\
\hline 2006 & $30(76.9)$ & $7(17.9)$ & $2(5.1)$ & $39(11.0)$ \\
\hline 2007 & $16(50.0)$ & $15(46.9)$ & $1(3.1)$ & $32(9.0)$ \\
\hline 2008 & $16(34.8)$ & $27(58.7)$ & $3(6.5)$ & $46(13.0)$ \\
\hline 2009 & $10(34.5)$ & $12(41.4)$ & $7(24.1)$ & $29(8.2)$ \\
\hline 2010 & $13(52.0)$ & $9(36.0)$ & $2(8.0)$ & $25(7.0)$ \\
\hline 2011 & $14(56.0)$ & $9(36.0)$ & $2(8.0)$ & $25(7.0)$ \\
\hline 2012 & & & & \\
\hline 2013 & $10(55.6)$ & $6(33.3)$ & $2(11.1)$ & $18(5.1)$ \\
\hline 2014 & $16(57.1)$ & $11(39.3)$ & $1(3.6)$ & $28(7.9)$ \\
\hline Total & $205(57.7)$ & $117(33.0)$ & $33(9.3)$ & $355(100.0)$ \\
\hline
\end{tabular}

Trend Analysis for Parental Assessment with and without Instructions from Health Department

\begin{tabular}{|c|c|c|c|c|}
\hline \multirow{2}{*}{ Year } & \multicolumn{3}{|c|}{ Parental Assessment-Health Department } & \multirow{2}{*}{ Total, N (\%) } \\
\cline { 2 - 4 } & Good, N (\%) & Fair, N (\%) & Poor, N(\%) & \\
\hline 2003 & $460(71.5)$ & $152(23.6)$ & $31(4.8)$ & $643(10.3)$ \\
\hline 2004 & $525(74.8)$ & $136(19.4)$ & $41(5.8)$ & $702(11.2)$ \\
\hline 2005 & $608(75.4)$ & $145(18.0)$ & $53(6.6)$ & $806(12.9)$ \\
\hline 2006 & $529(76.6)$ & $123(17.8)$ & $39(5.6)$ & $691(11.1)$ \\
\hline 2007 & $410(57.9)$ & $263(37.1)$ & $35(4.9)$ & $708(11.3)$ \\
\hline 2008 & $425(58.9)$ & $259(35.9)$ & $37(5.1)$ & $721(11.5)$ \\
\hline 2009 & $413(59.1)$ & $249(35.6)$ & $37(5.3)$ & $699(11.2)$ \\
\hline 2010 & $243(56.5)$ & $166(38.6)$ & $21(4.9)$ & $430(6.9)$ \\
\hline 2011 & $264(57.4)$ & $168(36.5)$ & $28(6.1)$ & $460(7.4)$ \\
\hline 2012 & & & & \\
\hline 2013 & $130(58.8)$ & $76(34.4)$ & $15(6.8)$ & $221(3.5)$ \\
\hline 2014 & $96(58.9)$ & $63(38.7)$ & $4(2.5)$ & $163(2.6)$ \\
\hline Total & $4,103(65.7)$ & $1,800(28.8)$ & $341(5.5)$ & $6,244(100.0)$ \\
\hline
\end{tabular}


Trend Analysis for Parental Assessment with Instructions Received from Other Providers

\begin{tabular}{|c|c|c|c|c|}
\hline \multirow{2}{*}{ Year } & \multicolumn{3}{|c|}{ Parental Assessment-Other Providers } & \multirow{2}{*}{ Total, N (\%) } \\
\cline { 2 - 4 } & Good, N(\%) & Fair, N (\%) & Poor, N (\%) & \\
\hline 2003 & $61(70.9)$ & $22(25.6)$ & $3(3.5)$ & $86(10.6)$ \\
\hline 2004 & $79(76.0)$ & $22(21.2)$ & $3(2.9)$ & $104(12.8)$ \\
\hline 2005 & $71(67.6)$ & $21(20.0)$ & $13(12.4)$ & $105(12.9)$ \\
\hline 2006 & $65(77.4)$ & $10(11.9)$ & $9(10.7)$ & $84(10.3)$ \\
\hline 2007 & $39(55.7)$ & $26(37.1)$ & $5(7.1)$ & $70(8.6)$ \\
\hline 2008 & $32(41.6)$ & $36(46.8)$ & $9(11.7)$ & $77(9.4)$ \\
\hline 2009 & $31(45.6)$ & $28(41.2)$ & $9(13.2)$ & $68(8.3)$ \\
\hline 2010 & $32(50.0)$ & $29(45.3)$ & $3(4.7)$ & $64(7.9)$ \\
\hline 2011 & $31(44.9)$ & $36(52.2)$ & $2(2.9)$ & $69(8.5)$ \\
\hline 2012 & & & & \\
\hline 2013 & $21(52.5)$ & $17(42.5)$ & $2(5.0)$ & $40(4.9)$ \\
\hline 2014 & $29(60.4)$ & $17(35.4)$ & $2(4.2)$ & $48(5.9)$ \\
\hline Total & $491(60.2)$ & $264(32.4)$ & $60(7.4)$ & $815(100.0)$ \\
\hline
\end{tabular}

Trend Analysis for Parental Assessment with and without Instructions from Other Providers

\begin{tabular}{|c|c|c|c|c|}
\hline \multirow{2}{*}{ Year } & \multicolumn{3}{|c|}{ Parental Assessment-Other Providers } & \multirow{2}{*}{ Total, N (\%) } \\
\cline { 2 - 4 } & Good, N(\%) & Fair, N (\%) & Poor, N (\%) & \\
\hline 2003 & $502(72.2)$ & $163(23.5)$ & $30(4.3)$ & $695(10.4)$ \\
\hline 2004 & $578(74.8)$ & $154(19.9)$ & $41(5.3)$ & $773(11.5)$ \\
\hline 2005 & $644(74.5)$ & $160(18.5)$ & $61(7.1)$ & $865(12.9)$ \\
\hline 2006 & $564(76.6)$ & $126(17.1)$ & $46(6.2)$ & $736(11.0)$ \\
\hline 2007 & $433(58.0)$ & $274(36.7)$ & $39(5.2)$ & $746(11.1)$ \\
\hline 2008 & $441(58.6)$ & $268(35.6)$ & $43(5.7)$ & $752(11.2)$ \\
\hline 2009 & $434(58.8)$ & $265(35.9)$ & $39(5.3)$ & $738(11.0)$ \\
\hline 2010 & $262(55.9)$ & $186(39.7)$ & $21(4.5)$ & $469(7.0)$ \\
\hline 2011 & $281(55.8)$ & $195(38.7)$ & $28(5.6)$ & $504(7.5)$ \\
\hline 2012 & & & & \\
\hline 2013 & $141(58.0)$ & $87(35.8)$ & $15(5.2)$ & $243(3.6)$ \\
\hline 2014 & $109(59.6)$ & $69(37.7)$ & $5(2.7)$ & $183(2.7)$ \\
\hline Total & $4389(65.5)$ & $1947(29.0)$ & $368(5.5)$ & $6704(100.0)$ \\
\hline
\end{tabular}


Trend Analysis for Clinical Screening with No Instructions Received from Dental office

\begin{tabular}{|c|c|c|c|c|}
\hline \multirow{2}{*}{ Year } & \multicolumn{3}{|c|}{ Clinical Screening-Dental Office } & \multirow{2}{*}{ Total, N (\%) } \\
\cline { 2 - 4 } & Good, N (\%) & Fair, N (\%) & Poor, N (\%) & \\
\hline 2003 & $407(66.8)$ & $163(26.8)$ & $39(6.4)$ & $609(10.3)$ \\
\hline 2004 & $466(69.7)$ & $159(23.8)$ & $44(6.6)$ & $669(11.4)$ \\
\hline 2005 & $493(64.9)$ & $214(28.2)$ & $53(7.0)$ & $760(12.9)$ \\
\hline 2006 & $387(59.4)$ & $206(31.6)$ & $59(9.0)$ & $652(11.1)$ \\
\hline 2007 & $460(68.0)$ & $159(23.5)$ & $57(8.4)$ & $676(11.5)$ \\
\hline 2008 & $423(62.7)$ & $174(25.8)$ & $78(11.6)$ & $675(11.5)$ \\
\hline 2009 & $475(70.9)$ & $141(21.0)$ & $54(8.1)$ & $670(11.4)$ \\
\hline 2010 & $276(68.1)$ & $82(20.2)$ & $47(11.6)$ & $405(6.9)$ \\
\hline 2011 & $303(69.7)$ & $95(21.8)$ & $37(8.5)$ & $435(7.4)$ \\
\hline 2012 & & & & \\
\hline 2013 & $139(68.5)$ & $54(26.6)$ & $10(4.9)$ & $203(3.4)$ \\
\hline 2014 & $93(68.9)$ & $34(25.2)$ & $8(5.9)$ & $135(2.3)$ \\
\hline Total & $3,922(66.6)$ & $1,481(25.1)$ & $486(8.3)$ & $5,889(100.0)$ \\
\hline
\end{tabular}

\section{Trend Analysis for Clinical Screening Based on Instructions Received from Dental}

\section{Office}

\begin{tabular}{|c|c|c|c|c|}
\hline \multirow{2}{*}{ Year } & \multicolumn{3}{|c|}{ Parental Assessment-Dental Office } & \multirow{2}{*}{ Total, N (\%) } \\
\cline { 2 - 4 } & Good, N (\%) & Fair, N (\%) & Poor, N (\%) & \\
\hline 2003 & $1,914(76.4)$ & $507(20.2)$ & $84(3.4)$ & $2,505(11.2)$ \\
\hline 2004 & $1,500(72.6)$ & $484(23.4)$ & $83(4.0)$ & $2,067(9.3)$ \\
\hline 2005 & $1,698(72.3)$ & $567(24.1)$ & $83(3.5)$ & $2,348(10.5)$ \\
\hline 2006 & $1,546(69.4)$ & $558(25.1)$ & $123(5.5)$ & $2,227(10.0)$ \\
\hline 2007 & $1,502(74.8)$ & $377(18.8)$ & $130(6.5)$ & $2,009(9.0)$ \\
\hline 2008 & $1,420(71.6)$ & $398(20.1)$ & $166(8.4)$ & $1,984(8.9)$ \\
\hline 2009 & $1,826(78.6)$ & $371(16.0)$ & $125(5.4)$ & $2,322(10.4)$ \\
\hline 2010 & $1,554(76.1)$ & $374(18.3)$ & $113(5.5)$ & $2,041(9.2)$ \\
\hline 2011 & $1,691(10.2)$ & $364(16.5)$ & $152(6.9)$ & $2,207(9.9)$ \\
\hline 2012 & & & & \\
\hline 2013 & $1,122(76.5)$ & $280(19.1)$ & $65(4.4)$ & $1,467(6.6)$ \\
\hline 2014 & $873(78.8)$ & $200(18.1)$ & $35(3.2)$ & $1,108(5.0)$ \\
\hline Total & $16,646(74.7)$ & $4,480(20.1)$ & $1,159(5.2)$ & $22,285(100.0)$ \\
\hline
\end{tabular}


Trend Analysis for Clinical Screening with and Without Instructions from Dental $\underline{\text { Office }}$

\begin{tabular}{|c|c|c|c|c|}
\hline \multirow{2}{*}{ Year } & \multicolumn{3}{|c|}{ Clinical Screening-Dental Office } & \multirow{2}{*}{ Total, N (\%) } \\
\cline { 2 - 4 } & Good, N (\%) & Fair, N (\%) & Poor, N (\%) & \\
\hline 2003 & $2,321(74.5)$ & $670(21.5)$ & $123(3.9)$ & $3,114(11.1)$ \\
\hline 2004 & $1,966(71.9)$ & $643(23.5)$ & $127(4.6)$ & $2,736(9.7)$ \\
\hline 2005 & $2,191(70.5)$ & $781(25.1)$ & $136(4.4)$ & $3,108(11.0)$ \\
\hline 2006 & $1,933(67.1)$ & $764(26.5)$ & $182(6.3)$ & $2,879(10.2)$ \\
\hline 2007 & $1,962(73.1)$ & $536(20.0)$ & $187(7.0)$ & $2,685(9.5)$ \\
\hline 2008 & $1,843(69.3)$ & $572(21.5)$ & $244(9.2)$ & $2,659(9.4)$ \\
\hline 2009 & $2,301(76.9)$ & $512(17.1)$ & $179(6.0)$ & $2,992(10.6)$ \\
\hline 2010 & $1,830(74.8)$ & $456(7.6)$ & $160(6.5)$ & $2,446(8.7)$ \\
\hline 2011 & $1,994(75.5)$ & $459(17.4)$ & $189(7.2)$ & $2,643(9.4)$ \\
\hline 2012 & & & & \\
\hline 2013 & $1,261(75.5)$ & $334(20.0)$ & $75(4.5)$ & $1,670(5.9)$ \\
\hline 2014 & $966(77.7)$ & $234(18.8)$ & $43(3.5)$ & $1,243(4.4)$ \\
\hline Total & $20,568(73.0)$ & $5,961(21.2)$ & $1,645(5.8)$ & $28,174(100.0)$ \\
\hline
\end{tabular}

\section{Trend Analysis for Clinical Screening Based on Instructions Received from}

\section{Physician Office}

\begin{tabular}{|c|c|c|c|c|}
\hline \multirow{2}{*}{ Year } & \multicolumn{3}{|c|}{ Clinical Screening-Physician Office } & \multirow{2}{*}{ Total, N (\%) } \\
\cline { 2 - 4 } & Good, N(\%) & Fair, N (\%) & Poor, N (\%) & \\
\hline 2003 & $30(73.2)$ & $8(19.5)$ & $3(7.3)$ & $41(7.0)$ \\
\hline 2004 & $53(73.6)$ & $15(20.8)$ & $4(5.6)$ & $72(12.3)$ \\
\hline 2005 & $51(59.3)$ & $28(32.6)$ & $7(8.1)$ & $86(14.7)$ \\
\hline 2006 & $49(65.3)$ & $17(22.7)$ & $9(12.0)$ & $75(12.8)$ \\
\hline 2007 & $30(73.2)$ & $7(17.1)$ & $4(9.8)$ & $41(7.0)$ \\
\hline 2008 & $26(51.0)$ & $15(29.4)$ & $10(19.6)$ & $51(8.7)$ \\
\hline 2009 & $30(65.2)$ & $11(23.9)$ & $5(10.9)$ & $46(7.9)$ \\
\hline 2010 & $26(60.5)$ & $12(27.9)$ & $5(11.6)$ & $43(7.4)$ \\
\hline 2011 & $34(64.1)$ & $14(26.4)$ & $5(9.4)$ & $53(9.1)$ \\
\hline 2012 & & & & \\
\hline 2013 & $18(72.0)$ & $5(20.0)$ & $2(8.0)$ & $25(4.3)$ \\
\hline 2014 & $13(25.5)$ & $32(62.7)$ & $6(11.8)$ & $51(8.7)$ \\
\hline Total & $145(24.8)$ & $379(64.9)$ & $60(10.3)$ & $584(100.0)$ \\
\hline
\end{tabular}


Trend Analysis for Clinical Screening with and Without Instructions from Physician Office

\begin{tabular}{|c|c|c|c|c|}
\hline \multirow{2}{*}{ Year } & \multicolumn{3}{|c|}{ Clinical Screening-Physician Office } & \multirow{2}{*}{ Total, N (\%) } \\
\cline { 2 - 4 } & Good, N (\%) & Fair, N (\%) & Poor, N (\%) & \\
\hline 2003 & $437(67.2)$ & $171(26.3)$ & $42(6.5)$ & $650(10.0)$ \\
\hline 2004 & $519(70.0)$ & $174(23.5)$ & $48(6.5)$ & $741(11.4)$ \\
\hline 2005 & $544(64.3)$ & $242(28.6)$ & $60(7.1)$ & $846(13.1)$ \\
\hline 2006 & $436(60.0)$ & $223(30.7)$ & $68(9.4)$ & $727(11.2)$ \\
\hline 2007 & $490(68.3)$ & $166(23.2)$ & $61(8.5)$ & $717(11.1)$ \\
\hline 2008 & $449(61.8)$ & $189(26.0)$ & $88(12.1)$ & $726(11.2)$ \\
\hline 2009 & $505(70.5)$ & $152(21.2)$ & $59(8.2)$ & $716(11.1)$ \\
\hline 2010 & $302(67.4)$ & $94(21.0)$ & $5211.6)$ & $448(6.9)$ \\
\hline 2011 & $337(69.1)$ & $109(22.3)$ & $42(8.6)$ & $488(7.5)$ \\
\hline 2012 & & & & \\
\hline 2013 & $157(68.9)$ & $59(25.9)$ & $12(5.3)$ & $228(3.5)$ \\
\hline 2014 & $125(67.2)$ & $47(25.3)$ & $14(7.5)$ & $186(2.9)$ \\
\hline Total & $4301(66.4)$ & $1626(25.1)$ & $546(8.4)$ & $6473(100.0)$ \\
\hline
\end{tabular}

Trend Analysis for Clinical Screening Based on Instructions Received from Health Department

\begin{tabular}{|c|c|c|c|c|}
\hline \multirow{2}{*}{ Year } & \multicolumn{2}{|c|}{ Clinical Screening-Health Department } & \multirow{2}{*}{ Total (\%) } \\
\cline { 2 - 4 } & Good, N (\%) & Fair, N (\%) & Poor, N (\%) & \\
\hline 2003 & $16(47.1)$ & $15(44.1)$ & $3(8.8)$ & $34(9.6)$ \\
\hline 2004 & $19(57.6)$ & $12(36.4)$ & $2(6.1)$ & $33(9.3)$ \\
\hline 2005 & $27(58.7)$ & $13(28.3)$ & $6(13.0)$ & $46(13.0)$ \\
\hline 2006 & $20(51.3)$ & $11(28.2)$ & $8(20.5)$ & $39(11.0)$ \\
\hline 2007 & $18(56.2)$ & $12(37.5)$ & $2(6.2)$ & $32(9.0)$ \\
\hline 2008 & $30(65.2)$ & $8(17.4)$ & $8(17.4)$ & $46(13.0)$ \\
\hline 2009 & $13(44.8)$ & $11(37.9)$ & $5(17.2)$ & $29(8.2)$ \\
\hline 2010 & $13(52.0)$ & $11(44.0)$ & $2(2.4)$ & $25(7.0)$ \\
\hline 2011 & $12(48.0)$ & $11(44.0)$ & $2(8.0)$ & $25(7.0)$ \\
\hline 2012 & & & & $2(11.1)$ \\
\hline 2013 & $9(50.0)$ & $7(38.9)$ & $2(7.1)$ & $28(7.9)$ \\
\hline 2014 & $20(71.4)$ & $6(21.4)$ & $2(1)$ & $355(100.0)$ \\
\hline Total & $197(55.5)$ & $117(33.0)$ & $41(1.5)$ & \\
\hline
\end{tabular}


Trend Analysis for Clinical Screening with and without Instructions from Health Department

\begin{tabular}{|c|c|c|c|c|}
\hline \multirow{2}{*}{ Year } & \multicolumn{3}{|c|}{ Clinical Screening-Health Department } & \multirow{2}{*}{ Total, N (\%) } \\
\cline { 2 - 4 } & Good, N (\%) & Fair, N (\%) & Poor, N (\%) & \\
\hline 2003 & $423(65.8)$ & $178(27.7)$ & $42(6.5)$ & $643(10.3)$ \\
\hline 2004 & $485(69.1)$ & $171(24.4)$ & $46(6.6)$ & $702(11.2)$ \\
\hline 2005 & $520(64.5)$ & $227(28.2)$ & $59(7.3)$ & $806(12.9)$ \\
\hline 2006 & $407(58.9)$ & $217(31.4)$ & $67(9.7)$ & $691(11.1)$ \\
\hline 2007 & $478(67.5)$ & $171(24.2)$ & $59(8.3)$ & $708(11.3)$ \\
\hline 2008 & $453(62.8)$ & $182(25.2)$ & $86(11.9)$ & $721(11.5)$ \\
\hline 2009 & $488(69.8)$ & $152(21.7)$ & $59(8.4)$ & $699(11.2)$ \\
\hline 2010 & $289(67.2)$ & $93(21.6)$ & $48(11.2)$ & $430(6.9)$ \\
\hline 2011 & $315(68.5)$ & $106(23.0)$ & $39(8.5)$ & $460(7.4)$ \\
\hline 2012 & & & & \\
\hline 2013 & $148(67.0)$ & $61(27.6)$ & $12(5.4)$ & $221(3.5)$ \\
\hline 2014 & $113(69.3)$ & $40(24.5)$ & $10(6.1)$ & $163(2.6)$ \\
\hline Total & $4,119(66.0)$ & $1,598(25.6)$ & $527(8.4)$ & $6,244(100.0)$ \\
\hline
\end{tabular}

Trend Analysis for Clinical Screening Based on Instructions Received from Other Provider Sources

\begin{tabular}{|c|c|c|c|c|}
\hline \multirow{2}{*}{ Year } & \multicolumn{3}{|c|}{ Clinical Screening-Other Providers } & \multirow{2}{*}{ Total, N (\%) } \\
\cline { 2 - 4 } & Good, N (\%) & Fair, N (\%) & Poor, N (\%) & \\
\hline 2003 & $54(62.8)$ & $20(23.3)$ & $12(14.0)$ & $86(10.6)$ \\
\hline 2004 & $72(69.2)$ & $24(23.1)$ & $8(7.7)$ & $104(12.8)$ \\
\hline 2005 & $64(61.0)$ & $27(25.7)$ & $14(13.3)$ & $105(12.9)$ \\
\hline 2006 & $55(65.5)$ & $15(17.9)$ & $14(16.7)$ & $84(10.3)$ \\
\hline 2007 & $42(60.0)$ & $16(22.9)$ & $12(17.1)$ & $70(8.6)$ \\
\hline 2008 & $33(42.9)$ & $19(24.7)$ & $25(32.5)$ & $77(9.4)$ \\
\hline 2009 & $43(63.2)$ & $19(27.9)$ & $6(8.8)$ & $68(8.3)$ \\
\hline 2010 & $39(60.9)$ & $20(31.2)$ & $5(7.8)$ & $64(7.9)$ \\
\hline 2011 & $40(58.0)$ & $23(33.3)$ & $6(8.7)$ & $69(8.5)$ \\
\hline 2012 & & & & \\
\hline 2013 & $24(60.0)$ & $13(32.5)$ & $3(7.5)$ & $40(4.9)$ \\
\hline 2014 & $35(72.9)$ & $11(22.9)$ & $2(4.2)$ & $48(5.9)$ \\
\hline Total & $501(61.5)$ & $207(25.4)$ & $107(13.1)$ & $815(100.0)$ \\
\hline
\end{tabular}


Trend Analysis for Clinical Screening with and without Instructions from Other Provider Sources

\begin{tabular}{|c|c|c|c|c|}
\hline \multirow{2}{*}{ Year } & \multicolumn{2}{|c|}{ Clinical Screening-Other Providers } & \multirow{2}{*}{ Total, N (\%) } \\
\cline { 2 - 4 } & Good, N (\%) & Fair, N (\%) & Poor, N (\%) & \\
\hline 2003 & $461(66.3)$ & $183(26.3)$ & $51(7.3)$ & $695(10.4)$ \\
\hline 2004 & $538(69.6)$ & $183(23.7)$ & $52(6.7)$ & $773(11.5)$ \\
\hline 2005 & $557(64.4)$ & $241(27.9)$ & $67(7.7)$ & $865(12.9)$ \\
\hline 2006 & $442(60.1)$ & $221(30.0)$ & $73(9.9)$ & $736(11.0)$ \\
\hline 2007 & $502(67.3)$ & $175(23.5)$ & $69(9.2)$ & $746(11.1)$ \\
\hline 2008 & $456(60.6)$ & $193(25.7)$ & $103(13.7)$ & $752(11.2)$ \\
\hline 2009 & $518(70.2)$ & $160(21.7)$ & $60(8.1)$ & $738(11.0)$ \\
\hline 2010 & $315(67.1)$ & $102(21.7)$ & $52(! 1.1)$ & $469(7.0)$ \\
\hline 2011 & $343(68.1)$ & $118(23.4)$ & $43(8.5)$ & $504(7.5)$ \\
\hline 2012 & & & & \\
\hline 2013 & $163(67.1)$ & $67(27.6)$ & $13(5.3)$ & $243(3.6)$ \\
\hline 2014 & $128(69.9)$ & $45(24.6)$ & $10(5.5)$ & $183(2.7)$ \\
\hline Total & $4,423(66.0)$ & $1,688(25.2)$ & $593(8.8)$ & $6,704(100.0)$ \\
\hline
\end{tabular}

\section{Trend Analysis for Parental Assessment Vs Clinical Screening-Dental Office}

\begin{tabular}{|c|c|c|c|c|}
\hline \multirow{2}{*}{ Year } & \multicolumn{3}{|c|}{ Parental Vs Clinical Screening } & \multirow{2}{*}{ Total, N (\%) } \\
\cline { 2 - 4 } & Correct, N (\%) & Wrong, N (\%) & Way, N (\%) & \\
\hline 2003 & $2,178(69.94)$ & $834(26.78)$ & $102(3.28)$ & $3,114(11.05)$ \\
\hline 2004 & $1,857(67.87)$ & $773(28.25)$ & $106(3.87)$ & $2,736(9.71)$ \\
\hline 2005 & $2,055(66.12)$ & $918(29.54)$ & $135(4.34)$ & $3,108(11.03)$ \\
\hline 2006 & $1,850(64.26)$ & $882(30.64)$ & $147(5.11)$ & $2,879(10.22)$ \\
\hline 2007 & $1,603(59.60)$ & $960(35.75)$ & $122(4.54)$ & $2,685(9.53)$ \\
\hline 2008 & $1,606(60.40)$ & $931(35.01)$ & $122(4.59)$ & $2,659(9.44)$ \\
\hline 2009 & $1,957(65.41)$ & $946(31.62)$ & $89(2.97)$ & $2,992(10.62)$ \\
\hline 2010 & $1,535(62.76)$ & $843(34.46)$ & $68(2.78)$ & $2,446(8.68)$ \\
\hline 2011 & $1,691(64.00)$ & $844(31.95)$ & $107(4.05)$ & $2,642(9.38)$ \\
\hline 2012 & & & & \\
\hline 2013 & $1,086(65.03)$ & $542(32.46)$ & $42(2.51)$ & $1,670(5.93)$ \\
\hline 2014 & $843(67.82)$ & $374(30.09)$ & $26(2.09)$ & $1,243(4.41)$ \\
\hline Total & $18,261(64.82)$ & $8,847(31.40)$ & $1,066(3.78)$ & $28,174(100.00)$ \\
\hline
\end{tabular}




\section{Trend Analysis for Parental Assessment Vs Clinical Screening- Health Department}

\begin{tabular}{|c|c|c|c|c|}
\hline \multirow{2}{*}{ Year } & \multicolumn{2}{|c|}{ Parental Vs Clinical Screening } & \multirow{2}{*}{ Total, N (\%) } \\
\cline { 2 - 4 } & Correct, N(\%) & Wrong, N (\%) & Way, N (\%) & \\
\hline 2003 & $386(60.03)$ & $226(35.15)$ & $31(4.82)$ & $643(10.30)$ \\
\hline 2004 & $453(64.53)$ & $211(30.06)$ & $38(5.41)$ & $702(11.24)$ \\
\hline 2005 & $486(60.30)$ & $260(32.26)$ & $60(7.44)$ & $806(12.91)$ \\
\hline 2006 & $400(57.89)$ & $240(34.73)$ & $51(7.38)$ & $691(11.07)$ \\
\hline 2007 & $378(53.39)$ & $296(41.81)$ & $34(4.80)$ & $708(11.34)$ \\
\hline 2008 & $395(54.79)$ & $285(39.53)$ & $41(5.69)$ & $721(11.55)$ \\
\hline 2009 & $413(59.08)$ & $255(36.48)$ & $31(4.43)$ & $699(11.19)$ \\
\hline 2010 & $230(53.49)$ & $179(41.63)$ & $21(4.88)$ & $430(6.89)$ \\
\hline 2011 & $274(59.57)$ & $164(35.65)$ & $22(4.78)$ & $460(7.37)$ \\
\hline 2012 & & & & \\
\hline 2013 & $127(57.47)$ & $85(38.46)$ & $9(4.07)$ & $221(3.54)$ \\
\hline 2014 & $90(55.21)$ & $69(42.33)$ & $04(2.45)$ & $163(2.61)$ \\
\hline Total & $3632(58.17)$ & $2270(36.35)$ & $342(5.48)$ & $6244(100.00)$ \\
\hline
\end{tabular}

Trend Analysis for Parental Assessment Vs Clinical Screening-Physician Office

\begin{tabular}{|c|c|c|c|c|}
\hline \multirow{2}{*}{ Year } & \multicolumn{2}{|c|}{ Parental Vs Clinical Screening } & \multirow{2}{*}{ Total, N (\%) } \\
\cline { 2 - 4 } & Correct, N (\%) & Wrong, N (\%) & Way, N (\%) & \\
\hline 2003 & $387(59.54)$ & $230(35.38)$ & $33(5.08)$ & $650(10.04)$ \\
\hline 2004 & $474(63.97)$ & $228(30.77)$ & $39(5.26)$ & $741(11.45)$ \\
\hline 2005 & $519(61.35)$ & $270(31.91)$ & $57(6.74)$ & $846(13.07)$ \\
\hline 2006 & $428(58.87)$ & $248(34.11)$ & $51(7.02)$ & $727(11.23)$ \\
\hline 2007 & $379(52.86)$ & $303(42.26)$ & $35(4.88)$ & $717(11.08)$ \\
\hline 2008 & $403(55.51)$ & $278(38.29)$ & $45(6.20)$ & $726(11.22)$ \\
\hline 2009 & $428(59.78)$ & $255(35.61)$ & $33(4.61)$ & $716(11.06)$ \\
\hline 2010 & $235(52.46)$ & $192(42.86)$ & $21(4.69)$ & $448(6.92)$ \\
\hline 2011 & $286(58.61)$ & $179(36.68)$ & $23(4.71)$ & $488(7.54)$ \\
\hline 2012 & & & & \\
\hline 2013 & $136(59.65)$ & $83(36.40)$ & $9(3.95)$ & $228(3.52)$ \\
\hline 2014 & $100(53.75)$ & $82(44.09)$ & $04(2.15)$ & $186(2.87)$ \\
\hline Total & $3775(58.32)$ & $2348(36.27)$ & $350(5.41)$ & $6473(100.00)$ \\
\hline
\end{tabular}


Trend Analysis for Parental Assessment Vs Clinical Screening-Other Providers

\begin{tabular}{|c|c|c|c|c|}
\hline \multirow{2}{*}{ Year } & \multicolumn{2}{|c|}{ Parental Vs Clinical Screening } & \multirow{2}{*}{ Total, N (\%) } \\
\cline { 2 - 4 } & Correct, N (\%) & Wrong, N (\%) & Way, N (\%) & \\
\hline 2003 & $419(60.29)$ & $242(34.82)$ & $34(4.89)$ & $695(10.37)$ \\
\hline 2004 & $500(64.68)$ & $231(29.88)$ & $42(5.43)$ & $773(11.53)$ \\
\hline 2005 & $527(60.92)$ & $273(31.56)$ & $65(7.51)$ & $865(12.90)$ \\
\hline 2006 & $429(58.29)$ & $249(33.83)$ & $58(7.88)$ & $736(10.98)$ \\
\hline 2007 & $398(53.35)$ & $305(40.88)$ & $43(5.76)$ & $746(11.13)$ \\
\hline 2008 & $415(55.19)$ & $287(38.16)$ & $50(6.65)$ & $752(11.22)$ \\
\hline 2009 & $431(58.40)$ & $273(36.99)$ & $34(4.61)$ & $738(11.01)$ \\
\hline 2010 & $246(52.45)$ & $200(42.64)$ & $23(4.90)$ & $469(7.00)$ \\
\hline 2011 & $297(58.93)$ & $181(35.91)$ & $26(5.16)$ & $504(7.52)$ \\
\hline 2012 & & & & \\
\hline 2013 & $142(58.44)$ & $92(37.86)$ & $09(3.70)$ & $243(3.62)$ \\
\hline 2014 & $105(57.38)$ & $74(40.44)$ & $04(2.19)$ & $183(2.73)$ \\
\hline Total & $3,909(58.31)$ & $2,407(35.90)$ & $388(5.79)$ & $6,704(100.00)$ \\
\hline
\end{tabular}

Trend Analysis for Parental Assessment Vs Clinical Screening-No Instruction

\section{Received}

\begin{tabular}{|c|c|c|c|c|}
\hline \multirow{2}{*}{ Year } & \multicolumn{2}{|c|}{ Parental Vs Clinical Screening } & \multirow{2}{*}{ Total, N (\%) } \\
\cline { 2 - 4 } & Correct, N (\%) & Wrong, N (\%) & Way, N (\%) & \\
\hline 2003 & $366(60.10)$ & $214(35.14)$ & $29(4.76)$ & $609(10.34)$ \\
\hline 2004 & $430(64.28)$ & $203(30.34)$ & $36(5.28)$ & $669(11.36)$ \\
\hline 2005 & $462(60.79)$ & $243(31.97)$ & $55(7.24)$ & $760(12.91)$ \\
\hline 2006 & $377(57.82)$ & $228(34.97)$ & $47(7.21)$ & $652(11.07)$ \\
\hline 2007 & $363(53.70)$ & $279(41.27)$ & $34(5.03)$ & $676(11.48)$ \\
\hline 2008 & $379(56.15)$ & $256(37.93)$ & $40(5.93)$ & $675(11.46)$ \\
\hline 2009 & $402(60.00)$ & $238(35.52)$ & $30(4.48)$ & $670(11.38)$ \\
\hline 2010 & $215(53.09)$ & $171(42.22)$ & $19(4.69)$ & $405(6.88)$ \\
\hline 2011 & $262(60.23)$ & $152(34.94)$ & $21(4.83)$ & $435(7.39)$ \\
\hline 2012 & & & & \\
\hline 2013 & $122(60.10)$ & $74(36.45)$ & $7(3.45)$ & $203(3.45)$ \\
\hline 2014 & $73(54.07)$ & $60(44.44)$ & $2(1.48)$ & $135(2.29)$ \\
\hline Total & $3,451(58.60)$ & $2,118(35.97)$ & $320(5.43)$ & $5,889(100.00)$ \\
\hline
\end{tabular}


Table 26: Trend Analysis for Cavities, Insurance and In-office Visits over the Past 11 Years

\begin{tabular}{|c|c|c|c|}
\hline \multirow{2}{*}{ Year } & \multicolumn{3}{|c|}{ Cavities } \\
\cline { 2 - 4 } & Cavities, N (\%) & Insurance, $\mathbf{N}(\%)$ & $\begin{array}{c}\text { In-office Visits, } N \\
(\%)\end{array}$ \\
\hline 2003 & $1,111(31.0)$ & $3,228(90.1)$ & $3,337(93.16)$ \\
\hline 2004 & $1,096(33.3)$ & $2,518(76.6)$ & $2,944(89.54)$ \\
\hline 2005 & $1,221(33.0)$ & $2,822(76.4)$ & $3,340(90.37)$ \\
\hline 2006 & $1,140(32.9)$ & $2,687(77.7)$ & $3,148(90.98)$ \\
\hline 2007 & $943(29.0)$ & $2,524(77.7)$ & $3,038(93.56)$ \\
\hline 2008 & $1,102(34.5)$ & $2,545(79.7)$ & $2,971(92.99)$ \\
\hline 2009 & $932(26.6)$ & $2,816(80.2)$ & $3,345(95.30)$ \\
\hline 2010 & $809(27.9)$ & $2,595(89.5)$ & $2,728(94.13)$ \\
\hline 2011 & $838(26.6)$ & $2,726(86.7)$ & $2,968(94.37)$ \\
\hline 2012 & & & \\
\hline 2013 & $483(24.8)$ & $1,777(91.1)$ & $1,887(96.72)$ \\
\hline 2014 & $352(24.1)$ & $1,275(87.3)$ & $1,410(96.58)$ \\
\hline Total & $10,027(30.0)$ & $27,513(82.3)$ & $31,116(93.1)$ \\
\hline
\end{tabular}




\section{CURRICULUM VITAE}

NAME: Sireesha Kodali

ADDRESS: 789 Eastern Parkway Apt\#2

Louisville, KY 40217

DOB: Malakapalli, India- May 22, 1987

EDUCATION \& TRAINING:

BDS, Bachelor of Dental Surgery

NTR University of Health Sciences, Vishnu Dental College

2005-2010

MHA, Healthcare Administration

Western Kentucky University

2012-2013

DMD, Doctor of Dental Medicine

University of Louisville School of Dentistry, Louisville

2014-2016

MSOB, Oral Biology

University of Louisville School of Dentistry, Louisville

2015-2016

\section{AWARDS:}

Zuhier \& Sophia Award for Academic Scholarship

Western Kentucky University, Bowling Green, KY

2013

\section{PUBLICATIONS:}

$>$ Survival of Preterm Singleton Deliveries: A Population-Based Retrospective Study, Advances in Epidemiology, vol. 2015, Article ID 858274, 6 pages, 2015. doi:10.1155/2015/858274

$>$ A Review of Occupational Health Implications of Exposure and Risk Management of Carbon Nanotubes and Carbon Nanofibers, International Journal of Environmental Science and Development, Vol.7, No.11, November 2016 


\section{PRESENTATIONS:}

$>$ A review of literature on nanoparticles: symposium at University of Cincinnatti

$>$ Influence of in-office hygiene instructions on parental assessment of their child's dental condition: Research Louisville, 2014 